\title{
A Study of Signal Generation and Charge Collection in a-Si:H Diodes for Radiation Imaging
}

\author{
Ichiro Fujieda \\ Ph.D. Thesis \\ Physics Division \\ Lawrence Berkeley Laboratory \\ University of California \\ Berkeley, CA 94720
}

October 1990

The U.S. Deparment of Energy has the right to use this thesis for any purpose whatsoever including the right to reproduce all or any pan thereof.

This work was supported by the U.S. Department of Energy under

Contract No. DE-AC03-76SF00098. 


\title{
A Study of Signal Generation and Charge Collection in a-Si:H Diodes for Radiation Imaging
}

\author{
Ichiro Fujieda
}

\begin{abstract}
Hydrogenated amorphous silicon (a-Si:H) has been investigated as a material for radiation detectors. Its high radiatien resistivity and large-area capability are the expected advantages of this material together with its ability to provide a front-end readout electronics in the vicinity of the sensor element. Electrons and holes created by inc'sming charged particles, $\mathrm{X}$-rays, $\gamma$ rays, are drifted by the electric field inside a-Si:H diodes and this carrier movement induces signal charges on electrodes. Charge collection and signal generation process are analyzed in terms of carrier mobilities, lifetimes and electric field. Charge collection in thick a-Si:H diodes is often limited by deep-level trapping of carriers during transit and a finite charge integration time required for single particle counting in some applications and sometimes by volume recombination of carriers for detecting heavily-ionizing particles such as $\alpha$ particles. The charge collection process is also strongly affected by the non-uniform electric field profiles in a-Si:H-diodes caused by the fixed space charges inside the material under reverse-bias. Signal generation due to a weak light pulse irradiating eaclt end of a thick diode is measured as a function of a reversebias and it gives a valuable information about the fixed space charges. Field profiles can be manipulated by either doping, electrode geometry, or combination of both to improve the charge collection process. One can apply a higher reverse-bias on a diode with an
\end{abstract}


equivalent thickness by providing buffer layers at each end of the diode and thus suppressing soft breakdown phenomena. $\mathbf{X}$-ray detection with a good sensitivity is demonstrated by an a-Si:H photodiode coupled to an evaporated Csi scintillator. The scintillation quality of evaporated CsI layers can be made almost identical to its single crystal counterpart. Fields of a.Si:H radiation detector application include high energy physics, medical imaging, materials science and life science.

Thesis commitee: Selig N. Kaplan (chair)

Victor Perez-Mendez

Chenming Hu 


\section{A Study of Signal Generation and Charge Collection in a-Si:H Diodes for Radiation Imaging}

Copyright c 1990

Ichiro Fujieda

The United States Department of Energy has the right to use this thesis for any purpose whatsoever including the right to reproduce alt or any part thereot.

The Goverment reserves for itself and others acting on its behalf a royalty free, nonexclusive, irrevocable, world-wide license for govermental purposes to publish, distribute, translate, duplicate, exhibit, and perform any such data opyrighted by the contractor. 


\section{Acknowledgements}

I would like to express my appreciation to Professors Victor Perez-Mendez, Selig N. Kaplan and Chenming Hu for their warm guidance and great ideas and suggestions to complete this thesis. I would like to thank to the members of our group, Dr. J. Drewery, my fellow graduate students, Gyuseong Cho, Tao Jing, Shafi Qureshi, Daniel Wildermuth, Kaarlo Heiskaiten for experimental assistance and many disccusions we had during the course of my studies. My appreciation is also extended to Drs. R.A. Street, R.L. Weisfield and C.C. Tsai of Xerox Palo Alto Research Center and Dr. J. Xi of Glasstech Solar Inc. for helpful discussions and technical supports such as making a-Si:H samples. I am also grateful to Dr. D. Johnson of Varian Corp. and Dr. B. Utts of Engelhard Corp. for their valuable comments about the CsI evaporation process and T. Gee and Dr. R.D. Giauque of Lawrence Berkeley Laboratory for phosphor preparation and the XRF analysis. I am also indebted to Dr. Y. Tsukuda of Hitachi Metal Lid. for supplying us the $\mathrm{GdO}_{2} \mathrm{~S}_{2}$ scintillator.

Finally, I would like to thank my wife, Miho and my parents who continue their supports in every aspect of my life. 


\section{Table of Contents}

Chapter I Introduction 1

1-1. Production methods and basic properties of a-Si:H

1-1-1. Production methods

1-1-2. Disordered structure

1-1-3. Density of states and carrier transport

1-1-4. Doping

1-2. a-Si:H radiation detectors

6

1-2-1. Historical background

1-2-2. Detection principles

References

9

11

Chapter 2. Signal generation in a-Si:H radiation detectors

2-1. Introduction

13

2-2-1. Instant charge creation at one end of a diode

2-2-2. Instant charge creation uniformly through a diode

2-3 Measurement

19

2-3-1. Signal size dependence on a bias

2-3-2. Signal size dependence on peaking time

2-3-3. Nd* dependence on time after bias application

2-4. Discussion

2-5. Conclusion

Appendix Time of Flight analysis

29

A 1. Time of Flight Basics

A2. DC bias TOF

References

$\begin{array}{ll}\text { Chapter 3. Field profile tailoring } & 36\end{array}$

3-1. Introduction 36

3-2. Tailoring by doping 38

3-2-1. Field profile calculation for multilayer structures

3-2-2. Buried $\mathrm{p}-\mathrm{i}-\mathrm{n}$ diode

3-2-2-1. Fabricatrion

3-2-2-2. Noise and I-V measurements

3-2-2-3. Signal generation from $\alpha$ particles

Theory

Measurements

Analysis

3-2-3. $p-\mathrm{i}-n$ diode with thick $\mathrm{p}$ layer

3-2-3-1. Fabrication

3-2-3-2. Noise and I-V measurements

3-2-3-3. Discussions of relative merits

3-2-4. Breakdown and ionized defect density

3-2-5. $\mathrm{p}-\mathrm{i}-\mathrm{n}$ diode with buried $\mathrm{p}$ layer 
3-3. Tailoring by electrode shapes

3-3-1. Field profile calculation for the cylindrical configuration

3-3-2. Microstrip electrode

3-3-3. Fabrication and measurements of microstrip electrode

3-4. Conclusion

61

References

62

Chapter 4. Scintillation light detection 64

4-1. Introduction 64

4-2. CsI evaporation 68

4-3. X-ray test setup and Csi signal yield measurement 69

4-3-1. X-ray source and test setup description

4-3-2. X-ray source calibration and CsI signal yield measurement 4-3-3. Error analysis

4-4. Light yield optimization

4-4-1. Light yield of CsI(Na)

4-4-2. Light yield of CsI(Tl)

4-4-3. Tl concentration in an evaporated CsI(Tl)

4-5. Speed of $\mathrm{CsI(TI)}$ and $\mathrm{Gd}_{2} \mathrm{O}_{2} \mathrm{~S}$ screens

4-5-1. Theoretical analysis

4-5-2. Measurement

4-5-3. Analysis

4-6. Spatial resolution measurement

76

4-7. Radiation damage to an evaporated CsI(Tl)

88

4-8. $X$-ray and $\beta$ particle detection with CsI coupled to a photodiode

96

4-8-1. X-ray detection

4-8-2. $\beta$ particle detection

4-9. Monolithic X.ray sensor

97

4-10. Conclusion

101

Appendix Summary of various evaporation nuns 105

References

107

Chapter 5. Applications of a-Si:H radiation detectors 109

5-1. Position sensitivity 109

5-2. High energy physics applications 111

5-3. Medical imaging

5-3-1. Flux imaging devices

5-3-2. Single photon imaging devices

5-4. Materiai scierice and life science applications

5-5, Conclusion

References 


\section{Chapter IIntroduction}

Hydrogenated amorphous silicon (a-Si:H) is a relatively new semiconductor material. Since as first demonstrated by Spear and LeComber in 1976 that it could be doped to form p or n-type material, it has been widely used in industry as the material for solar cells, facsimile heads and liquid crystal display drivers. In this chapter, some production methods and basic propurties of a-Si:H are reviewed first. An extensive review of a-Si:H preparation, structure, optical and electronic properties and device applications (except for radiation detection) can be found in ref.[1]. The historical background of its use as a radiation detector material and some detection principles of these detectors are also described in this chapter.

\section{1-1. Production methods and basic properties of a-Si:H}

\section{1-1-1. Production methods}

A-Si:H layers are deposited on heated substrates $\left(\leq 250^{\circ} \mathrm{C}\right)$ most commonly by the Glow-Discharge decomposition of $\mathrm{SiH}_{4}$ gas by $13.54 \mathrm{MHz} \mathrm{RF}$ power. This method is called Plasma Enhanced Chemical Vapor Deposition (PECVD). Conditions such as substrate temperature, RF power, flow rate and pressure of $\mathrm{SiH}_{4}$ gas all play important roles in depositing a-Si: $\mathrm{H}$ layers and these are determined largely by lengthy trial-error experiments.

Doping a-Si:H layers can be achieved by adding dopant gases such as $\mathrm{PH}_{3}$ and $\mathrm{B}_{2} \mathrm{H}_{6}$ to $\mathrm{SiH}_{4}$. Multi-chamber systems such as shown in Fig.1-1 are routinely used to avoid crosscontamination of gases at $\mathrm{p}-\mathrm{i}-\mathrm{n}$ a-Si:H solar cell production facilities.

Radicals ( $\left.\mathrm{SiH}_{n}, 0 \leq n \leq 3\right)$ created by the RF discharge either stick to or difflise on the growing layer and eventually form a $\mathrm{Si}-\mathrm{Si}$ network by chemical reactions assisted by the heat provided from the substrate. If the sticking process dominates over the radical propagation on the substrate surface, the deposition is called Physical Vapor Deposition (PVD) and if the radical propagation dominates, it is called Chemical Vapor Deposition 
(CVD). PVD occurs at high deposition rates with high RF power and the resultant Si network is three dimensional isiand-like, that is morphologically inhomogeneous. Electronic properties of these layers are poor and cannot be used for many device applications. If the RF power is kept low and all other parameters are optimized, closelypacked morphologically uniform arnorphous Si network results by CVD and these layers are often called "device-quality" [1].

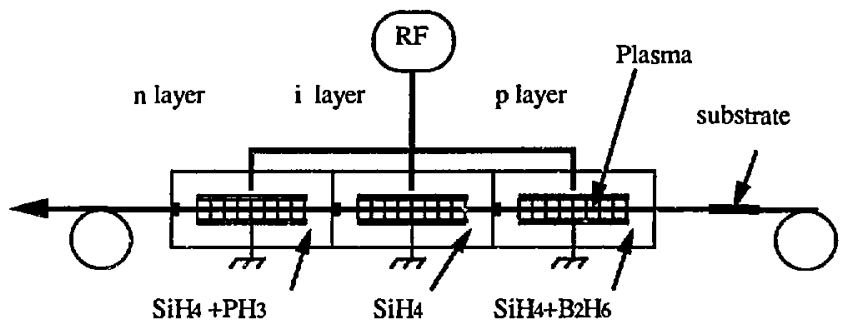

Fig.1-1 Multi-chamber system for p-i-n a-Si:H deposition

The deposition rate of the standard Glow Discharge (GD) method of producing "device quality a-Si:H" is normally about $1 \mu \mathrm{m} / \mathrm{hr}$ and is the limiting factor for throughput of solar cell production. Efforts have been focused on increasing the deposition rate without degrading the properties of a-Si:H. Variations of the GD method include the use of higher RF frequency (VHF-CVD method) [2,3], addition of hydrogen and fluorine radicals (HRCVD) [4], and the use of magnetic field (Controlled Plasma Magnetron method (CPM)) [5]. Higher deposition rates are also obtained by the use of disilane $\mathrm{Si}_{2} \mathrm{H}_{6}$ instead of silane [6] or by the plasma CVD induced by Electron Cyclotron Resonance (ECR) [7]. Instead of RF plasma, photo-excited $\mathrm{Hg}$ atoms can also decompose silane gas to produce aSi:H layer (photo-CVD) [8]. Some reported characteristics of a-Si:H layers deposited by these various techniques are summarized in the Table 1-1. The listed defect densities are measured by different characterization techniques. Since different characterization 
techniques give different aspects of the "defect density", the table below should convey only a rough picture. VHF and CPM are promising for fast deposition of good quality aSi:H layeis. As for the thickness comparison, many techniques are meant for solar cell and photo-sensor production, hence the thickness limitation has not been pushed beyond $1 \mu \mathrm{m}$. It has been already demonstrated that thick layers can be deposited successfully by GD and VHF.

Table 1-1. a-Si:H preparation techniques

\begin{tabular}{|c|c|c|c|c|}
\hline technique & defect density & deposition rate & thickness & ref. \\
\hline GD (silane) & $2 \times 10^{+15} \mathrm{~cm}^{-3}$ & $1 \mu \mathrm{m} / \mathrm{hr}$ & $\leq 50 \mu \mathrm{m}$ & 1 \\
\hline VHF-CVD & $2-6 \times 10^{+15} \mathrm{~cm}^{-3}$ & $2-6 \mu \mathrm{m} / \mathrm{hr}$ & $200 \mu \mathrm{m}$ & 2 \\
\hline CPM-CVD & $7 \times 10^{+14} \mathrm{~cm}^{-3}$ & $2-3 \mu \mathrm{m} / \mathrm{hr}$ & $1 \mu \mathrm{m}$ & 5 \\
\hline ECR-CVD & $2-3 \times 10^{+16} \mathrm{~cm}^{-3}$ & $8 \mu \mathrm{m} / \mathrm{hr}$ & $?$ & 7 \\
\hline HR-CVD & as good as GD & $1-2 \mu \mathrm{m} / \mathrm{hr}$ & $\leq 10 \mu \mathrm{m}$ & 4 \\
\hline Photo-CVD & as good as GD & $\leq 1 \mu \mathrm{m} / \mathrm{hr}$ & $1 \mu \mathrm{m}$ & 8 \\
\hline GD (disilane) & higher than GD & $2-3 \mu \mathrm{m} / \mathrm{hr}$ & $1 \mu \mathrm{m}$ & 6 \\
\hline
\end{tabular}

\section{1-1-2. Disordered structure}

A schematic view of the a-Si:H network is shown in Fig.1-2. Si atoms have four bonds to form a Si-Si network but not all of them are necessarily used. The unused bonds are called "dangling bonds" and represent an undesirable feature as will be discussed later. The number of dangling bonds are greatly reduced by inclusion of $\mathrm{H}$ atoms which "terminate" the unpaired electrons. The interatomic distance is not constant as is the case of crystalline Si. The lack of long-range order while retaining a short-range order in the $\mathrm{Si-Si}$ network results in a peculiar density of states which affects carrier transport properties. These properties will be discussed further below. 


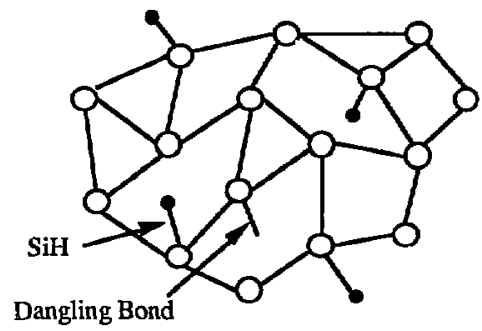

Fig.1-2 Schematic view of a-Si:H network

\section{1-1-3. Density of states and carrier transport}

Many characterization techniques have lead us to the understanding of the density of states of "intrinsic" a-Si:H as shown schematically in Fig.1-3. There are two major differences from the intrinsic crystalline silicon case, which affect carrier transport properties.

First, the dangling bonds create mid-gap states. The "intrinsic" a-Si:H material is slightly n-type in a sense that the material leaves a small but significant amount of fixed positive charges when these dangling bonds release the unpaired electrons when an electric field is applied. The way that the dangling bonds affect carrier transport properties is twofold. First, the fixed positive space charge causes the electric field to drop down with distance, producing a non-uniform field profile through the material. Secondly, these midgap states act as recombination centers since the thermal release of trapped carriers is less likely than the subsequent trapping of the opposite type carriers.

Second, the edges of the conduction and valence bands become "fuzzy" due to the nonconstant interatomic distance. The resultant "tail states" affects the carrier transport properties. It is now largely accepted that electron transport is described by the "multitrapping model" and hole transport by "hopping" at room temperature [1]. Electrons in the conduction band are e:sily trapped by the tail states and thermally released in a shor time to 
continue their transport. The width of the valence band tail states is larger than that of the conduction band tail states. Hole transport occurs largely in this tail as a succession of tunnelings through the potential barriers between two adjacent trapping ceuters, "hopping."

A.s a resuli of thers mid-gap states and the tai! states, the carrier transport properties, such as mobility and lifetime of a-Si:H are inferior w those of crystalline silicon.

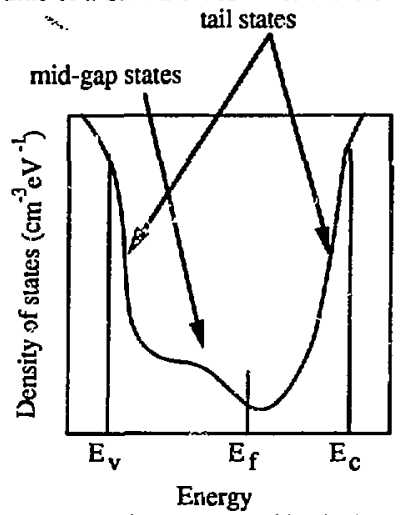

Fig.1-3 Density of states of intrinsic a-Si:H

Important material properties of intrinsic a-Si:H are summarized and compared to those of crystalline $\mathrm{Si}$ in the table below.

Table.1-2 Properties of a-Si:H and crystalline Si

\begin{tabular}{|c|c|c|}
\hline properties & $\mathrm{a}-\mathrm{Si}: \mathrm{H}$ & $\mathrm{X}$ tal Si \\
\hline Density & $2.25-2.3 \mathrm{~g} / \mathrm{cc}$ & $2.3 \mathrm{~g} / \mathrm{cc}$ \\
\hline Dielectric const. & 11.8 & 12.0 \\
\hline Resistivity & $10^{+9}-10^{+10} \Omega \mathrm{cm}$ & $<2 \times 10^{+5} \Omega \mathrm{cm}$ \\
\hline Band gap & $1.7-1.9 \mathrm{eV}$ & $1.1 \mathrm{eV}$ \\
\hline Mobility; electron & $1 \mathrm{~cm}^{2} / \mathrm{Vsec}$ & $1350 \mathrm{~cm}^{2}, \mathrm{Vsec}$ \\
\hline hole & $0.005 \mathrm{~cm}^{2} / \mathrm{sec}$ & $480 \mathrm{~cm}^{2} / \mathrm{Vsec}$ \\
\hline
\end{tabular}




\section{1-1-4. Doping}

When a doped a-Si:H material is deposited, the incorporated $\mathrm{P}$ or $\mathbf{B}$ atoms create an impurity band close to the conduction band or the valence band, respectively, togetier with the "doping-induced dangling bonds" in the mid-gap according by the following reactions (Street's model) [9].

$$
\begin{aligned}
& \mathrm{Si}+\mathrm{P} \Rightarrow>\mathrm{Si}_{3}{ }^{-}+\mathrm{P}_{4}{ }^{+} \\
& \mathrm{Si}+\mathrm{B} \Rightarrow \mathrm{Si}_{3}{ }^{+}+\mathrm{B}_{4}{ }^{-}
\end{aligned}
$$

The + or - sign represents the charge of the doping-induced dangling bonds and the subscript is the number of surrounding Si atoms, known as the coordination number. These charged dangling bonds are partially neutralized by the opposite type sarriers so that the resultant material leaves net fixed space charge of opposite sign represented by $\mathrm{P}_{4}{ }^{+}$or $\mathrm{B}_{4}^{-}$when the material is under bias.

\section{1-2. a-Si:H radiation detectors}

\section{1-2-1. Historical background}

a-Si: $\mathbf{H}$ has been investigated as a possible altemative for radiation detector material for high energy physics experiments, medical imaging, material and life science studies. Early efforts to make radiation detectors with a-Si:H were limited to the thickness range of 1-2 $\mu m$. Wei et al. [10] made a 16 element $X$-ray sensor with each element size of $3 \mathrm{~mm} \times 16$ mm. X-ray fluences were successfully detected by this device with a good linearity between the output signal size and the input $X$-ray intensity. When coupled to a phosphor such as $\mathrm{CdWO}_{4}$ and $\mathrm{ZnS}(\mathrm{Ni})$, a larger output was obtained due to the increased interaction efficiency of the individual X-ray photons. Mochiki et al. [11] used a facsimile head to detect $X$-ray fluences and obtained similar results with an improved spatial resolution. Naruse et al. [12] have proposed the concept of the metal/a-Si:H multilayer structure, which looks like a miniature calorimeter as used in high energy physics experiments. 
Recently, Ito et al. fabricated a monolithic type $X$-ray sensor by depositing $2-\mathrm{Si}: \mathrm{H}$ layers directly on a ceramic scintillator [13]. Street et al. obtained $X$-ray images with a 2-D aSi:H photdiode array.coupled to the intensifying screen [14]. All of these groups used 1-2 $\mu \mathrm{m}$ thick a-Si:H layers to detect $\mathrm{X}$-ray fluences. Other groups focused on making thicker a-Si:H layers up to $50 \mu \mathrm{m}$ for single particle counting so that the incoming radiation deposits enough energy in the material to produce a large signal well above the noise $[15,16,17]$.

\section{1-2-2. Detection principles}

Figure $1-4$ illustrates some possible detection schemes for a- $\mathrm{Si}: \mathrm{H}$ radiation detectors. Depending on the kinds of radiations and applications, one of the following detection schemes has advantages over the others.

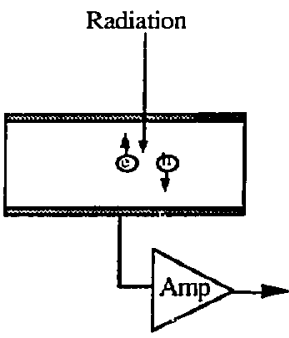

(a)

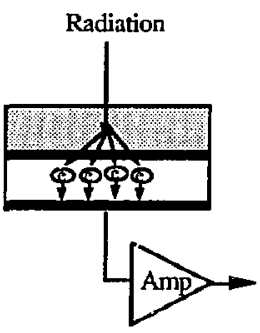

(b)

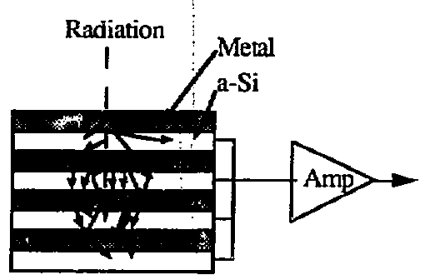

(c)

Fig.1-4. Detection schemes of a-Si:H radiation detectors. (a) Direct detection scheme. (b) Indirect detection scheme. (c) Calorimeter scheme.

In the direct detection scheme as shown in Fig. 1-4(a), charged particles cause ionization events in the thick depleted a-Si:H layer. The average energy required to produce one electron-hole pair, known as the $\mathrm{W}$ value, is about $5 \mathrm{eV}$ [18], almost three times the gap energy of a-Si:H (about $1.8 \mathrm{eV}$ ). This ratio of the $\mathrm{W}$ value to the gap energy seems to be a characteristic of semiconductors [19]. These radiation-induced electron-hole pairs drift in 
the electric field, inducing current in the extemal circuit which is amplified by the external electronics. Readout electronics may be designed to count either individual particles or fluences, depending on the applications of the detectors. The signal generation process in a-Si:H diodes is discussed in Chapter 2. A problem in operating thick a-Si:H diodes and some possible solutions are described in Chapter 3.

In the indirect detection scheme shown in Fig.1-4(b), a phosphor (scintillator) converts the incident radiation (charged particles, $\gamma$ rays or $\mathrm{X}$-ray fluences) into visible light $(\lambda=350-600 \mathrm{~nm})$ which is then absorbed mostly at the surface of the thin $(1-2 \mu \mathrm{m}) \mathrm{a}-\mathrm{Si}: \mathrm{H}$ layer. Electrons and holes are created near the surface and are drifted by the field. This scheme is discussed in detail in Chapter 4.

In the calorimeter scheme as shown in Fig.1-4(c), a succession of alternating metal and a-Si:H layers are stacked. Incident high-energy photons undergo photoelectric, Compton, and pair-production interactions preferentially in the metal layers due to their higher atomic number. The resultant electrons which emerge from the metal converter layers produce electron-hole pairs in the a-Si:H layers.

Since a-Si:H is prepared by decomposition of $\mathrm{SiH}_{4}$ gas in glow discharge on low temperature substrates, large area detectors can be easily fabricated at low cost. The disordered $\mathrm{Si}-\mathrm{Si}$ network makes the material less sensitive to radiation-induced damages. Much of such damage can be removed by subsequent annealing at low temperature [16]. Although its material parameters, such as carrier mobility, life time and defect density are less attractive than those of crystalline silicon, there are various applications in which the advantages of a-Si:H described above outweigh its less attractive material parameters. Some of these expected application fields are described in Chapter 5. 


\section{References}

1. Semiconductors and Semimetals Vol.21 Hydrogenated Amorphous Silicon Part A Preparation and Structure, Part B Optical Properties, Part C Electronic and Transport Properties, Part D Device Applications, Edited by J.I. Pankove, Academic Press Inc., 1984.

2. H. Curtins, W. Wyrsch, and A.V. Shah, "High-Rate Deposition of Amorphous Hydrogenated Silicon: Effect of Plasma Excitation Frequency," Electron. Lett.23(1987)228.

3. S. Oda, J. Noda and M. Matsumura, "Preparation of a-Si:H Films by VHF Plasma CVD," Proc. Mater. Soc.Vol.118(1988) 117.

4. N. Shibata, K. Fukuda, H. Ohtoshi, J. Hanna, S. Oda and I. Shimizu, "Groth of Amorphous Silicon by HR-CVD (Hydrogen Radical Enhanced CVD)," Proc. Mater. Soc.Vol.95(1987) 225.

5. M. Ohnishi, H. Nishiwaki, K. Uchihashi, K. Yoshida, M. Tanaka, K. Ninomiya, M. Noshikuni, N. Nakamura, S. Tsuda, S. Nakano, T. Yazaki and Y. Kuwano, "Preparation and Properties of a-Si Films Deposited at a High Deposition Rate under a Magnetic Field," Jpn. J. Appl. Phys. Vol.27 (1988)40.

6. H. Chatham and P.K. Bhat, "High Deposition Rate P-I-N Solar Cells Prepared from Disilane Using VHF Discharges," Proc. Mater. Res. Soc. Vol.149(1989)447.

7. K. Kobayashi, M. Hayama, S. Kawamoto and H. Miki, "Characteristics of Hydrogenated Amorphous Silicon Films Prepared by Electron Cyclotron Resonance Microwave Plasma Chemical Vapor Deposition Method and their Application to PHotodiodes," Jpn. J. Appl. Phys. Vol.26(1987)202.

8. T. Kamimura, H. Nozaki, N. Sakuma, M. Nakajima and H. Ito, "Hydrogenated Amorphous Silicon Films Prepared by Mercury Sensitized Photochemical Vapor Deposition," Proc. Mater. Soc.Vol.149(1989) 51.

9. R.A. Street, D.K. Biegelsen, W.B. Jackson, N.M. Johnson and M. Stuzmann, "Dopant and Defect States in a-Si:H," Philosophical Magazine B, vol. 52 (1985)235.

10. Wei Guang-Pu, H. Okamoto and Y. Hamakawa, "Amorphous-Silicon Photovoltaic X-Ray Sensor," Jpn. J. Appl. Phys. Vol.24 (1985)1105.

11. K. Mochiki, K. Hasegawa and S. Namatame, "Amorphous Silicon PositionSensitive Detector," Nucl. Instr. and Meth. A273 (1988)640.

12. Y. Naruse and T. Hatayama, "Metal/Amorphous Silicon Multilayer Radiation Detectors," IEEE Trans. Nuc]. Sci. NS-36 (1989)1347.

13. H. Ito, S. Matsubara, T. Takahashi, T. Shimada and H. Takeuchi, "Integrated Radiation Detectors with a-Si Photodiodes on Ceramic Scintillators," Jpn. J. Appl. Phys. Vol.28 (1989)L1476. 
14. R.A. Street, S. Nelson, L.E. Antonuk and V. Perez-Mendez, "Amorphous Silicon Sensor Arrays for Radiation Imaging," Proc. Mater. Soc. Yol.192 (1990) 441.

15. S.N. Kaplan, J. Morel, V. Perez-Mendez and R.A. Street, "Detection of Charged Particles in Amorphous Silicon Layers," IEEE Trans. Nucl. Sci. NS-33 (1986) 351.

16. V. Perez-Mendez, S.N. Kaplan, G. Cho, I. Fujieda, S. Qureshi, W. Ward and R.A. Street, "Hydrogenated Amorphous Silicon Pixel Detectors for Minimum Ionizing Particles," Nucl. Instr. and Meth. A273 (1988) 127.

17. B. Equer and A. Karar, "Amorphous Semiconductors for Particle Detection: Physical and Technical Limits and Possibilities," Nucl. Instr. and Meth. A275 (1989) 558.

18. V. Perez-Mendez, G. Cho, J. Drewery, I. Fujieda, S.N. Kaplan, S. Qureshi, and R.A. Street, "Properties and Applications of Amorphous Silicon in Charged Particle, Gamma Ray and Light Detection," LBL-28339 (February 1990). To be published as a chapter in Physics and Applications of Amorphous and Microcrystalline Semiconductor Devices, Edited by J. Kanicki, Artech House, Boston, MA. Fall 1990.

19. C.A. Klein, "Bandgap Dependence and Related Features of Radiation Ionization Energies in Semiconductors," J. Appl. Phys. 39 (1968)2029. 


\section{Chapter 2. Signal generation in a-Si:H_radiation detectors}

\section{2-I. Introduction}

There was a concern about using a thick a-Si:H layer as a detector material because the low carrier mobilities and lifetimes might prevent collection of the radiation-induced electrons and holes in a limited measurement time. However, a higher bias can be applied on a-Si:H layers than on crystalline silicon since the material is highly resistive. To what extent this high bias can compensate the inferior transport properties of a- $\mathrm{Si}: \mathrm{H}$ is the problem addressed here. Problems associated with high bias opsration are discussed in Chapter 3. In this chapter, charge collection and signal generation in a-Si:H are analyzed to show that the material is capable of detecting radiation and to provide tools for detector design optimization.

In many device applications to reduce leakage current, the intrinsic a-Si:H layer is sandwitched between very thin $(30-50 \mathrm{~nm}) \mathrm{p}$ and $\mathrm{n}$ layers, making $\mathrm{p}-\mathrm{i}-\mathrm{n}$ diodes. (Other structures such as Shottky barrier and heterojunction diodes (using a-SiC:H, $\mathrm{Si}_{3} \mathrm{~N}_{4}$ as barriers) have been studied and a comparison of leakage currents for these structures can be found in ref.[1].) The p-i-n structure is schematically shown in Fig.2-1(a). Also shown in the figure are a charge-sensitive preamplifier and a shaping amplifier, standard electronics used with semiconductor radiation detectors. When such a p-i-n diode is reverse-biased, the movable carriers in the conduction and valence bands are swept away, leaving the positively charged dangling bond sites. Hence, the field profile is non-uniform i.e. linearly decreasing with distance in the first approximation for a uniform charge distribution [2]. Since the "intrinsic" a-Si:H is slightly n-type, depletion starts from the p-i interface. As the applied bias is increased, the depletion width $w_{d}$ increases. $w_{d}=\sqrt{ }\left(2 V_{d} \varepsilon_{s} / \rho\right)$, where $V_{d}, \varepsilon_{s}, \rho$ is the applied bias, dielectric constant and charge density, respectively. At some critical bias, the field extends to the n-i interface and full-depletion 
is achieved as shown schematically in Fig.2-1(b). This behavior of depletion can be observed by the following manner [3].

When a small amount of charge is injected at one end of the diode by shining a stronglyabsorbing light pulse on its face, the initial carrier distribution looks like that shown in Fig.2-1(c). One type of carrier is immediately collected by the nearby electrode and the other type of carrier traverses the depletion region. Hence, charge collection following a strongly-absorbing light pulse illumination is a single carrier process in a sense that only one type of carriers contributes to the output signal. Charges are induced on both electrodes by the drift motion of these carriers and a signal is generated in the attached charge-sensitive amplifier which integrates the induced current. A shaping amplifier is usually added to provide further amplification and also to improve count rate capability.

(a)

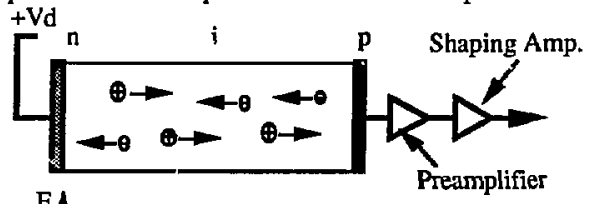

(b)

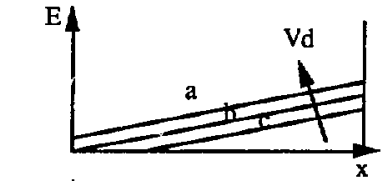

(c)

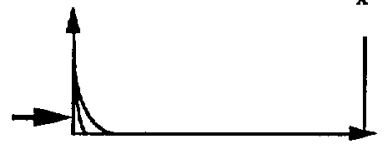

Fig.2-I (a) Schematic view of a reverse-biased diode and nuclear electronics. (b) Field profile inside the diode at some biases. (c) Initial distribution of photo-induced carriers by a strongly-absorbing light illumination on one end of the diode.

There is a distinct difference in signal development depending on which side of the diode is illuminated by the strongly-absorbing light pulse. When photo-carriers are injected at the $\mathrm{n}$-side, holes are the dominant coneributor to the signal. At low bias, the 
electric field does not extend to the n-side. Therefore, we would expect no signal until the full-depletion is achieved when the n-side is illuminated. As the bias is increased beyond the full-depletion bias, the signal should saturate since all the injected carriers complete transit through the diode. Thus the "hole signal" should show a threshold-type behavior as a function of bias. On the other hand, there should be a small signal at low bias when the p-side is illuminated because the field is non-zero at the p-i interface and is sufficient to drift electrons. At higher bias this "electron signal" would also saturate after the fulldepletion bias is reached.

\section{2-2. Theoretical analysis}

When a charge $q$ moves a small distance $d x$ in a diode of physical thickness $w$, it induces charges $d Q=q d x / w$ [4]. After a certain amount of charge $q\left(x_{0}, l_{0}\right)[C / c m ~ s e c]$ is introduced in the reverse-biased diode at a certain depth $x_{0}$ and at time $t_{0}$, the number of carriers decreases due to the deep trapping during the transit and its decrease is characterized by a lifetime $\tau$. The field displaces the remaining carriers, leading to the induced charge dQ on the parallel plate electrodes at time $t$, which is given by,

$$
\mathrm{dQ}=\frac{\mathrm{q}\left(\mathrm{x}_{0}, \mathrm{t}_{0}\right) \exp \left(-\frac{\mathrm{t}-\mathrm{t}_{0}}{\tau}\right)}{\mathrm{W}} \mathrm{dx}
$$

where $\mathrm{dx}$ is the displacement and $\mathrm{w}$ is the diode thickness. The current $\Delta \mathrm{i}$ due to this injected charge $q\left(x_{0}, t_{0}\right)$ generated in the external circuit at time $t$ is given by,

$$
\Delta i=\frac{d Q}{d t}=\frac{q\left(x_{0}, t_{0}\right) \exp \left(-\frac{t-t_{0}}{\tau}\right)}{w} \frac{d x}{d t}=\frac{q\left(x_{0}, t_{0}\right) \exp \left(-\frac{t-t_{0}}{\tau}\right)}{w} \mu E(x)
$$

So far, only the contribution from the charge $q\left(x_{0}, r_{0}\right)$ is considered. The total current $i(t)$ is obtained by adding all the contribution from the injected charges, namely,

$$
i(t)=\int_{0}^{w_{d}} d x_{0} \int_{0}^{t^{*}} d t_{0} \frac{q\left(x_{0}, t_{0}\right) \exp \left(-\frac{t-L_{0}}{\tau}\right)}{w} \mu E(x)
$$


where, the upper integral limit with respect to $x_{0}$ is the depletion width $w_{d}$. The upper limit with respect to $t_{0}$ denoted as $t^{*}$ here is either the transit time $t_{r}$ or the present time $t$, whichever is shorter. The transit time $t_{r}$ is given by integrating the following equation.

$$
\frac{\mathrm{dx}}{\mathrm{dt}}=\mu \mathrm{E}(\mathrm{x})
$$

Namely,

$$
\int_{x_{0}}^{x} \frac{d x}{\mu E(x)}=t-t_{0}
$$

Since $x=w_{d}$ (depletion width) at $t=t_{r}$,

$$
t_{r}=\int_{x_{D}}^{w_{d}} \frac{d x}{\mu E(x)}+t_{0}
$$

The total charge induced on the electrode during some measurement time $T_{m}$ after the charge injection is simply an integral of eq.(8) for electrons and holes, namely,

$$
\mathrm{Q}=\mathrm{Q}_{\text {electron }}+\mathrm{Q}_{\text {hole }}
$$

where

$$
Q=\int_{0}^{T^{*}} i(t) d t
$$

$T^{*}$ is either the measurement time $T_{m}$ or the time when all the carriers complete transit through the depletion layer whichever is shorter. Combining eq.(3)-(8), one can calculate the amount of charge collected during the measurement time $T_{m}$ for a known injected charge distribution and electric field profile $E(x)$.

Conversely, from the measured charge collection as a function of the bias, we can determine the field gradient, $i . e$. , the fixed space charge density, based on the known material parameters such as $\mu$ and $\tau$ and the injected charge distribution.

Although one can estimate charge collection in a diode with parallel plate electrodes for any given injected charge distribution by performing integrals shown above, it is instructive to derive an analytical expression for eq.(7) for a much simplified situation. 


\section{2-2.1. Instant charge creation at one end of a diode}

First, the case with the following assumptions is treated below: (a) the charge carriers are injected only at one end of a diode instantly at time 0 , (b) the field is linear through a diode, (c) it drops to 0 at the end of the depletion layer (abrupt junction approximation) and (d) carrier diffusion and dispersion are neglected. The current in eq.(3) becomes,

$$
\mathrm{i}=\frac{\mathrm{Q}_{\mathrm{0}}}{\mathrm{w}} \exp \left(-\frac{t}{\tau}\right) \mu \mathrm{E}(\mathrm{x})
$$

The collected charge $Q$ is obtained by integrating eq.(9) from time 0 to either the transit time of carriers or the measurement time. Notations used in this analysis are summarized in Table 2-1.

Table 2-1. Notation

\begin{tabular}{|l|l|l|l|}
\hline $\mathrm{Q}_{0}$ & total injected charge & $\mu$ & carrier mobility \\
\hline $\mathrm{w}$ & diode thickness & $\tau$ & carrier life time \\
\hline $\mathrm{w}_{\mathrm{d}}$ & depletion thickness & $\mathrm{N}_{\mathrm{d}}$ & ionizable defect density \\
\hline $\mathrm{V}_{\mathrm{d}}$ & applied bias & $\varepsilon_{\mathrm{s}}$ & dielectric constant \\
\hline $\mathrm{T}_{\mathrm{m}}$ & measurement time & \multicolumn{2}{|l}{} \\
\cline { 1 - 2 } & & &
\end{tabular}

When carriers are injected at the p-side of a diode, only electrons contribute to the signal. If a diode is only partially depleted, electrons cannot transit all through a diode, i.e., the measurement time is shorter than the transit time. Therefore, $T^{*}$ in eq.(8) is substituted by $\mathrm{T}_{\mathrm{m}}$ to obtain eq.(10). Just after the applied bias becomes high enough to fully-deplete a diode, the transit time is likely to be longer than the measurement time. So $T^{*}$ in eq.(8) is substitute by $T_{m}$ to obtain eq.(11). At higher bias, the transit time becomes shorter than $T_{m}$ and $T^{*}$ is substituted by the transit time to obtain eq.(12).

$$
\begin{aligned}
& Q=\frac{Q_{o} \mu}{w \beta} \sqrt{2 \alpha \nabla_{d}}\left[1-\exp \left(-\beta T_{m}\right)\right] \\
& Q=\frac{Q_{0} \mu}{\beta} E_{0}\left[1-\exp \left(-\beta T_{m}\right)\right] \\
& \text { for } V_{d} \leq V_{c} \\
& \text { for } \quad V_{c}<V_{d} \leq V_{c}
\end{aligned}
$$




$$
Q=\frac{Q_{0} \mu}{w \beta} E_{0}\left[1-\left[\frac{E_{0}-\alpha w}{E_{0}}\right]^{\beta / \alpha \mu}\right]
$$

where,

$$
\begin{aligned}
& \alpha=\frac{q N_{d}^{*}}{\varepsilon_{s}} \\
& V_{c}=\frac{\alpha}{2} w^{2} \\
& V_{c}=\frac{1+\exp \left(-\alpha \mu T_{m}\right)}{1-\exp \left(-\alpha \mu T_{m}\right)} V_{c} \\
& \beta=\alpha \mu+\frac{1}{\tau} \\
& E_{c}=\frac{V_{d}+V_{c}}{w}
\end{aligned}
$$

It is understood that the material parameters are those of electrons. From eq.(10) to eq.(12), the following behavior of the electron signal is evident: (a) the signal increases as a square-root of the bias until the diode is fully-depleted, (b) a shorter measurement time and a carrier lifetime both result in a smaller signal and (c) the signal saturates at $Q_{0}$ under the condition $\alpha \mu \gg>1 / \tau$ at high enough bias.

When carriers are injected at the $\mathrm{n}$-side of a diode, only holes can contribute to the signal as long as the field extends all through a diode. When a diode is partially depleted, holes can not drift at all, i.e., no signal. Just after the applied bias becomes high enough to fully-deplete a diode, the transit time is likely to be longer thar the measurement time. So $T^{*}$ in eq.(8) is substitute by $T_{m}$ to obtain eq.(18). At higher bias, the transit time becomes shorter than $T_{m}$ and $T^{*}$ is substituted by the transit time to obtain eq. (19).

$$
\begin{array}{lll}
\mathrm{Q}=0 & \text { for } & \mathrm{V}_{d} \leq V_{c} \\
\mathrm{Q}=\frac{Q_{0} \mu}{w \gamma} E_{00}\left[1-\exp \left(-\gamma T_{m}\right)\right] & \text { for } & V_{c}<V_{d} \leq V_{h} \\
Q=\frac{Q_{0} \mu}{w \gamma} E_{00}\left[1-\left[\frac{E_{00}+\alpha w}{E_{00}}\right]^{-\gamma / \alpha \mu}\right] & \text { for } & V_{h}<V_{d}
\end{array}
$$

where,

$$
V_{h}=\frac{\exp \left(\alpha \mu T_{m}\right)+1}{\exp \left(\alpha \mu T_{m}\right)-1} V_{c}
$$




$$
\begin{aligned}
& \gamma=-\alpha \mu+\frac{1}{\tau} \\
& E_{0 o}=\frac{V_{d}-V_{c}}{w}
\end{aligned}
$$

The material parameters $\mu$ and $\tau$ are those of holes. From eq.(17) to (19), the following behavior of the hole signal is evident: (a) the signal is zero until a diode is fully-depleted, (b) a shorter measurement time and a carrier lifetime both result in a smaller signal and (c) the signal saturates at $Q_{0}$ under the condition $\alpha \mu \gg 1 / \tau$ at high enough bias.

Charge collection efficiency $\left(Q / Q_{0}\right)$ can be calculated from eq.(10)-(12) for electrons and from eq.(17)-(19) for holes, respectively. An example plot as a function of bias is shown in Fig.2-2 for the fixed peaking time. Assumed parameters for this plot are as follows: $w=30 \mu \mathrm{m}, T_{\mathrm{m}}=9 \mu \mathrm{s}, \mu_{\mathrm{e}}=1 \mathrm{~cm}^{2} / \mathrm{Vs}, \mu_{\mathrm{h}}=0.005 \mathrm{~cm}^{2} / \mathrm{Vs}, \tau_{\mathrm{e}}=1 \mu \mathrm{s}, \tau_{\mathrm{h}}=2.5$ $\mu \mathrm{s}, \mathrm{N}_{\mathrm{d}}^{*}=7 \times 10^{+14} \mathrm{~cm}^{-3}$.

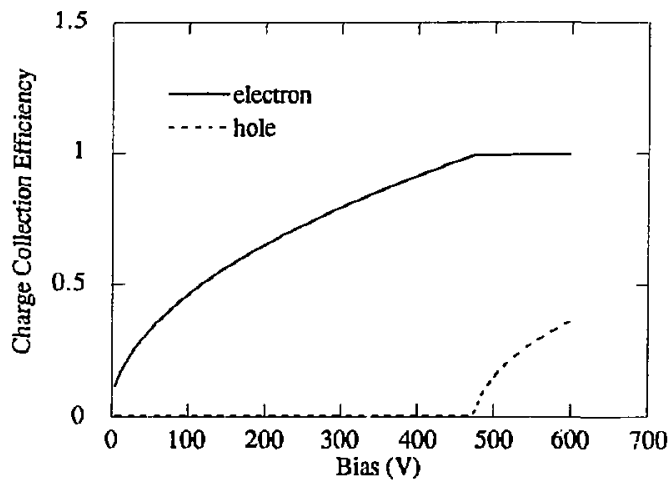

Fig.2-2 Calculated charge collection efficiencies for electrons and holes as a function of bias for a fixed peaking time of $9 \mu \mathrm{s}$. The assumes: diode parameters are given iit the text. 
Calculated efficiency as a function of the peaking time is shown in Fig.2-3 for a fixed bias. Assumed parameters for this plot are the same as Fig.2-2 except for the bias of 500 $V$ and the peaking time which is varied from 0 to $10 \mu s$.

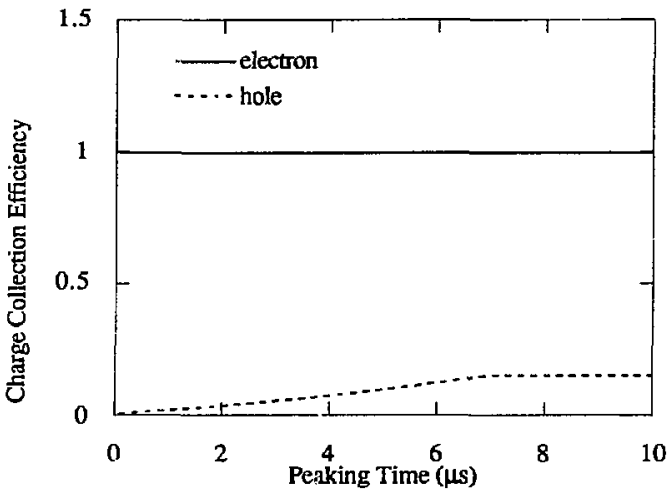

Fig.2-3 Calculated charge collection efficiencies for electrons and holes as a function of peaking time for a fixed bias of $500 \mathrm{~V}$. The assumed diode parameters are given in the text.

\section{2-2-2. Instant charge creation uniformly through a diode}

Both carriers centribute to the signal. For each carrier contribution, eq.(9) is integrated over the depletion width and over the transit time or the measurement time, whichever is shorter, namely,

$$
\mathrm{Q}=\frac{\mathrm{Q}_{\mathrm{o}}}{\mathbf{w}} \int_{0}^{\mathrm{w}_{\mathrm{d}}} \mathrm{dx_{0 }} \int_{0}^{\mathrm{T}^{*}\left(x_{0}\right)} \mathrm{dt} E \exp \left(-\frac{\mathfrak{l}}{\tau}\right)
$$

Now, the upper limit of the time integral depends on the location of the created charge. $Q_{0}$ is the injected charge per unit length of the diode. All the other notation is the same as before. With the same assumptions (b)-(d) in the previous case, one can perform 
integration analytically for each carrier type and for the cases of (i) partial depletion and (ii) deep depletion. In the partial depletion case, each carrier contribution is expressed as,

$$
\begin{aligned}
& Q_{c}=\frac{Q_{0} \mu_{e}}{c} V_{d}\left[1-\exp \left(-\beta_{e} T_{m}\right)\right] \\
& w \beta_{c} \quad \text { for } V_{d} \leq V_{c} \\
& \mathrm{Q}_{\mathrm{h}}=\frac{\mathrm{Q}_{\mathbf{o}} \mu_{\mathbf{h}}}{\mathbf{w} \gamma_{\mathbf{h}}} \mathrm{V}_{\mathbf{d}}\left[1-\exp \left(-\gamma_{\mathrm{i}} \mathrm{I}_{\mathrm{m}}^{\prime}\right)\right] \text { for } \mathrm{V}_{\mathbf{d}} \leq \mathrm{V}_{\mathbf{c}}
\end{aligned}
$$

It should be noted that the signail increases linearly with bias. To perform integration for the deep depletion case, the integral range of position $x_{0}$ is sub-divided into two ranges, the region where the transit time is shorter than the measurement time and the region where collection is limited by the measurement time. The boundary of these two regions depends on the bias. The resultant analytical expressions for the deep depletion case are complicared ard don $t$ give much additional insight on the signal behaviors other than that it saturates at high enough bias.

\section{2-3 Measurement}

Three light emitting diodes (LED) were chosen to give light pulses of three different wave'ingths. Selection of LFDs was based on their wavelength and speed. Absorption coefficient of a-Si:H at various wavelength can be found in Chapter $\mathrm{l}$ of ref.[1]. The mean free path for absorption is calculated from this and shown in Fig.2-4. This will help us to choose wavelength of the light source for the experiments described in this chapter. 


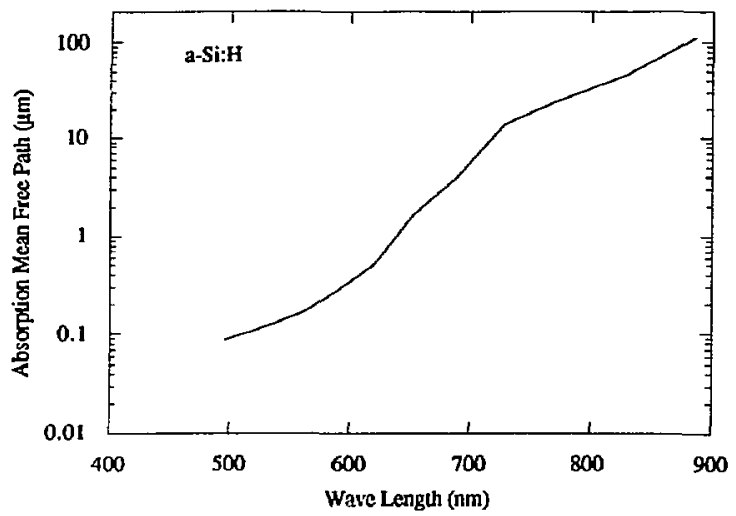

Fig.2-4 Absorption mean free path in a-Si:H at various wavelength.

The Hewlett-Packard LED (type HFBR-1510) was relatively fast ( $50 \mathrm{nsec}$ rise time) and strongly absorbed at the surface $(665 \mathrm{~nm})$. The General Electric LED (type F5E1, $880 \mathrm{~nm}$ peak) was uniformly absorbed through a-Si:H but slow (1.5 $\mu \mathrm{sec}$ rise time). The Hitachi LED (type HLP40RA) was fast ( 20 nsec rise time) and gave intermediate wavelength (760 $\mathrm{nm}$ ). These were easily available LEDs and gave light pulse intense enough for easy measurement, but were not necessarily the best choice. For example, shorter wavelength and faster light pulse would give better results which are easier to interpret as discussed later. The mean-free-path of these light sources in a-Si:H ranges from about $3 \mu \mathrm{m}$ (665 $\mathrm{nm}$ light) to about $100 \mu \mathrm{m}$ (880 $\mathrm{nm}$ light). The a-Si:H sample was a $27 \mu \mathrm{m}$ thick p-i-n diode made by GSI. Its structure was Corning 7059 glass substrate/Tin oxide (transparent electrode) $/ \mathrm{p}^{+}$-type a-SiC:H/i-type a-Si:H/n+-type a-Si:H/semi-transparent Cr electrode. This is a typical a-Si:H solar-cell structure except for the extremely thick $\mathrm{i}$ layer. A charge sensitive amplifier and a quasi-Gaussian shaping amplifier (variable peaking time 0.1-9 
$\mu$ sec) were instalied to detect the photo-induced charges which were kept small enough not to distort the field established by the external bias.

Two parameters of the experiment were the reverse-bias and the peaking time. First, the bias was varied for a fixed peaking time to measure charge collection efficiency as a function of bias. Second, the peaking time was varied for a fixed bias to measure how fast the carriers are collected. In all cases shown below, the collected charges were kept small enough not to distort the existing field by adjusting the intensities of LED outputs.

\section{2-3-1. Signal size dependence on a bias}

The results of the first measurement are shown in Fig.2-5. The light pulsing conditions were $100 \mathrm{nsec}$ pulse width for the $665 \mathrm{~nm}$ and $760 \mathrm{~nm}$ LEDs and about $3 \mu \mathrm{sec}$ width for the $880 \mathrm{~nm}$ LED. Either the $\mathrm{n}$ or $\mathrm{p}$ side of the diode was illuminated by these light pulses with repetition rates ranging from $60 \mathrm{~Hz}$ to $600 \mathrm{~Hz}$. Charge collection corresponding to a single light pulse was recorded as a function of the diode bias with the fixed peaking time of $9 \mu \mathrm{sec}$. It was found that the signal size depends also on the time after the bias is applied. The signal decreased first rapidly after the bias application, but it slowly reached an asymptotic value. The highest bias was applied to the diode first and the signal was recorded after a certain time when this equilibrium was reached. Subsequent data were taken by reducing the bias, thus avoiding this transient. This behavior of time dependence will be discussed further in section 2-3-3. The recorded signal size was normalized in the following manner: First, the $880 \mathrm{~nm}$ signals at the highest bias for both $\mathrm{p}$ and $\mathrm{n}$-side illumination was set to 1.0 . Because the $880 \mathrm{~nm}$ light is assumed to produce uniform injection through the diode independent of the direction of incidence, this normalization process corrects the difference in the amount of injected charges for $\mathrm{p}$ and $\mathrm{n}$-side illumination, assuming that the transparency of the contact electrode and heavily-doped layer for the light is the same. The normalization constant for this process was used to scale the $665 \mathrm{~nm}$ and $760 \mathrm{~nm}$ signal for each case of $\mathrm{p}$ and $\mathrm{n}$-side 
illumination. Finally, for each of $665 \mathrm{~nm}$ and $760 \mathrm{~nm}$ signal, signal size was scaled with respect to the signal size of $\mathrm{p}$-side illumination at the highest bias

The signals produced by the detection of the $880 \mathrm{~nm}$ and the $760 \mathrm{~nm}$ light pulses are shown in Fig.2-6 as a function of the applied bias for a fixed peaking time of $9 \mu \mathrm{s}$.

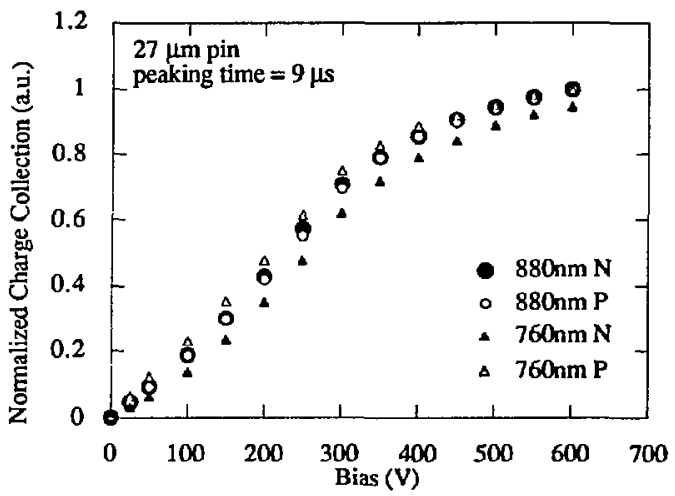

Fig.2-5 Charge collection as a function of the applied bias for detecting uniformlyabsorbing light pulses.

It can be seen that charge collection for the p-side illumination is almost identical to that of the $\mathrm{n}$-side illumination for the $880 \mathrm{~nm}$ light, confirming the uniform charge creation throughout the diode. The initial rise of these signals is linear with bias as predicted by eq.(24)-(25). As for the $760 \mathrm{~nm}$ light, the agreement between the p-side and the $\mathrm{n}$-side illumination is less complete as expected from its shorter mean free path for absorption (about $30 \mu \mathrm{m}$ ).

The signals produced by the detection of the $665 \mathrm{~nm}$ light pulses are plotted in Fig.2-6. 


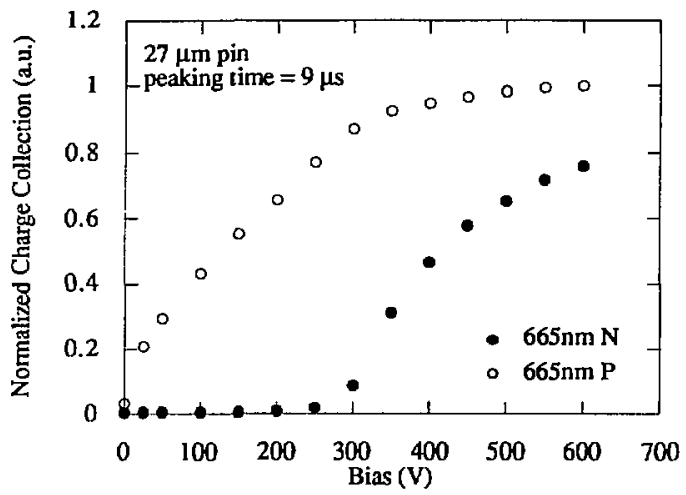

Fig.2-6 Charge collection as a function of the applied bias for detecting strongly-absorbing light pulses.

Now there is a distinct difference bettween the two cases. The "threshold behavior" was clearly seen in Fig.2-6 for the n-side illumination by the $665 \mathrm{~nm}$ light pulse (hole signal). There was not such a behavior for the p-side illumination (electron signal) and it started to saturate at the "threshold bias" of the hole signal. The increase of the electron signal at low bias is proportional to the square-root of bias as predicted approximately by eq. (10).

\section{2-3-2. Signal size dependence on peaking time}

Next, the bias was fixed at $500 \mathrm{~V}$ and the peaking time was varied. This bias was chosen to measure the peaking time dependency at the operational bias. The same pulsing conditions of LEDs to obtain Fig.2-5 and Fig.2-6 were used except the pulse width was reduced to $50 \mathrm{nsec}$. The $880 \mathrm{~nm}$ LED was not used due to its limited speed. Signal size was normalized according to the corresponding data in Fig.2-6 at $500 \mathrm{~V}$ of bias and $9 \mu \mathrm{sec}$ of peaking time. 


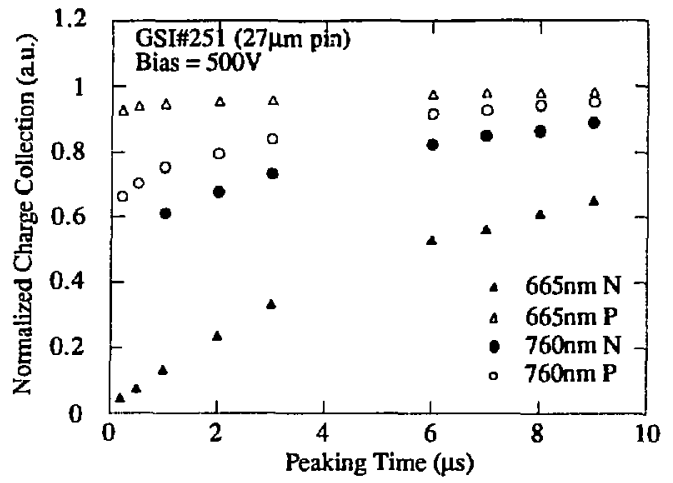

Fig.2-7 Charge collection as a function of the peaking time.

Figure 2-7 shows that more than $90 \%$ of electrons $(665 \mathrm{~nm} \mathrm{P})$ were collected within less than $100 \mathrm{nsec}$ at this bias although the hole collection $(665 \mathrm{~nm} \mathrm{~N})$ was much slower. This can be attributed to the small hole mobility as well as the dispersive nature of the hole transport. The $760 \mathrm{~nm}$ signal is due to the mixture of both carriers and it shows an intermediate behaviour between the electron and hole signal.

\section{2-3-3. $\mathrm{Nd}^{*}$ dependence on time after bias application}

The signal size dependence on time after bias application was investigated further. It is related to the field profile dependence on the time, which is determined by the ionized defect density $\mathrm{N}_{d}{ }^{*}$. It is known in a-Si:H that defect (dangling bonds) states are located in the middle of the band gap and that they release electrons under DC bias to become fixed positive space charges [2]. The thermal excitation rate of electrons from the dangling bond sites to the conduction band is given by $\omega_{0} \exp (-\Delta E / \mathrm{kT})$ where $\omega_{0} \approx 10^{+12}-10^{+13} \mathrm{sec}^{-1}$ and $\Delta \mathrm{E} \approx 0.8 \mathrm{eV}[2] . \quad N_{\mathrm{d}}^{*}$ increases with time after the bias application and reaches an 
equilibrium value when the emission rate of electrons becomes equal to the emission rate of holes. This transient regime of $\mathbf{N}_{d}$ " provides information about the gap state distribution in the energy band as shown by Matsuura [5]. The Time of Flight (TOF) measurements [2] were repeated at various times after bias application to measure the time dependency of $\mathrm{N}_{\mathrm{d}}{ }^{*}$. A detailed description of the TOF measurement and analysis is given in the Appendix at the end of this chapter.

The TOF signals following a 3 ns wide $510 \mathrm{~nm}$ Laser light pulse illumination on a DC biased a-Si:H p-i-n diode are shown in Fig.2-8 for each time after bias application. There is a change in the slope of the transient photocurrents in Fig.2-8 (a) and (b). This exponential decay of the transient photocument gives the ionized defect density as shown in the Appendix. This was calculated for each time after bias application and the result is shown in Fig.2-9. The equilibrium is reached about 6-7 min after the bias is applied.

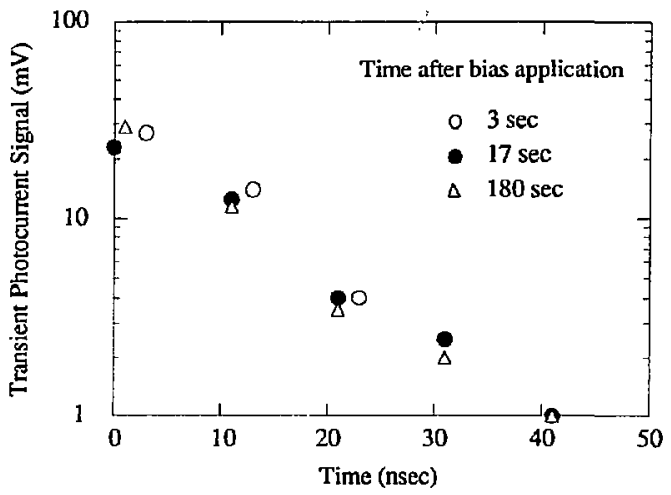

(a) 


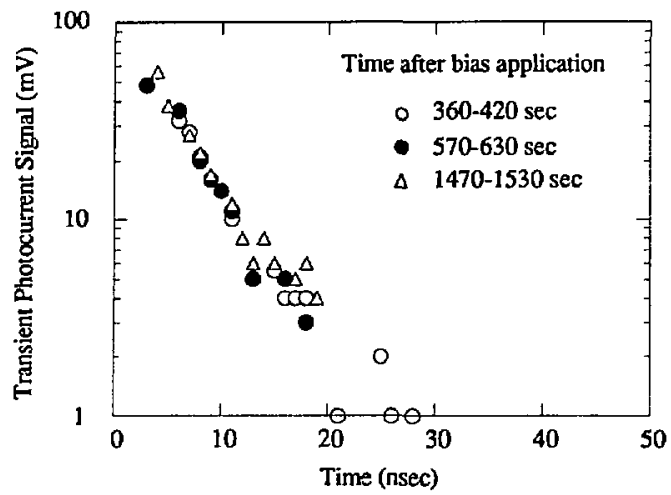

(b)

Fig.2-8 Successive TOF measurements for various times after bias application. Photocurrents following a 3 ns wide $510 \mathrm{~nm}$ Laser light pulse illumination on a DC biased a-Si:H p-i-n diode are shown for different times after bias application.

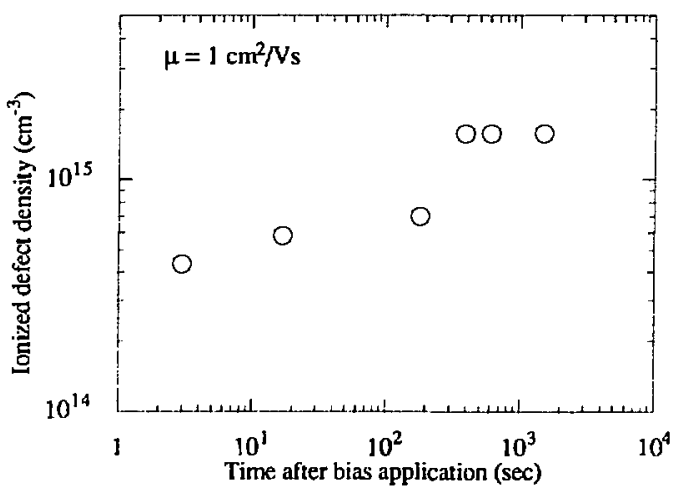

Fig.2-9 $\mathrm{N}_{\mathrm{d}}$ * calculated from the successive TOF measurements shown in Fig.2-8. Electron mobility of $1 \mathrm{~cm}^{2} / \mathrm{Vs}$ was assumed. 


\section{2-4. Discussion}

In Fig.2-5 and Fig.2-6, the expected behaviors of signals induced by these LED light pulses are clearly observed as a function of the bias. Namely, the electron signal shows square-rcot dependency on the bias at low bias and tends to saturate at higher bias, whereas the hole signal shows the "threshold" behavior. The signals from the uniformly-absorbing light pulses show linear dependency on the bias. The expected peaking time dependency of the collection efficiency is observed as well in Fig.2-7. The electron signal is already saturated at the bias and peaking time range used whereas the hole signal slowly increases with the peaking time. The discrepancy between the measurements and the calculations may be jue to the fact that the dispersive nature is not taken into account for the hole signal analysis.

Since the onset of the hole signal gives valuable information about the ionized defect density $\mathrm{N}_{\mathrm{d}}{ }^{*}$, this signal was analyzed further by the theoretical expression given by eq.(17)-(19). The result is shown in Fig.2-10 together with the hole mobility and liferime measured separately by the TOF analysis [6] with a much shorer pulse of $510 \mathrm{~nm}$ Laser light. The theoretical expression can fit the shape of the measured data fairly well. However, the more precise TOF analysis gave $\mathrm{N}_{d}^{*}$ of $7 \times 10^{+14} \mathrm{~cm}^{-3}$ for this diode [6]. The discrepancy between the TOF and this analysis presumably comes from the wavelength of "strongly-absorbing" light. The $665 \mathrm{~nm}$ light penetrates the a-Si:H layer further than the $510 \mathrm{~nm}$ light and the assumption of surface absorption in deriving eq.(17)(19) is less valid for the $665 \mathrm{~nm}$ light. 


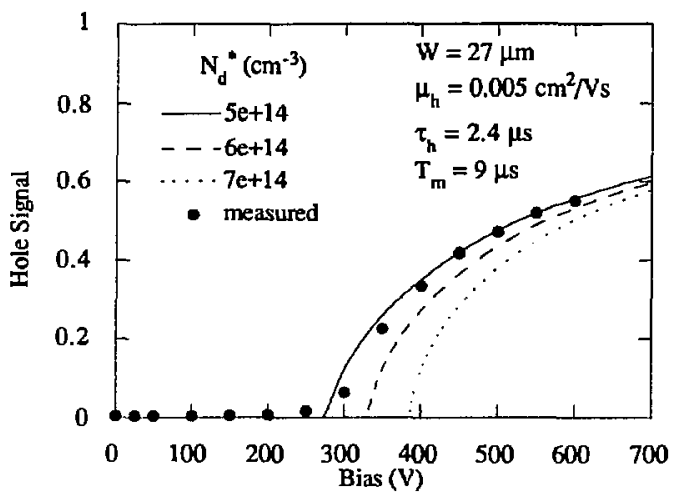

Fig.2-10 Hole signal fit by the theoretical expression (eq.(17),(18),(19)). A value of $7 x$ $10^{+14} \mathrm{~cm}^{-3}$ for $\mathrm{N}_{\mathrm{d}}$ was obtained by the TOF measurement. See text.

\section{2-5. Conclusion}

The charge collection and signal generation process in a-Si:H was investigated using a short light pulse from LEDs. It shown that a thick a-Si:H layer can be biased high enough to ensure good charge collection efficiency. Electron collection takes place in a short time but hole collection takes longer and this results in signal loss when a peaking time less than a few $\mu$ s is used. The theoretical expressions for the signal provide information about material parameters as well as tools for detector design optimization.

Two possible improvements to these experiments are both related to the light source. First, a shorter wavelength light is more strongly absorbed on the surface and the photocarriers represent a better sheet-like spatial distribution. This results in a better "threshold behavior," i.e., the onset of the hole signal in Fig.2-6 becomes sharper. Second, using a faster LED or Laser can decrease the lower limit of peaking time in Fig.2-7. 


\section{Appendix Time of Flight analysis}

Charge collection analysis described in this chapter is a quick test of depletion in a-Si:H diodes and it assumes that the basic material parameters such as mobility and lifetime are already known. These parameters are often deposition-dependent and are obtained by some characterization techniques, most notably by the Time of Flight (TOF) method. In this appendix, how one can calculate mobility, lifetime and field profile is described.

\section{A1. Time of Flight Basics}

Figure A1 shows the basic setup of the TOF method for the carrier mobility measurements. It consists of a pulsed Laser as a light source and transient current recording system. A wide-band amplifier is used to observe the voltage drop across the 50 $\boldsymbol{\Omega}$ resistor and the clipping diodes are provided for amplifier protection. Photocarriers are injected by a strongly absorbing pulsed laser light through a semi-transparent electrode into an a-Si:H diode. One sign of carriers is drifted througin the diode by the electric field created by the external bias. The other sign of carriers is immediately collected at the electrode and do not contribute to the photocurrent. The transient photocurrent due to the one type of carrier is recorded by the digitizing oscilloscope. From the measured transit time $t_{r}$ and the known diode thickness $w$ and applied bias $V$, the carrier mobility $\mu$ is calculated by the simple relation,

$$
\mu=\frac{w^{2}}{V_{t_{r}}}
$$

The following precautions about the experiment conditions must be made for meaningfull and clean results.

(i) The laser light must be absorbed strongly near the surface to make the initial carrier distribution close to a sheet. A wavelength of $510 \mathrm{~nm}$ is commonly used. The absorption depth of a-Si:H at this wavelength is less than $1 \mu \mathrm{m}$. 
(ii) The amount of the injected carriers $Q$ must be much smaller than the "CV limit", where $\mathrm{C}$ is the capacitance of the diode and $\mathrm{V}$ is the applied bias, in order to ensure that the existing field is not disturbed by the drifting space charge. The often used criterion is, $\mathrm{Q}<\frac{\mathrm{CV}}{10}$

(iii) The external bias must be applied shortly before the photoinjection to ensure that the field is uniform before and during the carrier transit. This delay time used in the experiment described in section 2-3-3 was $50 \mu \mathrm{s}$.

(iv) For repeated measurements by pulsing the bias and the laser, the frequency must be low to eliminate possible contribution from the trapped carriers which are released during the subsequent photoinjection. Two successive laser pulses were separated by at least $10 \mathrm{sec}$ in the experiments described in section 2-3-3.

Some practical precautions about the setup are as follows:

(i) The system must have enough time resolution. The main components of our ordinary TOF setup consist of a $150 \mathrm{MHz}$ scope (Tektrinix 2430) and a laser with $3 \mathrm{nsec}$ pulse duration. This is good enough if we sample a number of pulses because the scope can fill the digitization gaps by multiple samplings, essentially increasing the sampling density. For a single pulse measurement as in the case of section 2-3-3, only one sampling is done every $10 \mathrm{~ns}$. A higher sampling is desirable for more accurate measurements.

(ii) The scope is trigered by the fast signal from the crystalline photediode. The driving pulse of the $\mathrm{N}_{2}$ laser should not be used for this purpose because laser pulsing has time fluctuation.

(iii) The diode and the signal pick-up line must be well shielded from the outer world noise especially from the pulsing laser. A separate shielding box with a metal mesh on the laser input window is helpful. The filter for the bias should be kept close to the diode.

(iv)The product of the contact resistance $\mathrm{R}$ and the capacitance $\mathrm{C}$ of a diode must be small compared with the transit time because it slows down the transient signal. Typically, 
rise time less than $10 \mathrm{~ns}$ was obtained with the $3 \mathrm{~ns}$ laser pulse and $10-50 \mu \mathrm{m}$-thick diodes with $3 \mathrm{~mm}$-diameter $50 \mathrm{~nm}$-thick $\mathrm{Cr}$ contacts.

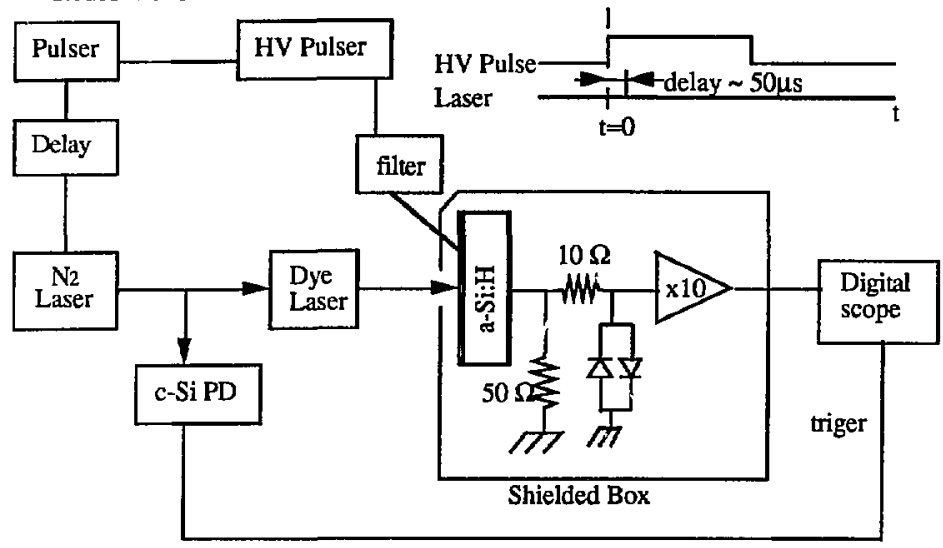

Fig.A1 Time of Flight setup

A carrier lifetime $\tau$ is another important parameter of the material and the product $\mu \tau$ is estimated by the so-called Hecht analysis. Here, the integrated photocurrent is recorded as a function of the bias. At the low bias, some carriers are lost due to trapping during the transit and cannot contribute to the charge collected. The carrier density $n$ decreases from the initial value no according to the relation,

$$
\mathrm{n}=\mathrm{n}_{\mathrm{o}} \mathrm{e}^{-\mathrm{v} \tau}
$$

The induced charge on the electrode due to the drifing charges $n$ is given by

$$
\mathrm{dQ}=\mathrm{n} \frac{\mathrm{qdx}}{\mathrm{w}}=\frac{\mathrm{n}_{\mathrm{o}} \mathrm{q}}{\mathrm{w}} \mathrm{e}^{-\psi / \tau} \mathrm{dx}
$$

The total charge induced $Q$ during the transit is,

$$
Q=\frac{n_{0} q}{w} \int e^{-t / \tau} d x
$$

This integral is converted to the time integral from the relation $\mathrm{dx} / \mathrm{dt}=\mu \mathrm{V} / \mathrm{w}$ and is performed from time 0 to the transit time $t_{r}$. The result is, 


$$
Q=\frac{n_{0} q V}{w^{2}} \mu \tau\left(1-e^{-4 / \tau}\right)
$$

Hence, the $\mu \tau$ product is given by fitting the $Q V$ curve for $t_{q}>\tau$.

\section{A2. DC bias TOF}

The fixed space charge density $\mathrm{N}_{\mathrm{d}}{ }^{*}$ in an ạ-Si:H diode under $\mathrm{DC}$ bias is estimated from the electron transient photocurrent. Electron transient is prefered to hole transient which often shows dispersive nature.

\section{Constant $\mathrm{N}_{\mathbf{d}}$ ( case (simplified picture)}

To show the functional dependence of electron transient photocurrent on time, the following simplified analysis may be helpful. First, the electric field can be assumed linear under the DC bias condition so that we have the following expression for the field profile.

$$
E(x)=\frac{\mathrm{qN}_{\mathrm{d}}^{*}}{\varepsilon_{\mathrm{S}}} \mathrm{x}_{-} \mathrm{E}_{\mathrm{O}}
$$

Here, $\mathrm{E}_{\mathrm{O}}$ is the field strength at the surface where the photoinjected charges are located $(x=0)$ initially $(t=0)$. Noting that electrons are negatively charged, the electron drift velocity is then given by,

$$
\frac{d x}{d t}=-\mu E(x)=-\frac{q \mu N_{d}^{*}}{\varepsilon_{S}} x+\mu E_{o}
$$

This 1 st order ordinary differential equation can be solved with the initial condition $x=0$ at $t=0$ to give the following solution.

$$
x=\frac{\varepsilon_{s}}{q N_{d}^{*}} E_{o}\left(1-e^{-\frac{q \mu N_{d}}{\varepsilon_{d}}} \mathrm{t}\right)
$$

The field profile is rewritten as,

$$
E(x)=\frac{\mathrm{qN}_{\mathrm{d}}^{*}}{\varepsilon_{\mathrm{S}}} \frac{\varepsilon_{\mathrm{s}}}{\mathrm{qN}_{\mathrm{d}}^{*}} E_{\mathrm{o}}\left(1-\mathrm{e}^{-\frac{\mathrm{q} \mathrm{w} N_{\mathrm{d}}}{\varepsilon_{0}}} \mathrm{l}\right) \cdot \mathrm{E}_{\mathrm{o}}
$$

The induced charge on the electrode by $n$ electrons drifting through the parallel-plate electrodes of separation $w$ is given by,

$$
\mathrm{dQ}=\frac{\mathrm{nqdx}}{\mathrm{w}}
$$


The current is given by,

$$
\mathrm{i}(\mathrm{t})=\frac{\mathrm{dQ}}{\mathrm{dt}}=\frac{\mathrm{nq} \frac{\mathrm{dx}}{\mathrm{dt}}}{\mathrm{w}}=-\frac{\mathrm{nq}}{\mathrm{w}} \mu \mathrm{E}(\mathrm{x})
$$

Substituting the expression of $\mathrm{E}(\mathrm{x})$ in the above equation, we get,

$$
\mathrm{i}(\mathrm{t})=\frac{\mathrm{nq}}{\mathrm{w}} \mu \mathrm{E}_{\mathrm{o}} \mathrm{e}^{-\frac{q \mu N_{d}}{\mathrm{E}_{\mathrm{d}}}}
$$

This simple analysis shows that the transient photocurrent diminishes exponentially with time under the DC bias condition. The slope of the $\ln \mathrm{i}(\mathrm{t})$-t plot is proprotional to $\mathrm{N}_{\mathrm{d}}{ }^{*}$.

The measured transient current has a somewhat modified shape at the beginning of the curve due to the built-in potential close to the electrode and/or the finite response time of the measuring system. However, we can identify the time range during which the transient current decays exponentially when the transient photocurrent is plotted in the semi-log scale. This range corresponds to the mage of the linearly changing field. We can estimate $\mathrm{N}_{\mathbf{d}}{ }^{*}$ by the following relation.

$$
N_{d}^{*}=\frac{\varepsilon_{s}}{q \mu} \frac{\ln \left(i\left(t_{1}\right) / i\left(t_{2}\right)\right)}{t^{-t_{1}}}
$$

\section{Non-constant $\mathbf{N}_{\mathrm{d}}^{*}$ case (Field profiling)}

The method described above for the constant $N_{d}{ }^{*}$ case can be extended to the nonconstant $\mathrm{N}_{\mathrm{d}}{ }^{*}$ case, namely, the electric field profile of a reverse-biased diode can be obtained from the analysis of the transient photocurrent under the DC bias condition.

The transient photocurrent under the DC bias condition is related to $\mathrm{E}(\mathrm{x})$ by eq.(A12).

$$
i(t)=\frac{d Q}{d t}=\frac{n q \frac{d x}{d t}}{w}=-\frac{n q}{w} \mu E(x)
$$

The measured quantity is $i(t)$. So the field profile $E(x)$ is given by a function of time. Converting the variable $t$ to $x$ is done by the relation,

$$
x=\int_{0}^{t}-\mu E(x) d t=\frac{w}{n q} \int_{0}^{t} i\left(t^{\prime}\right) d t^{\prime}
$$

Each value of $t$ corresponds to 'sach value of $x$ by the above equation. Rescaling eq.(A12) by eq.(A15) gives the field profile $\mathrm{E}(\mathrm{x})$. 
Once the field profile $\mathrm{E}(\mathrm{x})$ is estimated by above equations, the spatial distribution of the space charge density $N_{d}^{*}(x)$ is obtained by $N_{d}^{*}(x)=\left(\varepsilon_{j} / q\right) d E(x) / d x$.

The anomaly of $N_{d}{ }^{*}(x)$ within a few $\mu \mathrm{m}$ of the diode ends obtained by this method is likely due to the built-in potential as well as due to the real fluctuation in $\mathrm{N}_{\mathrm{d}}{ }^{*}$. 


\section{References}

1. K. Kempter, "a-Si:H Image Sensor: Some Aspects of Physics and Performance," SPIE Vol.617 (1986)120.

2. R.A. Street, "Measurements of Depletion Layers in Hydrogenated Amorphous Silicon," Physical Review B 27 (1983)4924.

3. I. Fujieda, G. Cho, S.N. Kaplan, V. Perez-Mendez, S. Qureshi, W. Ward and R.A. Street, "Detection of Charged Particles in Thick Hydrogenated Amorpinous Silicon Layers," Proc. Mater. Res. Soc. Vol.118 (1988)469.

4. P.A. Tove and K. Falk, "Pulse Formation and Transit Time of Charge Carriers in Semiconductor Junction Detertors," Nucl. Instr. and Meth. 29 (1964)66.

5. H. Matsuura, "A Novel Method for Determining the Gap-State Profile and its Application to Amorphous $\mathrm{Si}_{1-x} \mathrm{Ge}_{x}: H$ Films," J. Appl. Phys. 64 (1988)1964.

6. S. Qureshi, V. Perez-Mendez, S.N. Kaplan, I. Fujieda, G. Cho and R.A. Street, "Signal Generation in a Hydrogenated Amorphous Silicon Detector," IEEE Trans. Nucl. Sci. NS-36 (1989)194. 


\section{Chapter 3. Field profile tailoring}

\section{3-1. Introduction}

To detect radiation directly, intrinsic a-Si:H layers considerably thicker than in most of other applications of a-Si:H are desirable. Diodes of simple structures such as Schottky barrier or $\mathrm{p}-\mathrm{i}-\mathrm{n}$ with $5-50 \mu \mathrm{m}$ thick inirinsic layers have been fabricated and tested successfully with charged particies, $X$-rays and $\gamma$ rays. Due to its low carrier mobility and lifetime, a high bias must be applied to ensure good charge collection efficiency as discussed in Chapter 2.

However, the highest reverse-bias applicable to these diodes is limited to about $10-20 \mathrm{~V}$ times the thicknesses of diodes in $\mu \mathrm{m}$ (or an average field of $10-20 \mathrm{~V} / \mu \mathrm{m}$ ) by the onset of a "pop-corn" type noise [1,2]. On oscilloscope observation of the shaping amplifier output, this noise has a burst-like, intermittent behavior. Typical noise and current-bias (I-V) characteristics for some p-i-n a-Si:H diodes as measured with standard nuclear electronics with peaking time $6.4 \mu \mathrm{s}$ is shown in Fig.3-1. These p-i-n diodes had 16-17 $\mu \mathrm{m}$ thick intrinsic layer with thin $(30-50 \mathrm{~nm})$ doped layers. The top contact was a $3 \mathrm{~mm}$ diameter disk-shaped evaporated $\mathrm{Cr}$. The boltom contact was about $1 \times 1 \mathrm{~cm}^{2}$ evaporated $\mathrm{Cr}$. Both electrodes were about $500 \AA$ thick. These samples were cut from a larger piece of aSi:H layer deposited on a $0.8 \mathrm{~mm}$ thick 4' $\times 4^{\prime}$ Corning 7059 glass coated with $\mathrm{Cr}$. The noise onset at high bias is not associated with current increase and therefore neither the shot noise [3] nor the $1 / \mathrm{f}$ noise [4] is responsible for this sudden noise increase. Moreover, there is a wide variation in this highest bias even among the samples made in a single deposition run as shown in this example shown in Fig.3-1. This proposes a serious problem of nonuniformity for large area applications.

A possible explanation to this phenomenon is the microplasma breakdown $[5,6]$ in the vicinity of the diode surface or the $\mathrm{p} / \mathrm{h}$ interface where the field strength becomes maximum. Since the $p$ layer is very thin, any material inhomogeneity close to the surface increases the 
chance of local breakdown. The local field created by the material inhomogeneity can reach the critical field for avalanche multiplication temporarily and then the space charges created by the avalanche shield the external field, suppressing the avalanche. As the space charges are swept away, the high local field is established again by the material inhomogeneity and this avalanche-field collapsing cycle continues. Although this hypothetical mechanism is an interesting topic to investigate, a more practical requirement is to find how to suppress this noise onset at high bias or perhaps to harness it for avalanche multiplication. If we can control the field profile in a diode, the local field in the vicinity of surfaces can be suppressed while keeping high field in the majority of the diode.

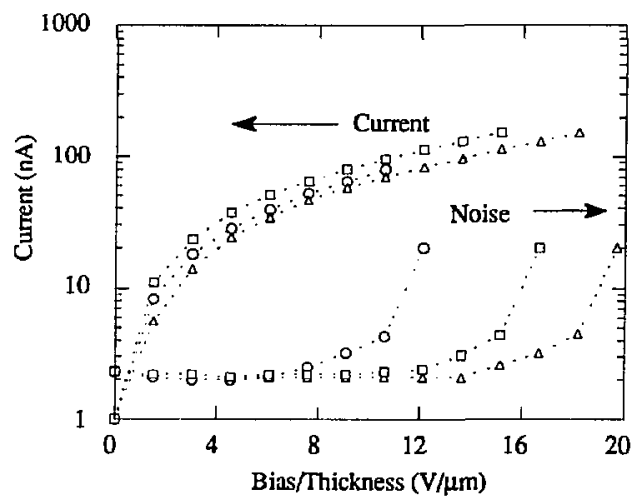

$10^{6}$

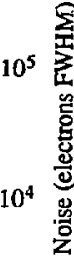

$10^{3}$

\section{Bins/Thickness (V/um)}

Fig.3-1 I-V and noise characteristics of three representative p-i-n diodes. These p-i-n diodes had 16-17 $\mu \mathrm{m}$ thick intrinsic layers sandwiched by thin (30-50 $\mathrm{nm}$ ) doped layers and they were made from a single deposition run. The peaking tome of the shaping amplifier was $6.4 \mu \mathrm{s}$.

Another problem associated with a thick a-Si:H layer with parallel plate electrodes is the field gradient determined by the ionized defect density $N_{d}^{*}$ as discussed in Chapter 2 . Unless the material quality is improved dramatically, the field gradient in a-Si:H layers may prevent full depletion of a diode under the highest reverse bias possible before the noise 
onset. It would be advantageous to control the field profile which determines carrier transport.

In this chapter, the idea of tailoring the field profile is introduced to suppress the noise onset as well as to compensate for the effect of the field gradient. Two methods of manipulating the field profile to achieve these objectives, via doping and electrode geometry, are discussed. Field manipulation by doping utilizes the fixed space charges of the ionized donor and acceptor atoms in the doped layers. The characteristics of a "buried $p-i-n "$ structure will be described in detail. Charge collection for $\alpha$ particle detection is shown to improve by the higher field achieved in this structure. A simple charge collection model for detecting a high LET particle with a low-mobility semiconductor material is developed to account for the charge collection process observed in $\alpha$ particle detection. Other structures such as making thicker doped layers in the $p-i-n$ structure are also described.

\section{3-2. Tailoring by doping}

Doping a-Si:H is most commonly achieved by mixing gases during deposition. Abrupt compositional change at interfaces between doped layers and intrinsic layer is possible under this condition. Superlattice structure by doping modulation has been extensively investigated. Based on the same production method, field profile tailoring in a thick aSi:H layer becomes possible.

\section{3-2-1. Field profile calculation for multilayer structures}

Field profiles of some doping-modulated multilayer structures are calculated by solving the Poisson equation in each layer and connecting each solution at each boundary. The assumptions for simplification of this mathematical problem are as follows. A parallel plate electrode configuration is assumed so that the Poisson equation reduces to an one 
dimensional equation. Only the full-depletion case is treated here and charge density in each layer is assumed to be constant. The field in each layer is therefore linear and its gradient is proportional to the fixed space charge density arising from the doping atoms and the ionized dangling bonds.

Figure 3-2 shows a schematic multilayer structure. The space-charge density and thickness of each layer is denoted as $N_{i}$ and $x_{i}$, respectively. Under the depletion approximation, the space-charge density $\mathrm{N}_{\mathrm{i}}$ is assumed to be equal to the donor or acceptor density in the case of doped layers and to the ionizable defect density in the case of intrinsic layers under bias. This assumption is only valid where the bias is high enough to fullydeplete the whole layer and injected carrier density is negligible compared to $\mathrm{N}_{\mathrm{i}}$.

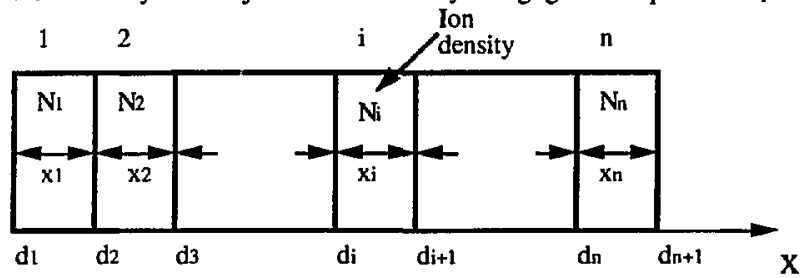

Fig.3-2 Multilayer structure

The Poisson eq.(1) is solved in each layer and each solution is connected at boundaries using the continuity conditions eq.(2).

$$
\begin{array}{ll}
\frac{d^{2} V_{i}}{d x^{2}}=-\frac{d E_{i}}{d x}=-\frac{q N_{i}}{\varepsilon_{s}} & \text { for } i=1, n \\
V_{i-1}\left(d_{i}\right)=V_{i}\left(d_{i}\right), E_{j-1}\left(d_{i}\right)=E_{i}\left(d_{i}\right) & \text { for } i=2, n \\
V_{1}\left(d_{1}\right)=V_{a}, V_{n}\left(d_{n+1}\right)=0 &
\end{array}
$$

where, $V_{i}$ and $E_{j}$ is the potential and the electric field in the ith layer, respectively. $\varepsilon_{\mathrm{s}}$ is the dielectric constant of the material and $q$ is the charge of an electron.

The first step is to integrate eq.(1) directly to give the following expressions.

$$
\begin{aligned}
& E_{j}=-q_{i} x+a_{i} \\
& v_{i}=-\frac{q_{i}}{2} x^{2}+a_{i} x+b_{i}
\end{aligned}
$$


where, $q_{i}=q N_{i} / \varepsilon_{S}$ and $a_{i}$ and $b_{i}$ are integral constants to be determined below.

Substituting eq.(4) into eq.(2) with $d_{1}=0$, we have the following expressions for the coefficients $a_{i}$ and $b_{i}$.

$$
\begin{array}{ll}
b_{1}=v_{a} & \\
b_{i}=b_{i-1}+\frac{q_{i-1}-q_{i}}{2} d_{i}^{2} & \text { for } i=2, n+1 \\
a_{n}=\frac{q_{n}}{2} d_{n+1}-\frac{b_{n}}{d_{n+1}} & \\
a_{i-1}=a_{i}+\left(a_{i-1}-q_{i}\right) d_{i} & \text { for } i=n, 2
\end{array}
$$

The last step is to substitute eq.(5) into eq.(4) to give the solution $E_{i}$ and $V_{i}$ in all the layers.

Three structures are considered here as follows.

\section{3-2-2. Buried p-i-n diode}

The first structure considered here is a "buried p-i-n" diade. Two lightly-doped layers were inserted in a simple $p-i-n$ diode. The object of such a structure was to reduce the peak field at the surface while keeping high field strength in most of the diode. An example structure and calculated field profile are shown in Fig.3-3. The assumed parameters are given in the caption. The maximum field occurs in a region away from the surface. This is the one of the structures already fabricated and measurements on it are discussed next. 


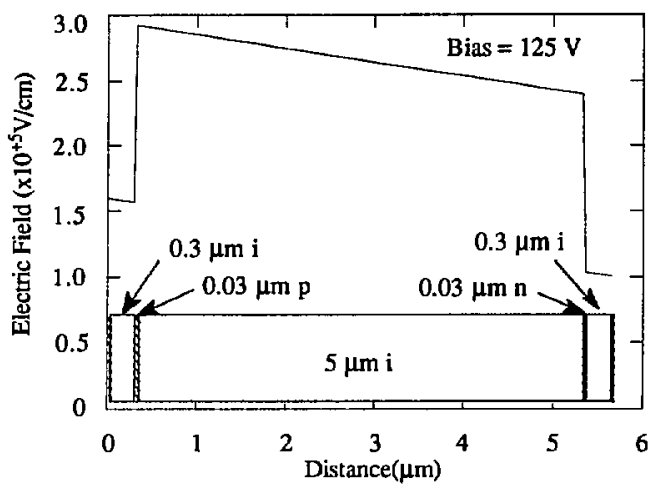

Fig.3-3 Structure and calculated field profile of a buried p-i-n diode. The heavily doped layers at both ends of the diode are not shown. The space density assumed in each layer is $7 \times 10^{+14} \mathrm{~cm}^{-3}$ in the $i$ layer and $\pm 3 \times 10^{+17} \mathrm{~cm}^{-3}$ in the lightly-doped layers. A bias of $125 \mathrm{~V}$ is assumed.

\section{3-2-2-1. Fabricatrion}

Intrinsic layers and lightly-doped layers were successively deposited at Xerox PARC to make a structure consisting of glass substrate / Cr / $0.03 \mu \mathrm{m} \mathrm{n}+/ 0.3 \mu \mathrm{mi} / 0.03 \mu \mathrm{m} \mathrm{n} / 5$ $\mu \mathrm{m}$ i $/ 0.03 \mu \mathrm{m} \mathrm{p} / 0.3 \mathrm{i} / 0.03 \mu \mathrm{m} \mathrm{p}^{+} / \mathrm{Cr}$ as shown schematically in Fig.3-3. Top Cr electrode is a disk $3 \mathrm{~mm}$ in diameter and it forms a parallel plate electrode together with the bottom $\mathrm{Cr}$ layer. The dopant gas concentrations for the lightly-doped layers are determined from the preparation conditions [7].

\section{3-2-2-2. Noise and I-V measurements}

The noise and current-voltage (I-V) characteristics of the buried p-i-n diodes were measured with a low noise charge-sensitive preamplifier and a quasi-Gaussian shaping amplifier ( $6.4 \mu \mathrm{sec}$ peaking time) connected. Figure 3-4 shows the measured noise and I- 
$\mathrm{V}$ characteristics of this $5.7 \mu \mathrm{m}$ thick buried $\mathrm{p}-\mathrm{i}-\mathrm{n}$ diode. The rapid noise increase of the buried $\mathrm{p}-\mathrm{i}-\mathrm{n}$ diodes occurs at an average field of about $50 \mathrm{~V} / \mu \mathrm{m}$ which is about three times larger than the case of the p-i-n diodes shown in Fig.3-1.

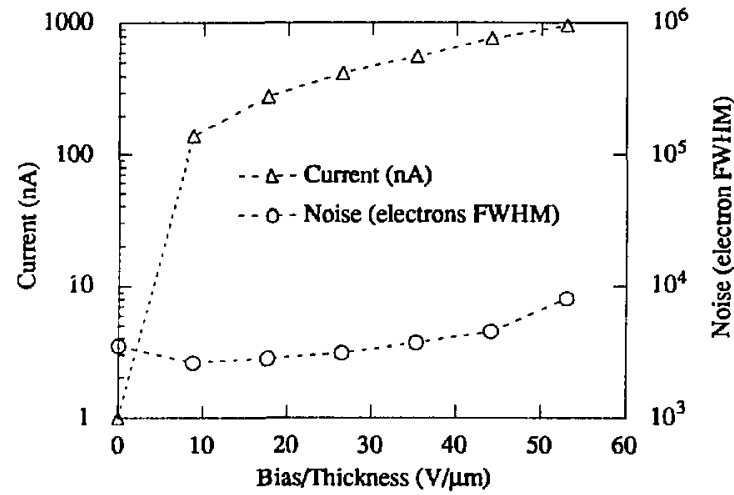

Fig.3-4 I-V and noise characteristics of the 5.7um thick buried p-i-n diode. The structure and the calculated field profile are shown in Fig.3-3.

The onset of this noise varied even among samples made from a single deposition run for both the conventional $\mathrm{p}-\mathrm{i}-\mathrm{n}$ diodes and these buried $\mathrm{p}-\mathrm{i}-\mathrm{n}$ diodes. Breakdown bias was defined somewhat arbitrary as the bias at which the noise exceeds three times the minimum noise level and a systematic study of this breakdown bias was conducted. Results are shown in Fig.3-5. The p-i-n diodes were all 16-17 $\mu \mathrm{m}$ thick with a $3 \mathrm{~mm}$ diameter disk $\mathrm{Cr}$ top electrode made from a single deposition run. This distribution for $\mathrm{p}-\mathrm{i}-\mathrm{n}$ diodes is a representative one for samples with different thickness. The buried $\mathrm{p}$-i-n diodes were the ones described above. This comparison clearly shows that the buried p-i-n structure is effective in suppressing the onset of the noise at high bias. 


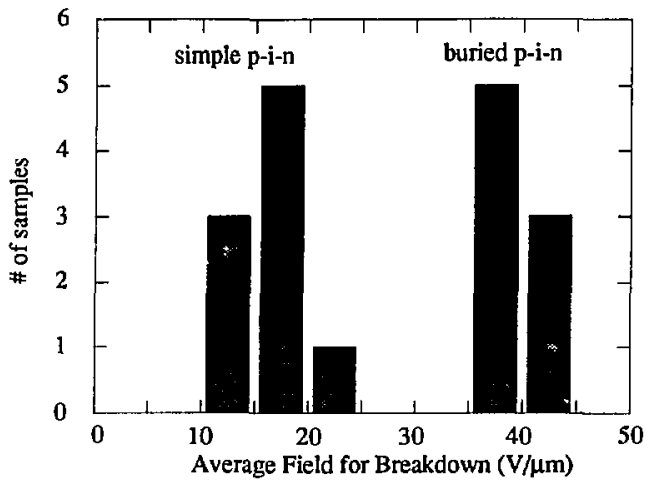

Fig.3-5 Comparison of the breakdown bias for the simple p-i-n diodes and the buried p-i-n diodes.

\section{3-2.2-3. Signal generation from $\alpha$ narticles}

It has been shown that a-Si:H radiation detectors suffer recombination loss when detecting high LET particles such as $\alpha$ particles $[2,8,9]$. With the higher field achieved by the buried $\mathrm{p}-\mathrm{i}-\mathrm{n}$ diodes, charge collection for $\alpha$ particle detection should be greatly improved. A model of charge collection and carrier recombination loss following an $\alpha$ particle transit through a fully-depleted a-Si:H diode and $\alpha$ particle detection by the biried $\mathrm{p}-\mathrm{i}-\mathrm{n}$ djodes are described. A simple theory which takes into accoun both "plasma erosion" and carrier recombination is developed and applied to $\alpha$ particle detection by the buried p-i-n diodes.

\section{Theory}

The plasma erosion process in crystalline silicon has been modeled by many authors who analyzed the fission fragment detection by surface-barrier type silicon detectors [10] and more recently by groups working on the bit-inversion error caused by energetic particle 
passage in digital integrated circuit chips [11]. The analysis described here follows the treatments by Seibt [10] for "end erosion" and Shur [11] for "surface erosion." A model of these two erosion mechanisms are schematically shown in Fig.3-6.

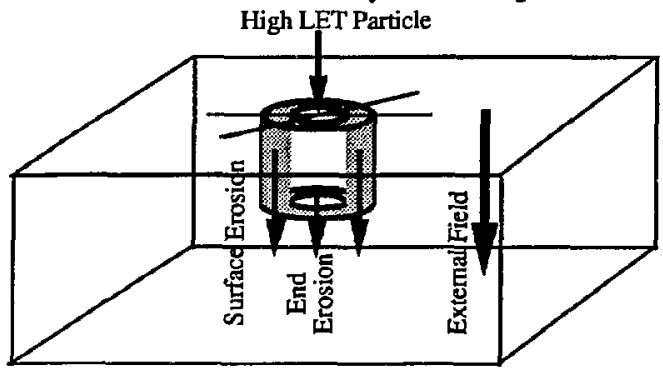

Fig.3-6 Plasma erosion model Two sources of erosion current are considered here.

The model by Shur assumes that the plasma column around the particle track is eroded by charge sweep-out at the column surface where the carrier density is at the level of the substrate doping and hence the external field is at its normal level. Carriers inside the plasma column have to move to the surface by ambipolar diffusion since the external field is shielded. The plasma density $P_{\mathrm{eh}}(\mathrm{r})$ is given by a parial solution of the diffusion equation in a cylindrical coordinate system;

$$
P_{\text {eh }}(r)=\frac{N_{o}}{4 \pi D r} \exp \left(-\frac{r^{2}}{4 D r}\right)
$$

where $D$ is the ambipolar diffusion coefficient, $t$ the time after the particle passage, and $N_{0}$ is the line plasma density defined as the normalization constant, i.e.,

$$
N_{0}=2 \pi \int_{0}^{\infty} P_{c h}(r) r d r
$$

At the surface of the plasma column $\left(r=r_{S}\right)$, the plasma density drops to $b N_{A}$ where $N_{A}$ is the substrate doping level and $b$ is a fitting parameter. The number of carriers available for drift is given by, 


$$
p_{s}=2 \pi \int_{r_{s}}^{\infty} P_{c h}(r) r d r=4 \pi b N_{A} D t
$$

The erosion current due to these carriers is $I=q_{s} \mu F$ where $q$ is the electron charge, $\mu$ is the carrier mobility and $F$ is the external field. The time derivative of the line density $N_{0}$ is given by,

$$
\frac{\mathrm{dN}_{\mathrm{o}}}{\mathrm{dt}}=-\frac{\mathrm{I}}{\mathrm{g} \mathrm{L}_{\mathrm{c}}}=-\frac{\mathrm{P}_{\mathrm{s}} \mu \mathrm{F}}{\mathrm{L}_{\mathrm{c}}}
$$

where $L_{c}$ is the length of the plasma column, i.e. the track length. Introducing dimensionless parameters $\mathrm{N}$ and $\mathrm{T}$ by the following expressions,

$$
N=\frac{N_{0}}{N_{00}}, \quad T=\frac{t}{t_{s}} \quad \text { where } t_{s}=\frac{N_{o 0}}{\pi D b N_{A}}
$$

where $N_{\infty}$ is the initial line density, and combining eq.(8) and (9), we obtain,

$$
\frac{d N}{d T}=-\frac{4 N_{o o} \mu F}{\pi D b N_{A} L_{c}} T
$$

Another possible source of the erosion current comes from the space charge limited current (SCLC) from the end of the plasma column initially located at the end of the track or the end of the detector layer whichever is shorter. The column length becomes shortened with time by the SCLC. Following the treatment by Seibt and using the notation by Shur given above, this contribution from the end of the column is expressed as,

$$
\frac{\mathrm{dN}}{\mathrm{dT}}=-\frac{8 \mu \varepsilon^{2} \mathrm{~F}^{3}}{\mathrm{D}\left(\mathrm{g} b \mathrm{~N}_{\mathrm{A}}\right)^{2} \mathrm{~L}_{\mathrm{c}}} \mathrm{T}
$$

where $\varepsilon$ is the dielectric constant of silicon.

When we evaluate the coefficient of $\mathrm{T}$ in eq.(11) and (12) using parameters for a-Si:H, it turns out that the contribution from the column end (eq.(12)) is the dominant mechanism for plasma erosion in the field range of our interest.

Next, the line carrier density change rate due to the recombination inside the plasma column is considered. This reconbination effect is negligible for crystalline silicon but 
significant for a-Si:H due to its low carrier mobility. Following, the treatment given by Adler [12], the time derivative of the volumetric carrier density $n$ is given by,

$$
\frac{\mathrm{dn}}{\mathrm{dt}}=-\frac{\mathrm{q} \mu}{\varepsilon} \mathrm{n}^{2}
$$

Converting eq.(13) into the equation for the line carrier density requires a detail knowledge of the radial carrier distribution. Instead, we simplify this process by assuming a uniform distribution inside the column radius $r_{s}$ so that $N_{0}=\pi r_{s}^{2} n$. Lising the dimensionless parameters defined in eq.(10) again, the recombination rate is given by,

$$
\frac{\mathrm{dN}}{\mathrm{dT}}=-\frac{\mathrm{q} \mu}{\varepsilon} \frac{\mathrm{N}_{\mathrm{Do}}^{2}}{\pi^{2} \mathrm{r}_{\mathrm{S}}^{2} \mathrm{D} \text { b N }} \mathrm{N}^{2}
$$

When we combine the effects of plasma erosion eq.(12) and recombination eq.(14), the line carrier density is given as a function of time from the solution of an ordinary differential equation of the form,

$$
\frac{d N}{d T}=-A T-B N^{2}
$$

This is the so-called Riccati differential equation and can be solved numerically.

If we define the plasma time $T_{p}$ as the time when the carrier density drops to zero, the number of the collected carriers and the recombined carriers per unit length of the detector are calculated respectively by,

$$
\begin{aligned}
& \mathrm{N}_{\text {coll }}=\int_{0}^{\mathrm{T}_{\mathrm{p}}} A \mathrm{~T} \mathrm{dT}=\frac{1}{2} A \mathrm{~T}_{\mathrm{p}}^{2} \\
& \mathrm{~N}_{\text {loss }}=\int_{0}^{\mathrm{T}_{\mathrm{p}}} \mathrm{B} \mathrm{N}^{2} \mathrm{dT}
\end{aligned}
$$

The collection efficiency eff. is given by,

$$
\text { eff. }=\frac{N_{\text {coll }}}{\mathbf{N}_{\text {coll }}+N_{\text {loss }}}=\frac{N_{\text {coll }}}{N_{\text {oo }}}
$$

\section{Measurements}

The $5.7 \mu \mathrm{m}$ and $15 \mu \mathrm{m}$ thick buried p-i-n diode:, were exposed to normally-incident 5.8 MeV $\propto$ particles from a Cf-249 source in vacuum. Signal size was recorded as a function 
of the bias across the unit diode thickness and is plotted in Fig.3-7. The $5.7 \mu \mathrm{m}$ buried p$\mathrm{i}-\mathrm{n}$ diodes are the one described above. The structure of the $15 \mu \mathrm{m}$ buried $\mathrm{p}$-i-n diodes is glass / Cr / $0.03 \mu \mathrm{m} \mathrm{n}+/ 0.3 \mu \mathrm{m}$ i / $0.06 \mu \mathrm{m} \mathrm{n} / 15 \mu \mathrm{m}$ i $/ 0.1 \mu \mathrm{m} \mathrm{p} / 0.3 \mu \mathrm{m}$ i $/ 0.03 \mu \mathrm{m}$ $\mathrm{p}+/ \mathrm{Cr}$. The contact size and the doping concentrations were all the same with those of the $5.7 \mu \mathrm{m}$ buried $\mathrm{p}-\mathrm{i}-\mathrm{n}$ diodes. The signal size from the conventional $\mathrm{p}-\mathrm{i}-\mathrm{n}$ diodes are also indicated by the box in the figure. In all diodes, the collected charges increase with bias and collection efficiency never reaches unity. This was attributed to recombination of carriers in the plasma column created by the $\alpha$ particle passage. This recombination loss is reduced by higher field because it erodes the plasma faster. The larger signal size from the buried $p$-i-n diodes cempared to the conventional p-i-n diodes indicates the presence of the high field.

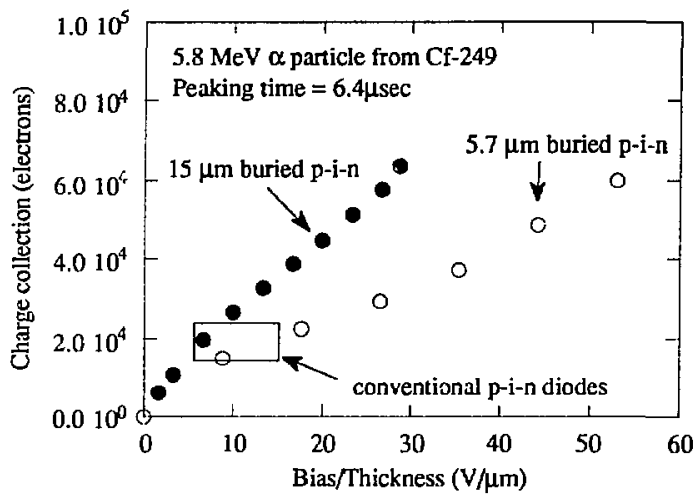

Fig.3-7 $\alpha$ particle detection with buried $\mathrm{p}$-i-n diodes

\section{Analysis}

Curve fitting $\alpha$ signal size as a function of bias is attempted with the additional data in high bias region provided by the buried $p-i-n$ diodes. The following parameter set was 
used for numericai analysis: $N_{00}=4.8 \times 10^{+8} \mathrm{~cm}^{-1}, \mu=1.0 \mathrm{~cm}^{2} / V \mathrm{sec}, D=5.2 \times 10^{-4} \mathrm{~cm}^{2} / \mathrm{s}$, $\mathrm{N}_{\mathrm{A}}=1 \times 10^{+15} \mathrm{~cm}^{-3}, \mathrm{~b}=1, \mathrm{~L}_{\mathrm{c}}=5 \times 10^{-4} \mathrm{~cm}, \varepsilon=1.06 \times 10^{-12} \mathrm{farad} / \mathrm{cm}, \mathrm{F}=1-6 \times 10^{+5} \mathrm{~V} / \mathrm{cm}$. The column radius $r_{s}$ in eq.(14) is the only fitting parameter used and the results with $r_{s}=$ $2.3 \mu \mathrm{m} \mathrm{g}$. $^{-}$the best fit as shown in Fig.3-9. Figure 3-8 shows N(T) for the same parameter set for six different fields i.e. $F=1-6 \times 10^{+5} \mathrm{~V} / \mathrm{cm}$. The characteristic time $t_{\mathrm{s}}$ defined in eq. $(10)$ is calculated to be $290 \mu \mathrm{sec}$. Therefore, the plasma times are approximately $80 \mathrm{~ns}, 35 \mathrm{~ns}$ and $15 \mathrm{~ns}$ for field strength of $1 \times 10^{+5}, 2 \times 10^{+5}, 5 \times 10^{+5} \mathrm{~V} / \mathrm{cm}$, respectively. The column radius introduced in eq.(14) should not be regarded as the real column size. The recombination rate strongly depends on the initial spatial distribution of carriers. The recombination takes place rapidly in the center of the column at the beginning of the charge collection process and subsides with time as the density decreases due to the erosion and recombination. The details of the spatial distribution were neglected for mathematical simplicity while maintaining the functional dependence on carrier density. The use of a more realistic value for the column radius $r_{s}$ such as $0.1 \mu \mathrm{m}$ (range of $\delta$ electrons)overestimates the recorabination loss. This may be explained by the presence of the "latent period" during which carriers are too hot to recombine with each other as discussed in [13]. In any event, the model is too crude to give further details. 


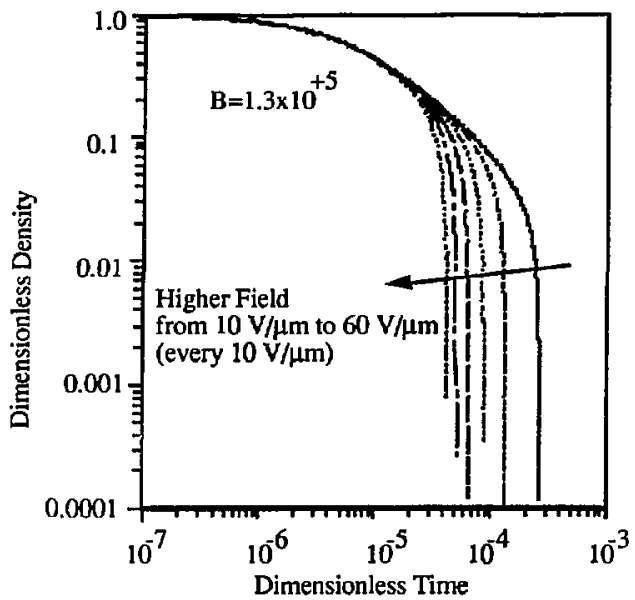

Fig.3-8 Carrier density inside plasma as a function of time for six different values of field. Both quantities are expressed in a dimensionless form defined in the text. The parameter $B$ is chosen to fit the charge collection data shown in the next figure.

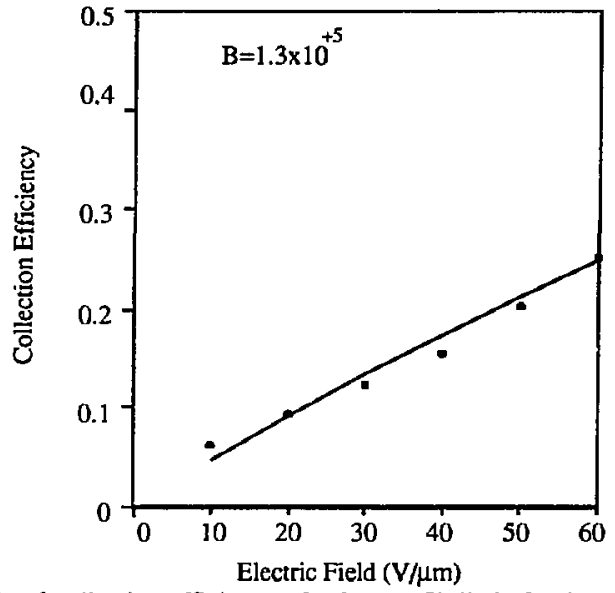

Fig.3-9 Calculated collection efficiency of a $5 \mu \mathrm{m}$ a-Si diode for detecting $6 \mathrm{MeV} \alpha$ particles. The parameter $B$ is chosen to fit the measured data for a buried p-i$\mathrm{n}$ diode which will be shown in the next chapter 


\section{3-2-3. $p-i-n$ diode with thick $p$ layer}

It has been reported that making the heavily doped $\mathrm{p}$ layer thicker in the $\mathrm{p}-\mathrm{i}-\mathrm{n}$ structure allows one to apply high bias on a-Si:H diodes [14]. A thick p layer works as a buffer layer to move the maximum field region away from the metal contact as in the case of the buried p-i-n diode. Assuming the same space charge densities with those in Fig.3-3, the field profile of a p-i-n diode with a thicker $\mathrm{p}$ is calculated and is shown in Fig.3-10. Because thicker layers are more conductive, a large edge current flows from the top electrode to the bottom electrode through these heavily doped layers, in effect, bypassing the diode. This edge current has to be reduced for low noise operation by etching the $p$ layers surrounding the contact electrode [15]. Aside from this additional process step, the thick $\mathrm{p}$ layer scheme seems simpler to implement than the buried $\mathrm{p}-\mathrm{i}-\mathrm{n}$ structure. The noise characteristics of these diodes were not described in ref.[14,15] and it was not clear how effective this structure is in suppressing the noise onset. Therefore, it was worthwhile to fabricate diodes with thick doped layers to measure the noise characteristics and variation in the bias of the noise onset and compare its performance with the buried p-i-n structure.

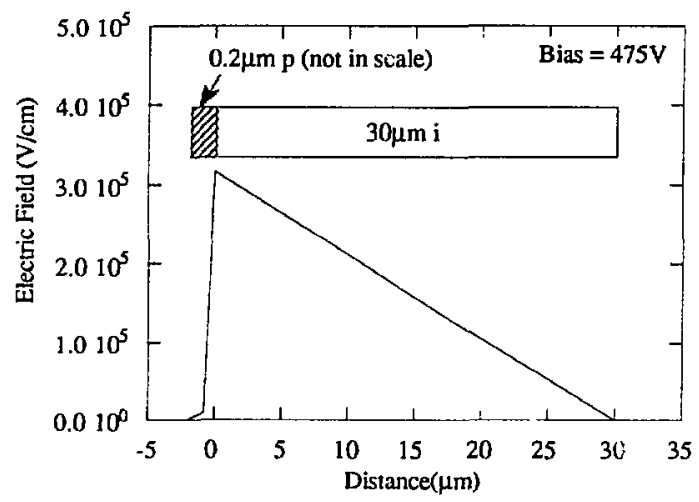


Fig.3-10 Calculated field profile for a p-i-n diode with a thick $p$ layer. The same space charge densities are assumed with those in Fig.3-3. The case of the critical bias for full depletion is shown.

\section{2-3.1. Fabrication}

Two sets of diodes with different doped layer thickness were fabricated at LBL. The structure of the first set is Glass $/ \mathrm{Cr} / 100 \AA \mathrm{n}+/ 8.7 \mu \mathrm{m} \mathrm{i} / 120 \AA \mathrm{p}+/ \mathrm{Cr}$. The top electrode is a $6 \mathrm{~mm}$ diameter $\mathrm{Cr}$ layer. The second set of $\mathrm{p}-\mathrm{i}-\mathrm{n}$ diodes has doped layers 10 times thicker than those of the first set. Only the deposition time of the doped layers were varied between these deposition runs. The deposition parameters are listed in Table 3-1.

Table 3-1. Deposition Conditions

\begin{tabular}{|l|l|l|l|l|l|l|l|}
\hline layer & $\begin{array}{l}\text { dep. time } \\
(\mathrm{min})\end{array}$ & $\begin{array}{l}\mathrm{T}_{\text {sub }} \\
\left({ }^{\circ} \mathrm{C}\right)\end{array}$ & $\begin{array}{l}\mathrm{RF} \\
(\mathrm{W})\end{array}$ & $\begin{array}{l}\mathrm{SiH}_{4} \\
(\mathrm{sccm})\end{array}$ & $\begin{array}{l}\text { doping gas } \\
(\text { sccm })\end{array}$ & $\begin{array}{l}\text { Pressure } \\
(\mathrm{mmT})\end{array}$ & $\begin{array}{l}\text { dep. rate } \\
(\mathrm{A} / \mathrm{min})\end{array}$ \\
\hline $\mathbf{n}$ & 1 or 10 & 320 & 4 & 40 & $10\left(\mathrm{PH}_{3}\right)$ & 680 & 100 \\
\hline $\mathrm{i}$ & 420 & 320 & 7 & 40 & $\mathrm{NA}$ & 680 & 210 \\
\hline $\mathrm{p}$ & 1 or 10 & 250 & 3.3 & 40 & $\begin{array}{l}5\left(\mathrm{~B}_{2} \mathrm{H}_{6}\right) \\
\left(00\left(\mathrm{CH}_{4}\right)\right.\end{array}$ & 800 & 120 \\
\hline
\end{tabular}

Note The substrate temperature is about $2 / 3$ of the setting shown above.

The gas cylinders for $\mathrm{PH}_{3}$ and $\mathrm{B}_{2} \mathrm{H}_{6}$ contain $5 \% \mathrm{PH}_{3}$ plus $95 \% \mathrm{SiH}_{4}$ and $5 \% \mathrm{~B}_{2} \mathrm{H}_{6}$ plus $95 \% \mathrm{He}$, respectively.

\section{3-2-3-2. Noise and $\mathrm{I}-\mathrm{V}$ measurements}

The noise and I-V characteristics were measured and results of a representative diode for each set of diodes are shown in Fig.3-11. Thick doped layers were effective in suppressing current at higher bias, indicating that the dominant contribution to current in this bias range is the contact injection. There was a wide variation in the noise behavior for both sets of diodes. Statistical study in Fig.3-12 confirmed that a higher tias can be applied to a diode with thick doped layers before the noise increase limits the detector operation. 


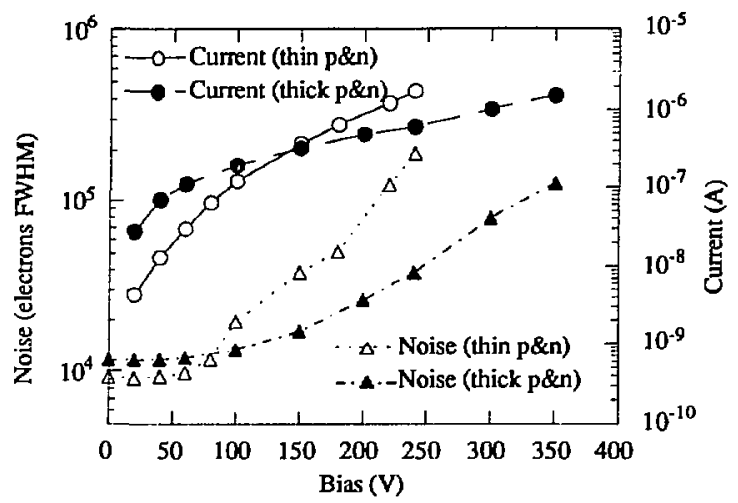

Fig.3-11 Noise and current-bias characteristics of a $8.7 \mu \mathrm{m}$ diodes with thin doped layers and thick doped layers.

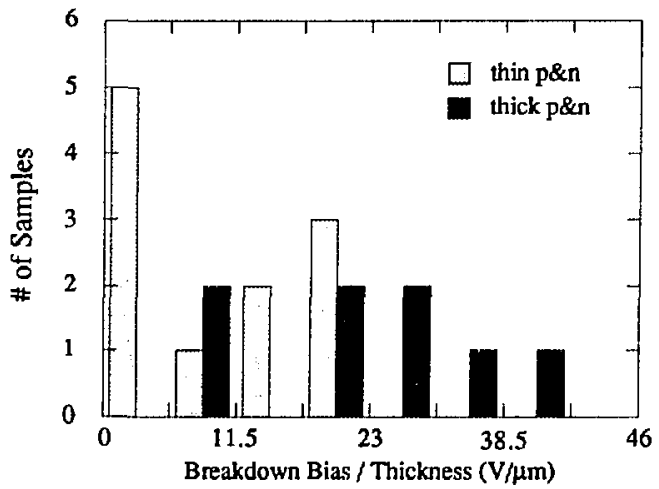

Fig.3-12 Breakdown bias for $8.7 \mu \mathrm{m}$-thick diodes with thin and thick doped layers. The top contact is a $6 \mathrm{~mm}$-diameter $500 \AA$-thick $\mathrm{Cr}$ layer for all the samples. 


\section{3-2-3-3. Discussions of relative merits}

The statistical studies about the breakdown bias as shown in Fig.3-5 and Fig.3-12 show that the buried $p-i-n$ structure is more effective in suppressing the noise onset, excluding the possibile differences in preparation conditions such as substrate cleaning procedures which are presumably equivalent. Therefore, it is worthwhile to insert all the doped layers into intrinsic layer, which can be done easily by a multi-chamber PECVD apparatus as shown in Chapter 1.

\section{3-2-4. Breakdown and ionized defect density}

Despite the success of the buried $p-i-n$ structure and the $p$-i-n diodes with thick doped layers, the argument of the breakdown mechanism is still not convincing because of the wide variation in the breakdown bias among samples made in a single deposition run as well as possible shortcomings for sample preparation. The following experiment was conducted in the hope of giving an additional evidence for tine near-surface breakdown hypothesis.

It is known in a-Si:H that in addition to the existing dangling bonds created during deposition, more defects (dangling bonds) are created by heating above a certain temperature when hydrogen atoms terminating unpaired Si bonds become mobile. These defects are located in the middle of the band gap and they release electrons under bias to become fixed positive space charges, which can be estimated by the Time of Flight (TOF) experiment [16]. Therefore, one can investigate the effect of the defect density on the breakdown bias by measuring the noise characteristics of a single diode before and after heating it.

A $12 \mu \mathrm{m}$ thick p-i-n diode made by GSI was heated at $300^{\circ} \mathrm{C}$ for 10 minutes to double this defect density $N_{d}^{*}[17]$. The noise characteristics and $N_{d}^{*}$ were monitored before and after the heating. The breakdown bias was decreased and the current was increased by the heating as shown in Fig.3-13. $\mathrm{N}_{\rfloor}^{*}$ measured by the TOF method was about $4 \times 10^{+14} \mathrm{~cm}^{-3}$ 
before and $8 \times 10^{+14} \mathrm{~cm}^{-3}$ after the heating. These numbers were smaller than what have been reported earlier [17] because the TOF measurements were done within 5 minutes after the bias application when the defects at the mid-gap states were still releasing electrons, although slowly. The field profiles before and after heating at each breakdown bias were calculated from each $\mathrm{N}_{\mathrm{d}}{ }^{*}$ and shown schematically in Fig.3-14. The maximum fields for both cases coincide approximately, offering evidence for the near-surface breakdown hypothesis.

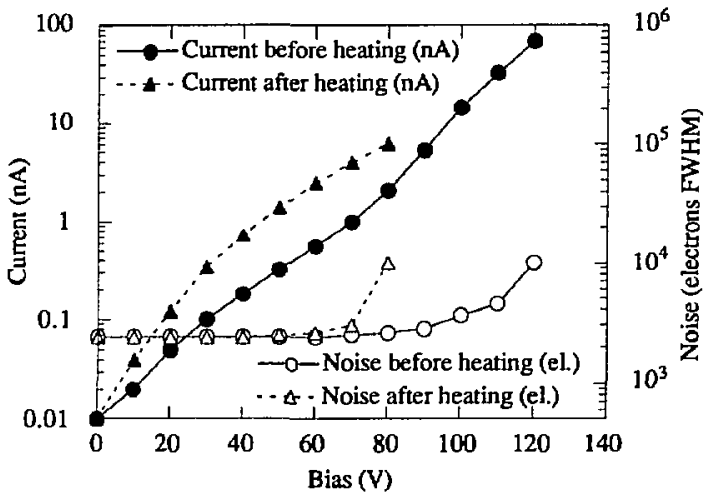

Fig.3-13 Noise and I-V characteristics of a $12 \mu \mathrm{m}$ thick p-i-n diode before and after heating at 300 " $\mathrm{C}$ for 10 minutes. $\mathrm{N}_{\mathrm{d}}^{*}$ measured by the TOF method was increased from $4 \times 10^{+14} \mathrm{~cm}^{-3}$ to $8 \times 10^{+14} \mathrm{~cm}^{-3}$ by this heating.

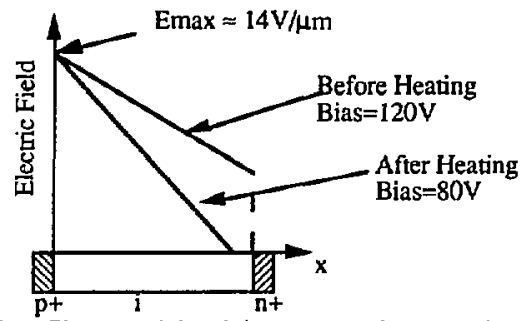

Fig.3-14 Field profiles at each breakdown bias before and after the heating. 


\section{3-2-5. p-i-n diode with buried $p$ layer}

Thicker a-Si:H p-i-n diodes require higher bias for full depletion since the field inside a diode has a gradient proportional to the ionized defect density in the intrinsic layer. This field gradient can be reversed by inserting a thin $\mathrm{p}$ layer which leaves negative space charges under depletion of free carriers [18]. A sample calculation of the field profile of this buried $p$ layer is shown in Fig.3-15. The thin $\mathrm{i}-\mathrm{p}$ structure is added to this $\mathrm{i}-\mathrm{p}-\mathrm{i}$ structure in order to decrease the field strength in the vicinity of the surface. The same space charge densities are assumed as those in Fig.3-3. The diode is over depleted at a bias of $1000 \mathrm{~V}$ and the maximum field strength is about $3.6 \times 10^{+5} \mathrm{~V} / \mathrm{cm}$. On the other hand, a bias of $1300 \mathrm{~V}$ is required to fully deplete a $50 \mu \mathrm{m}$ layer with space charge density of $7 \times 10^{+14} \mathrm{~cm}^{-3}$ and the peak field exceeds $5 \times 10^{+5} \mathrm{~V} / \mathrm{cm}$ which are calculated from eq.(14) in Chapter 2.

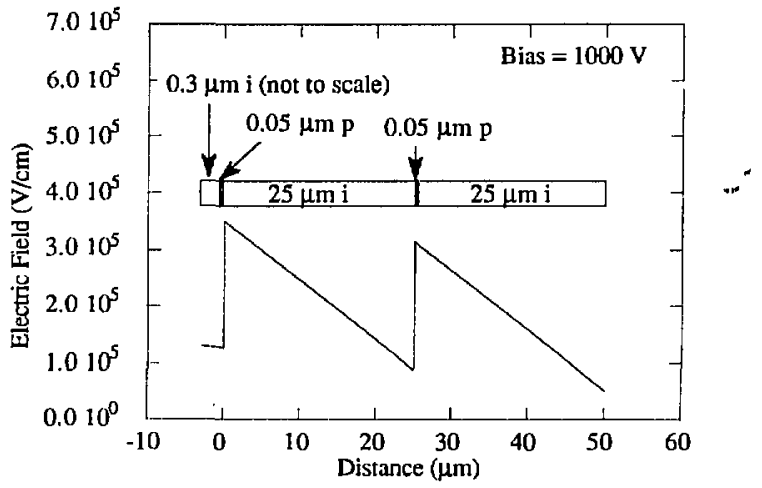

Fig.3-15 Calculated field profile for a buried $\mathrm{p}$ diode. The same space charge densities are assumed with those in Fig.3-3. 


\section{3-3. Tailoring by electrode shapes}

To improve charge collection efficiency of thick a-Si:H diodes, it is advantageous to have a uniform electric field profile. Using thick doped layers is not desirable for good efficiency because the carrier lifetime and mobility are strongly affected by doping [19]. Field profile can also be controlled by changing the shape of the electrodes. In a parallel plate configuration, the field changes linearly due to the fixed space charge in the intrinsic layer under bias. When one of the plates is made smaller than the other, the field close to the smaller electrode is enhanced roughly by the ratio of the electrode sizes. This is because the number of field lines landing on the two electrodes are the same. With both of these effects present, we can make the field profile more or less uniform or, if desired, can make a very non-uniform field profile, by the proper choice of electrode geometry and the right bias polarity.

\section{3-3-1. Field profile calculation for the cylindrical configuration}

Assume a coaxial structure of two cylinders of inner radius a and outer radius $\mathbf{R}$, sandwiching a dielectric material of a fixed constant space charge density of $\rho$ and dielectric constant $\varepsilon_{s}$. The Poisson equation is solved analytically with boundary conditions $V=V_{0}$ at $r=a$ and $V=0$ at $r=R$ to give the following expression for the field $E=-d V / d r$.

$$
E(r)=\frac{\rho}{2 E_{s}} r+\frac{V_{o}-\frac{\rho}{4 E_{s}}\left(R^{2}-a^{2}\right)}{r \ln \frac{R}{a}}
$$

With $\rho=0$, the field is inversely proportional to radius $r$. This is the direct consequence of the electrode shape, namely, all the field lines starting from the inner cylinder surface end up at the much larger outer cylinder surface, resulting to an enhanced field closer to the inner cylinder surface. For the case of intrinsic a-Si:H under bias, $\rho$ is positive. This enhances the field at large $r$ because the field lines starting from the fixed positive charge join the existing lines from the inner to outer radius. With both of these 
effect present, we can make the field profile more or less uniform or, if desired, make a very non-uniform field profile, by proper choices of these parameters.

\section{3-3-2. Microstrip electrode}

To illustrate the possibility of field compensation by the electrode geometry, a simple case of a $50 \mu \mathrm{m}$ thick a-Si i layer (the fixed space charge density $7 \times 10^{+14} \mathrm{~cm}^{-3}$ ) sandwiched by one continuous electrode and one discrete electrode is treated below. The code POISSON [20] was used to solve the resultant 2D-Poisson equation with suitable boundary conditions. Figure 3-16 shows the cross section of a unit cell of a diode with such electrodes. The bottom electrode is continuous and assumed to be biased at $-2000 \mathrm{~V}$ whereas the top electrode is discrete and grounded. The width and pitch of the top electrode are $4 \mu \mathrm{m}$ and $30 \mu \mathrm{m}$, respectively. The equipotential lines calculated by POISSON are shown every $200 \mathrm{~V}$ in Fig.3-16.

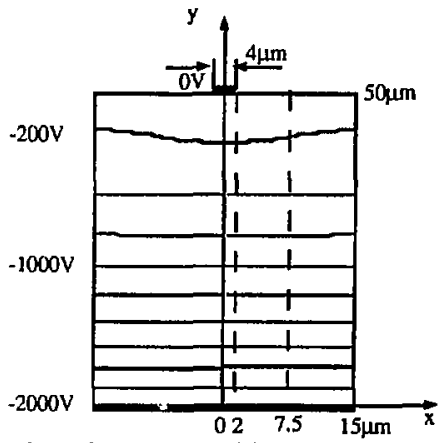

Fig.3-16 The cross-section of the diode with a continuous bottom electrode and a discrete top electrode. Equipotential lines are drawn every $200 \mathrm{~V}$ when the bottom electrode is biased at $-2000 \mathrm{~V}$.

The field strength along the lines $x=0,2,7.5,15 \mu \mathrm{m}$ in Fig.3-16 are plotted in Fig.17. The field drops linearly with the depth in the region close to the continuous electrode and it increases in the region close to the discrete electrode. The geometrical field enhancement compensates for the field drop due to the fixed space charge in the $\mathbf{i}$ layer to some extent. 
It is apparent that a low field region exists in the region near the top surface between the electrodes. This region should not have a significant eff $i$ t on charge collection process for the following reasons: (i) most of carriers generated in the bulk and drifting along field lines don't pass this region and (ii) as for the carriers created in this region, electrons, with their superior transport characteristics, have to drift only a small distance to the top electrode and hole contribution to signal is often lost anyway due to its low mobility and the limited collection time required by some applications. This structure has an additional advantage over the parallel plate configuration, namely, a reduced capacitance and, therefore, smaller delta noise.

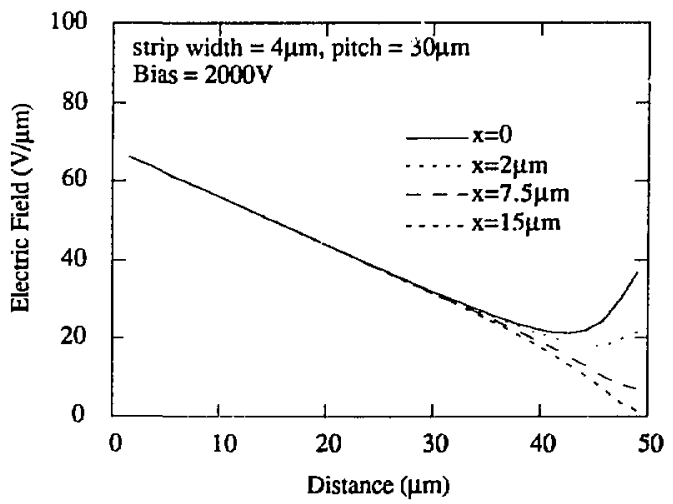

Fig.3-17 Calculated field profile of a diode with a continuous bottom electrode and a discrete top electrode. The field strength along lines $x=0,2,7.5$ and $15 \mu \mathrm{m}$ in Fig. 3-16 is shown here.

\section{3-3-3. Fabrication and measurements of microstrip electrode}

Two types of top $\mathrm{Cr}$ electrode patterns as shown in Fig.3-18 schematically were defined by standard photolithography techniques on a $28 \mu \mathrm{m}$ thick pin diode. The solid square portion was added to the strip pattern as shown in Fig.3-18 (b) for ease of making contact. 


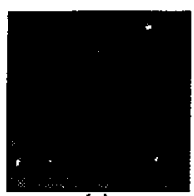

(a)

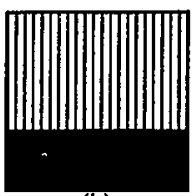

(b)

Fig.3-18 Schematic drawing of the two top electrode patterns: (a) solid square and (b) microstrip pattern with $2 \mu \mathrm{m}$ strip width and $10 \mu \mathrm{m}$ pitch attached to a $1 \times 3$ $\mathrm{mm}^{2}$ solid rectangule.

The structure of the diode was Glass / $\mathrm{Cr} / 30 \mathrm{~nm}-\mathrm{p}+/ 28 \mu \mathrm{m}-\mathrm{i} / 30 \mathrm{~nm}-\mathrm{n}+$. The bottom electrode was a plain $\mathrm{Cr}$ layer. The first top pattern was a $3 \times 3 \mathrm{~mm}^{2}$ solid square which forms a parallel plate electrode with the bottom $\mathrm{Cr}$ layer. The second type was a strip pattern with $2 \mu \mathrm{m}$ strip width and $10 \mu \mathrm{m}$ pitch. Based on the measured capacitance of these diodes, capacitance of a $100 \%$ microstrip pattern was estimated to be $65 \%$ of that of the solid pattern.

Noise and current-bias characteristics of these diodes were measured and the results are shown in Fig.3-19. The diode with a microstrip top contact showed a slight degradation in the noise behaviour although the currents were almost identical for both contacts. This suggests the presence of a higher local field. 


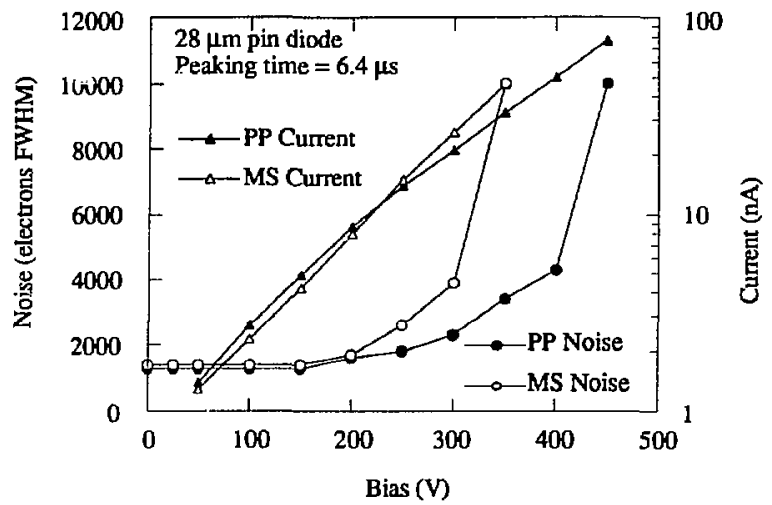

Fig.3-19 Noise and current-bias characteristics of $28 \mu \mathrm{m}$ pin diodes with the parallel plate electrodes (PP) and the mictostrip electrodes (MS).

Single carrier charge collection was measured by illuminating these diodes with $665 \mathrm{~nm}$ light pulses as in Chapter 2. The results are shown in Fig.3-20 The similar platau curves for these two types of electrodes indicate that the low field region in Fig.3-i6 does not affect the charge collection process in a diode with a microstrip electrode. 


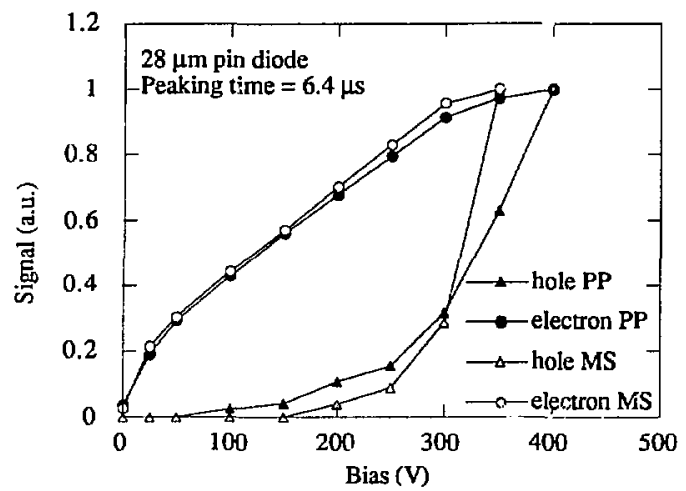

Fig. 3-20 Charge collection in the $28 \mu \mathrm{m}$ pin diodes with the parallel plate electrodes (PP) and the microstrip electrodes (MS).

\section{3-4. Conclusion}

Tailoring the field profile in a-Si:H radiation detectors as in the buried $\mathrm{p}-\mathrm{i}-\mathrm{n}$ structure was found to improve the charge collection process by allowing one to apply higher bias presumably through suppressing the near-surface breakdown phenomenon. It also gives a bias safety margin before breakdown for each element of a large area detector. A thicker $p$ layer adjacent to the metal contact also enabled higher bias operation. A microstrip electrode gave charge collection efficiency similar to that of a parallel electrode configuration with a smaller capacitance. 


\section{References}

1. V. Perez-Mendez, G. Cho, I. Fujieda, S.N. Kaplan, S. Qureshi and R.A. Street, "The Application of Thick Hydrogenated Amorphous Silicon Layers to Charged Particle and X-ray Detection," Proc. Mater. Res. Soc. Vol.149(1989)621.

2. B. Equer and A. Karar, "Effects of Primary Ionization in Amorphous Silicon Detectors," Nucl. Instr. Meth. A271(1988)574.

3. F. Goulding and D. Landis, "Signal Processiong for Semiconductor Detectors," IEEE Trans. Nucl. Sci., NS-29(1982)1125.

4. G. Cho, J. Drewery, I. Fujieda, S.N. Kaplan, S. Qureshi, V. Perez-Mendez and R.A. Street, "Measurements of $1 / \mathrm{f}$ Noise in a-Si:H pin Diodes and Thin Film Transistors", to be published in IEEE Trans. Nucl. Sci., NS-37(1990).

5. D.J. Rose, "Microplasma in Silicon," Physical Review 105(1957) 413.

6. R.A. Gibson, P.G. Le Comber and W.E. Spear, "The Characteristics of High Current Amorphous Silicon Diode," Appl. Phys. 21 (1980) 307.

7. M. Stutzmann, D.K. Biegelsen and R.A. Street, "Detailed Investigation of Doping in Hydrogenated Arnorphous Silicon and Germanium," Physical Review B35 (1987) 5666.

8. V. Perez-Mendez, S.N. Kaplan, W. Ward, S, Qureshi and R.A. Street, "Signal, Recombination Effects and Noise in Amorphous Silicon Detectors," Nucl. Instr. and Meth. A260(1987)195.

9. J. Dubeau, T. Pochet, A. Karar, L.A. Hamel, B. Equer, J.P. Martin, S.C. Gujrathi and A. Yelon, "Response of a-Si:H Detectors to Protons and Alphas," Proc. Mater. So... Vol.118(1988)439.

10. W.Seibt, K.E.Sundstrom and P.A.Tove, "Charge Collection in Silicon Detectors for Strongly lonizing Particles," Nucl. Instr. Meth. 113 (1973)317.

11 M.Shur, "Charge Collection by Drift during Single Particle Upset," IEFE Trans. Nucl. Sci. NS-33(1986)1140.

12. D.Adler, M.Siiver, A.Madan and W.Czubatyj, "Recombination Mecharisms in Amorphous Silicon-Based Alloys," J. Appl. Phys. 51 (1981)6429.

13. T.R.Oldham, "Recombination along the Tracks of Heavy Charged Particles in $\mathrm{SiO} 2$ Films," J. Appl. Phys. 57(1985)2695.

14. T. Pochet, J. DuBeau, L.A. Hamel, B. Equer and A. Karar, "Charge Collection in a-Si:H Particle Detectors," Proc. Mater. Res. Soc., Vol.149(1989)661.

15. T. Pochet, J. DuBeau, L.A. Hamel, B. Equer and A. Karar, "High Reverse Voltage Amorphous Silicon p-i-n Diodes," J. Appl. Phys. 68 (1990)1340. 
16. R.A. Street, "Measurements of Depletion Layers in Hydrogenated Amorphous Silicun," Physical Review, B27 (1983) 4924.

17. S. Qureshi, V. Perez-Mendez, S.N. Kaplan, I. Fujieda, G. Cho and R.A. Street, "Material Parameters in a Thick Hydrogenated Amorphous Silicon Detector and Their Effect on Signal Collection", Proc. Mater. Res. Soc., Vol.149(1989)649.

18. V. Perez-Mender, S.N. Kaplan, G. Cho, I. Fujieda, S. Qureshi, W. Ward and R.A. Street, "Hydrogenated Amorphous Silicon Pixel Detectors for Minimum Ionizing Particles," Nucl. Instr. Meth. A273(1988)127.

19 R.A. Street, J. Zesch, M.J. Thompson, "Effects of Doping on Transport and Deep Trapping in Hydrogenated Amorphous Silicon," A ppl. Phys. Lett. 43 (1983)672.

20 POISSON/SUPERFISH Los Alamos Accelerator Code, LA-UR-87-126, (1987). 


\section{Chapter 4. Scintillation light detection}

\section{4-1. Introduction}

In the indirect detection scheme, a scintillator converts incident radiations (charged particles, $\gamma$ rays or $X$-ray fluences) into visible light $(\lambda=300-700 \mathrm{~nm})$ which is then absorbed mostly at the surface of a thin $(1-2 \mu \mathrm{m})$ a-Si:H layer, creating electron and hole pairs. As discussed in Chapter 2, the drift motion of these carriers by the external field generates signal in the readout electronics which is designed to either count individual particles or integrate fluences, depending on each detector application.

Choice of a scintillator also depends on the kinds of radiation and photo-sensitive device and count rate capability required by each application. For a quick comparison, some properties of commonly-used scintillators are summarized in Table 4-1 [1,2].

Table 4-1 Properties of commonly-used scintillators

\begin{tabular}{|c|c|c|c|c|}
\hline Scintillator & $\begin{array}{c}\text { density } \\
\left(\mathrm{g} / \mathrm{cm}^{3}\right)\end{array}$ & $\begin{array}{c}\text { emission peak } \\
(\mathrm{nm})\end{array}$ & $\begin{array}{c}\text { decay const } \\
(\mu \mathrm{s})\end{array}$ & $\begin{array}{c}\text { light yield } \\
(\text { photons } / \mathrm{MeV})\end{array}$ \\
\hline $\mathrm{Nal}(\mathrm{Tl})$ & 3.67 & 415 & 0.23 & 38000 \\
\hline $\mathrm{BGO}$ & 7.13 & 480 & 0.30 & 8200 \\
\hline $\mathrm{Cs}(\mathrm{Tl})$ & 4.51 & 550 & 1.0 & 53000 \\
\hline $\mathrm{Csl}(\mathrm{Na})$ & 4.51 & 420 & 0.63 & 38000 \\
\hline $\mathrm{CsI}$ pure & 4.51 & 305 & $0.01-0.03$ & 17000 \\
\hline $\mathrm{CdWO}_{4}$ & 7.90 & 540 & 5.0 & 15000 \\
\hline $\mathrm{CaWO}_{4}$ & 6.1 & 430 & 6.0 & 8000 \\
\hline $\mathrm{CaF}_{2}(\mathrm{Eu})$ & 3.19 & 435 & 0.94 & 24000 \\
\hline $\mathrm{GSO}$ & 6.71 & 430 & $0.06-0.6$ & 14000 \\
\hline $\mathrm{NE} 110$ & 1.1 & $350-450$ & $0.002-0.02$ & 10000 \\
\hline $\mathrm{NE} 102 \mathrm{~A}$ & 1.1 & $350-450$ & $0.002-0.02$ & 10000 \\
\hline
\end{tabular}

High density is desirable for detecting $\chi$-rays or $\gamma$ rays to ensure good detection efficiency. Emitted photons form some continuous spectrum and this shape is an important factor for selecting a photon-sensitive device such as photomultiplier and photodiode. Scintillation occurs immediately after absorbing radiation and its light emission decays exponentially with time. It is often sufficient to treat this decay as a single 
component exponential in most cases. A shorter decay constant is preferted for applications requiring high count rate capability. Since there ir a linear relation between the number of emitted photons and the energy deposited by radiation in most cases, it is common to express a light yield of a scintillator in terms of number of photons per unit energy absorbed. High light yield is desirable for larger output, especially for coupling to a photon detector without internal multiplication mechanisms.

Another important factor to consider especially for imaging applications is the light spread inside a scintillator which degrades the spatial resolution. A scintillation faceplate [3] as shown schematically in Fig.4-1 has been developed to cope with this problem and become available from several manufactures [4]. Glass fibers containing rare-earth elements are fused and drawn to make a few $\mu \mathrm{m}$ columnar structure. Scintillation light is confined in each fiber thus the degradation of spatial resolution caused by the light spread is avoided. Although the light yield of the rare-earth element scintillation faceplates is not high enough to detect single minimum ionizing particles, it is adequate for detecting intense fluences in the curient-mode operation.

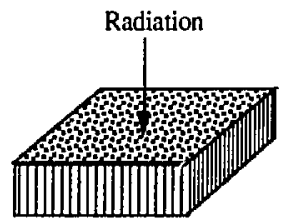

Fig.4-1 Scintillation faceplate. Glass tubing containing rare-earth elements is stacked, drawn and cut. Scintillation light is confined in a narrow region parallel to the plane normal.

Alternatively, a similar coluninar structure can be made by $\mathrm{Csl}(\mathrm{Na})$ evaporation as used in the manufacturing process of an Image Intensifier $[5,6]$. Vacuum evaporated CsI forms columns parallel to the evaporation direction. The column diameter can be controlled by the sut.strate temperature. These columns form natural light pipes that function similarly to the 
optical fiber described earlier, confining the light to produce a sharper image output at the phosphor-detector interface. CsI(Na) layers $300 \mu \mathrm{m}$ thick with $5-10 \mu \mathrm{m}$ column diameter are routinely made by manufacturers of $\mathrm{X}$-ray image intensifiers for medical imaging [7].

Signal size in number of electron-hole pairs for the indirect detection scheme can be expressed as follows.

$$
S=\left(1-L_{o p 1}\right) \cdot Q E \cdot T Y \cdot E_{d}
$$

where,

$$
\begin{aligned}
& T Y=\int Y(\lambda) d \lambda \\
& Q E=\frac{\int \pi(\lambda) Y(\lambda) d \lambda}{T Y}
\end{aligned}
$$

Here, $E_{d}$ is the energy deposited in the scintillator. A certain number of visible light photons are created by radiation (light yield per unit energy absorbed is denoted as $Y(\lambda)$ where $\lambda$ is the wavelength), propagated to a photodiode through an optical coupling (if any) with some loss factor denoted as $\left(1-\mathrm{L}_{\text {opt }}\right)$ and electron-hole pairs are created in a photodiode with quantum efficiency $\eta(\lambda)$. Therefore, two correction factors must be known to determine an absolute light yield in terms of number of photons per absorbed energy, namely, the quantum efficiency factor $\mathrm{QE}$ and the optical loss factor (1- $\left.\mathrm{L}_{\mathrm{opt}}\right)$. The former can be obtained from the known emission spectra and a quantum efficiency curve of the photodiode. Solid curves in Fig.4-2 show the normalized emission intensity $Y(\lambda)$ for pure $\operatorname{CsI}, \operatorname{CsI}(\mathrm{Na})$ and $\operatorname{CsI}(\mathrm{TI})[8]$ and dotted curves are the quantum efficiencies $\eta(\lambda)$ of a standard photocathode for photomultipliers (Sb-Cs) [9], a crystalline Si photodiode (Hamamatsu S1723-04) [9] and a-Si:H photodiode in ref.[10]. Integration of the product $\eta(\lambda) \mathrm{Y}(\lambda)$ is $52 \%$ of the total light yield of $\mathrm{CsI}(\mathrm{Na})$ for $\mathrm{S} 1723-04$. The corresponding value for $\mathrm{CsI}(\mathrm{Tl})$ is $70 \%$. The quantum efficiency of this a-Si:H photodiode is superior to that of \$1723-04 in the blue to yellow region. The second correction factor, the optical loss factor can be obtained if we use a scintillator whose light yield is accurately known. 
In the following discussions, the observed signal size from a photodiode will be given in terms of electrons rather than attempting to unfold the observed signals by these two correction factors.

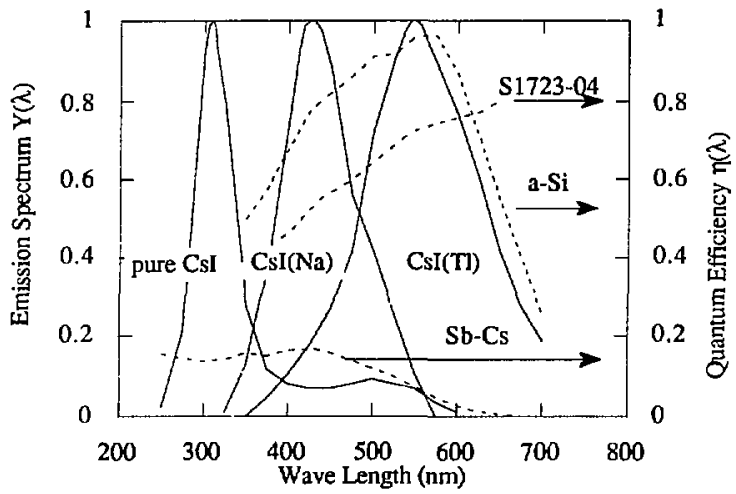

Fig.4-2 Noemalized emission spectra of pure CsI, CsI(Na) and CsI(Tl) and quantum efficiencies of a photocathode (Sb-Cs), the Hamamatsu photodiode S1723-04 and the a-Si:H photodiode in ref.[10].

$\operatorname{CsI}(\mathrm{Na})$ is used for image intensifier application due to its good emission spectrum matching to the photocathode sensitivity as can be seen in Fig.4-2. However, the following advantages over $\mathrm{CsI}(\mathrm{Na})$ are expected from $\mathrm{CsI}(\mathrm{Tl})$ for coupling to a photodiode; (a) better emission spectrum matching to a photodiode quantum efficiency, (b) larger light yield and (c) better stability in air, although it has a disadvantage of a slightly slower scintillation decay.

In this chapter, the evaporation process and some characteristics of evaporated CsI layers such as signal yield, columnar structure and radiation damage will be described first. Detection of $X$-ray pulses and single $\beta$ particles will be demonstrated with an evaporated CsI layer coupled to a photodiode. A monolithic type detector will be constructed and used to detect X-ray pulses. 


\section{4-2. CsI evaporation}

The vacuum evaporation setup used is shown schematically in Fig.4-3. The imporant parameters are the substrate temperature and the boat temperature [6]. The higher substrate temperature tends to give larger fiber structure and better adhesion to smooth glass surfaces. Lower substrate temperature tends to give finer columnar structure. The boat temperature determines the evaporation rate.

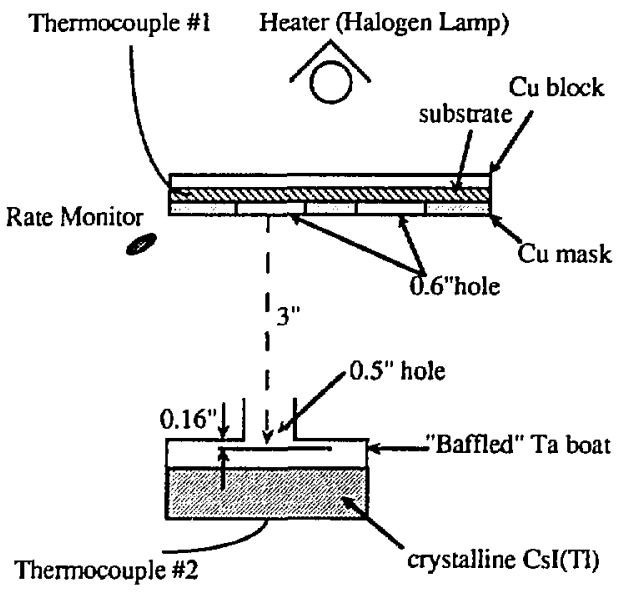

Fig.4-3 The vacuum evaporation setup for CsI faceplate fabrication.

Using the setup shown in Fig.4-3, $\operatorname{Csl}(\mathrm{Na})$ and $\mathrm{CsI(Tl)}$ layers wete evaporated on glass substrates. The glass surface had to be roughened to improve CsI adhesion for evaporation on low temperature substrates $\left(5300^{\circ} \mathrm{C}\right)$. Thickness of an evaporated layer was determined from the measured weight increase of the substrate and the known area size, assuming that the density of CsI is the same as crystal CsI $\left(4.51 \mathrm{gm} / \mathrm{cm}^{3}\right)$. It was found later that the physical thickness is $10-20 \%$ larger than the calculated value from the weight increase, indicating that the density of an evaporated layer is $10-20 \%$ smaller thatn the crystal CsI. However, quoting thickness determined from the weight measurement is 
more appropriate for $\mathrm{X}$-ray detection than the physical thickness because the mass thickness (physical thickness mutiplied by density) controls X-ray absorption. Therefore, thickness calculated from the weight measurement is quoted for an evaporated layer in the following sections.

First, CsI(Na) layers were evaporated on roughened glass substrates from a powder nixture of $99 \%$ weight CsI plus $1 \%$ NaI. A small amount of powder mixture was put into the Ta boat and was all evaporated for accurate thickness control. The evaporation time was usually about $20 \mathrm{~min}$ for a $300 \mu \mathrm{m}$ thick CsI(Na) layer. The glass substrate was initially heated to $100^{\circ} \mathrm{C}$ but its temperature increased to $140^{\circ} \mathrm{C}$ by the end of a run due to radiation heating from the Ta boat. The boat temperature also increased by up to $30^{\circ} \mathrm{C}$ at the end of a run as the material left the boat.

Second, CsI(Tl) layers were evaporated from a single crystal CsI(TI) supplied by Engelhard Corp. with the same evaporation conditions. Evaporation from a powder mixture was not attempted because TIl is highly toxic and the vapor pressure of Tll at the melting point of CsI is much higher than that of CsI [5]. For the CsI(T7), the evaluation rate was varied to improve the light yield. This will be described later.

\section{4-3. X-ray test setup and CsI signal yield measurement}

\section{4-3-1. X-ray source and test setup deseription}

The X-ray test set up system shown in Fig.4-4 was installed and used for the experiments described in this chapter. The grid potential of the $\mathrm{X}$-ray tube was controlled by a pulse generator and a Control Box to generate desired $\mathrm{X}$-ray pulsing conditions (pulse width from 1 ss to DC, repetition rate up to $1 \mathrm{kHz}$, intensity up to $1 \mathrm{~mA}$ of current level). The generated $\mathrm{X}$-ray has a continuous energy spectrum whose maximum energy corresponds to the bias applied by the HV Power Supply. Thin brass and Al and brass sheets inserted between the tube and the Shield Box absorbed low energy portion of the $\mathrm{X}$ - 
ray spectrum, resulting in a narrower spectrum. This narrow spectrum was treated as monochromatic. The error associated with this approximation is analyzed in sec. 4-3-3.

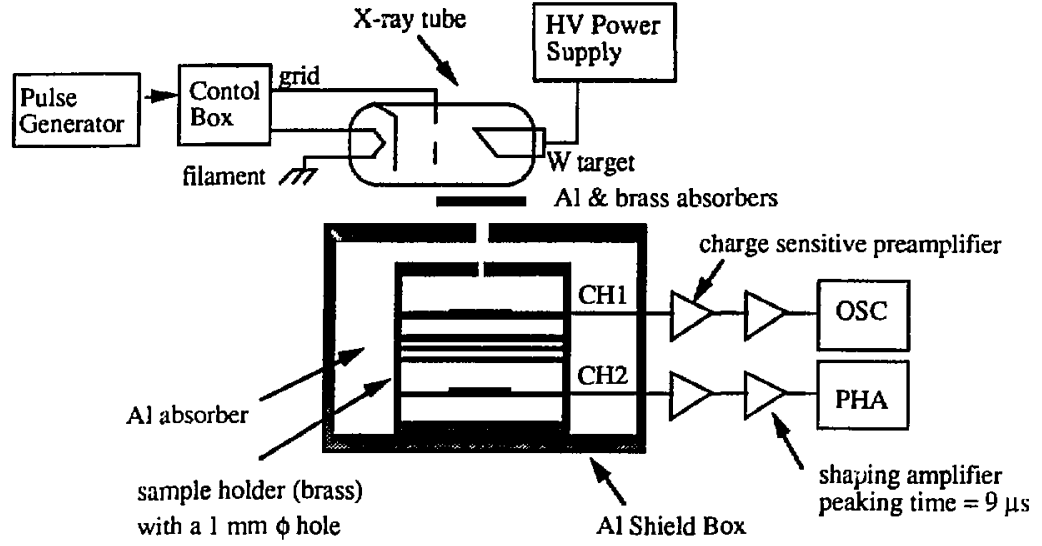

Fig.4-4 Schematic drawing of the $X$-ray system setup.

In the Shield Box, there are two sets of radiation detectors and their readout electronics (charge sensitive preamplifiers and shaping amplifiers). $\mathrm{CH} 1$ is a standard crystalline $\mathrm{Si}$ radiation detector without any backing materials. $\mathrm{CH} 1$ is used to monitor and control the total X-ray energy dumped into detectors placed in $\mathrm{CH} 2$. $\mathrm{CH} 2$ is the detector of interest, namely, a detector consisting of an evaporated CsI layer and a photodiode. Additional Al absorber was placed between $\mathrm{CH}$ ] and $\mathrm{CH} 2$ to determine the average energy of the X-ray spectrum during the calibration process which will be described next.

\section{4-3-2. $X$-ray source calibration and CsI signal yield measurement}

First, an evaporated CsI(T) layer $461 \mu \mathrm{m}$ thick coupled to a crystalline Si $\mathrm{p}-\mathrm{i}-\mathrm{n}$ photodiode (Hamamatsu S1723-04) was placed in $\mathrm{CH} 2$. This photodiode was extensively used in this chapter because; (a) it was easy to collect all the scattered scintillation light due 
to its fairly large size ( $1 \mathrm{~cm} \times 1 \mathrm{~cm}$ area), (b) it had a small capacitance $(50 \mathrm{pF})$ for low noise opcration, (c) its quantum efficiency was calibrated by the manufacturer and (d) it permits testing of phosphors separate from a-Si:H photodiode characteristics.

$X$-ray pulses were generated for three different settings of the HV Power Supply (50 $\mathrm{kV}, 30 \mathrm{kV}$ and $20 \mathrm{kV}$ ). The pulse width was set to $1.5 \mu \mathrm{s}, 3 \mu \mathrm{s}$ and $5 \mu \mathrm{s}$ for $50 \mathrm{kV}, 30 \mathrm{kV}$ and $20 \mathrm{kV} \mathrm{X}$-rays, respectively, to produce a large signal for easy detection but short enough to avoid the ballistic deficit, a signal loss when the signal ge:1eration takes longer than the peaking time (the peaking time was set to $9 \mu \mathrm{s}$ ). The thickness of the Al absorber between $\mathrm{CH} 1$ and $\mathrm{CH} 2$ was varied for each case to obtain the absorption curves shown in Fig.4-5. These three curves were fitted by exponential curves to give effective linear attenuation coefficients of $\mathrm{Al}$, denoted as $\mu_{\mathrm{Al}}$, for these $\mathrm{X}$-ray spectra.

Based on the attenuation coefficients given by this curve fitting $\mu_{\mathrm{Al}}$, the average energies of the $\mathrm{X}$-ray spectrum coming out of the crystalline $\mathrm{Si}$ detector in $\mathrm{CH} 1$, denoted as $E_{a}$, were determined from the X-ray cross section table for Al. The coefficients for Si and CsI at these energies are given from the cross section table and the Harshaw (Engelhard) catalogue [2]. These are summarized in Table 4-2. 


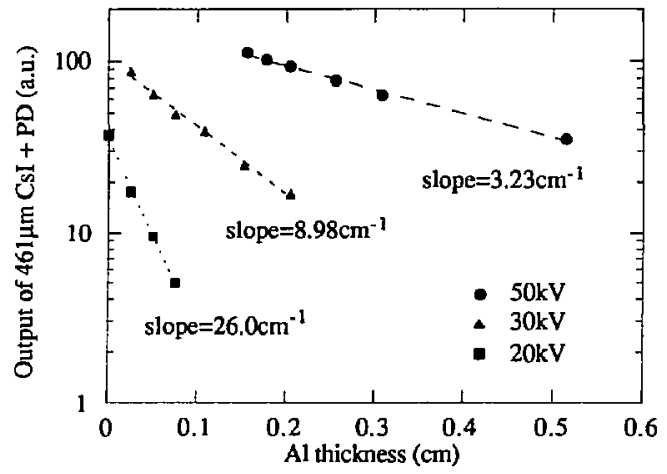

Fig.4-5 Absorption curve of $\mathrm{Al}$ for three kinds of $\mathrm{X}$-ray spectra.

Table 4-2 Average energies and attenuation coefficients

\begin{tabular}{|ll|c|c|c|}
\hline HV Power Supply & {$[\mathrm{kV}]$} & 50 & 30 & 20 \\
\hline pulse width & {$[\mu \mathrm{s}]$} & 1.5 & 3.0 & 5.0 \\
\hline$\mu_{\mathrm{A}]}$ & {$\left[\mathrm{cm}^{-1}\right]$} & 3.23 & 8.98 & 26.0 \\
\hline $\mathrm{E}_{\mathrm{a}}$ & {$[\mathrm{keV}]$} & 30.5 & 20.8 & 14.0 \\
\hline$\mu_{\mathrm{Si}}$ & {$\left[\mathrm{cm}^{-1}\right]$} & 3.25 & 9.65 & 29.7 \\
\hline$\mu_{\mathrm{CSl}}$ & {$\left[\mathrm{cm}^{-1}\right]$} & 45.3 & 119 & 322 \\
\hline
\end{tabular}

Next, a $210-\mu \mathrm{m}$-thick crystalline-Si radiation detector was placed in $\mathrm{CH} 2$ and the thickness of the Al absorber was fixed for each HV setting. The same X-ray pulses were generated as before and the output of the Si detector was recorded. Table 4-3 gives the calculated values of energy deposited in the detector. The energy deposited in the $210 \mu \mathrm{m}$ Si layer by a single X-ray pulse, denoted as $\mathrm{E}_{d} \mathrm{Si}$, is given by the output of $\mathrm{CH} 2$ (expressed in terms of electrons) multiplied by the average energy needed to create an electron-hole pair in crystalline $\mathrm{Si}(3.6 \mathrm{eV})$. The energy deposited in a $461 \mu \mathrm{m}$ CsI layer by the same 
single $\mathrm{X}$-ray pulse, denoted as $\mathrm{E}_{\mathrm{d}} \mathrm{CsI}$, is obtained by multiplying $\mathrm{E}_{\mathrm{d}} \mathrm{Si}$ by the ratio of the absorption probabilities.

Table 4-3. Energy deposition in Si and CSI

\begin{tabular}{|ll|c|c|c|}
\hline HV Power Supply & {$[\mathrm{kV}]$} & 50 & 30 & 20 \\
\hline Al thickness & {$[\mathrm{mm}]$} & 2.05 & 1.02 & 0.254 \\
\hline $\mathrm{E}_{\mathrm{d}}$ Si & {$[\mathrm{MeV}]$} & 0.758 & 0.855 & 0.984 \\
\hline $\mathrm{E}_{\mathrm{d}}$ Csi & {$[\mathrm{MeV}]$} & 10.1 & 4.96 & 2.11 \\
\hline
\end{tabular}

Finally, a CsI(Tl) layer $461 \mu \mathrm{m}$ thick coupled to the Hamamatsu photodiode (S1723-04) was placed in $\mathrm{CH} 2$ and was exposed to the same $\mathrm{X}$-ray pulses. The Hamamatsu photodiode is about $200 \mu \mathrm{m}$ thick and sensitive to the $X$-rays. Therefore, the signal from the photodiode can contain a contribution from electron-hole pairs created directly by the $\mathrm{X}$ ray if the $\mathrm{X}$-ray absorption in the scintillator is not $100 \%$. This direct signal contribution can be measured by inserting a thin opaque sheet between the photodiode and a scintillator. The real scintillation signal was obtained by subtracting this direct contribution from the observed signal without the opaque sheet. The photodiode output (expressed in terms of electrons) divided by the energy deposition $\mathrm{E}_{d}{ }^{\mathrm{CsI}}$ gives the signal yield of this CsI and photodiode coupling. Table 4-4 lists these values. The CsI signal yields expressed in number of electrons/ $\mathrm{MeV}$ measured by these three different $\mathrm{X}$-ray spectra roughly agree. When the error analysis described in the next section is taken into account, the agreement is even better.

Table 4-4 CsI signal yield

\begin{tabular}{|ll|c|c|c|}
\hline HV Power Supply & $\mid \mathrm{kV}]$ & 50 & 30 & 20 \\
\hline CsI Output & {$[\mathrm{e}]$} & $2.82 \times 10^{+5}$ & $1.15 \times 10^{+5}$ & $5.39 \times 10^{+4}$ \\
\hline CsIYield & {$[\mathrm{e} / \mathrm{MeV}]$} & $2.79 \times 10^{+4}$ & $2.48 \times 10^{+4}$ & $2.55 \times 10^{+4}$ \\
\hline Conrection Factor & & 1.18 & 1.07 & 1.08 \\
\hline Cor. CsI Yield & {$[\mathrm{e} / \mathrm{MeV}]$} & $2.37 \times 10^{+4}$ & $2.32 \times 10^{+4}$ & $2.36 \times 10^{+4}$ \\
\hline
\end{tabular}




\section{4-3-3. Error analysis}

The energy deposition in Csl is estimated from the measured energy deposited in a $\mathrm{Si}$ detector by assuming a continuous $\mathrm{X}$-ray spectrum as a monochromatic spectrum. The error involved in this average energy analysis is treated here. First, assume some energy dependency on the initial $X$-ray spectrum $I_{0}(E)$. After passing through Al absorter of thickness $t_{A l}$, the spectrum becomes,

$$
\mathrm{I}\left(\mathrm{E}, \mathrm{t}_{\mathrm{Al}}\right)=\mathrm{I}_{0}(\mathrm{E}) \exp \left(-\mu_{\mathrm{Al}}(\mathrm{E}) \mathrm{t}_{\mathrm{Al}}\right)
$$

The energy deposited in a Si layer of thickness $\mathrm{t}_{\mathrm{Si}}$ is written as,

$$
E_{d}^{S^{i}}=\int_{0}^{F_{i}} I\left(E, t_{A]}\right) E\left[1-\exp \left(-\mu_{S i}(E) t_{S i}\right)\right] d E
$$

The energy deposited in a Csl layer of thickness tcsl is given by,

$$
E_{d}^{C s I}=\int_{0}^{F_{0}} I\left(E, t_{A}\right) E\left[1-\exp \left(-\mu_{C s I}(E) t_{C s l}\right)\right] d E
$$

Second, a simple delta function is assumed for a presumably narrow spectrum in the average energy analysis. The initial spectrum is replaced by,

$$
I_{0}(E)=A \delta(E-\bar{E})
$$

Eq.(5) and (6) are then rewritten as,

$$
\begin{aligned}
& E_{d}^{S i}=A \exp \left[-\mu_{A}(\bar{E}) t_{A d}\right]\left[1-\exp \left(\mu_{S i}(\bar{E}) t_{S i}\right)\right] \\
& E_{d}^{C s I}=A \exp \left[-\mu_{A}(\bar{E}) t_{A d}\right]\left[1-\exp \left(\mu_{C s}(\bar{E}) t_{C S I}\right)\right]
\end{aligned}
$$

Therefore, the ratio of energy depositions in two layers is simply the ratio of the absorption probability in each layer at the average energy.

What determines the error involved in replacing eq.(5) and (6) by eq.(8) and (9), respectively? The obvious factor is the "narrowness" of the spectrum. If the spectrum is as sharp as the delta function, eq.(8) and (9) are exact. If not, the error depends on the attenuation coefficients of the materials in addition to the sharpness of the spectrum. If the coefficients are similar in both materials, the whole spectrum is equally absorbed and the error is small. If there is a big difference in coefficients as in the case of Si and CsI, the 
whole spectrum is not equally absorbed. CsI $460 \mu \mathrm{m}$ thick absorbs almost everything except a small portion of the high energy tail in a $50 \mathrm{kV} \mathrm{X}$-ray spectrum. Si $210 \mu \mathrm{m}$ thick ubsorbs only a small fraction of the spectrum and the energy absorbed is mostly due to the lower energy portion of the spectrum.

To express the error involved in the average energy analysis, a correction factor is defined as the ratio of the exact estimate of $E_{d} C_{s i} / E_{d} S i$ (eq.(6) over eq.(5)) to the approximate estimate of $\mathrm{E}_{\delta} \mathrm{Csl}_{\mathrm{l}} / \mathrm{E}_{\mathrm{d}} \mathrm{Si}$ (eq.(9) over eq.(8)). The initial spectrum must be known to perform integration in eq.(5), (6). Assume the initial X-ray spectrum of the following form.

$$
I_{0}(E)=A\left(\frac{1}{E}-\frac{1}{E_{0}}\right)
$$

The thickness of Si and CsI used in this analysis are $210 \mu \mathrm{m}$ and $461 \mu \mathrm{m}$, respectively. The thickness of $\mathrm{Al}$ is adjusted so that the average energy absorbed in $\mathrm{Al}$ as calculated below becomes equal to the energy determined by the experiment. The term $\mathrm{I}\left(\mathrm{E}, \mathrm{t}_{\mathrm{Al}}\right)^{*} \mathrm{E}$ and the terms in the integrals in eq.(5) and (6) are plotted in Fig.4-6. The discontinuity in the EsI curve is due to the $\mathrm{K}$ edge absorption of $\mathrm{Cs}$ and $\mathrm{I}$, which are around $34 \mathrm{keV}$. The centroid of the CsI curve is shifted to right compared to that of the Si curve, namely the average energy absolbed in CsI is higher than that in $\mathrm{Si}$. The areas under the CsI and $\mathrm{Si}$ curves give the energy deposited in each material and the ratio is 16.28 . 


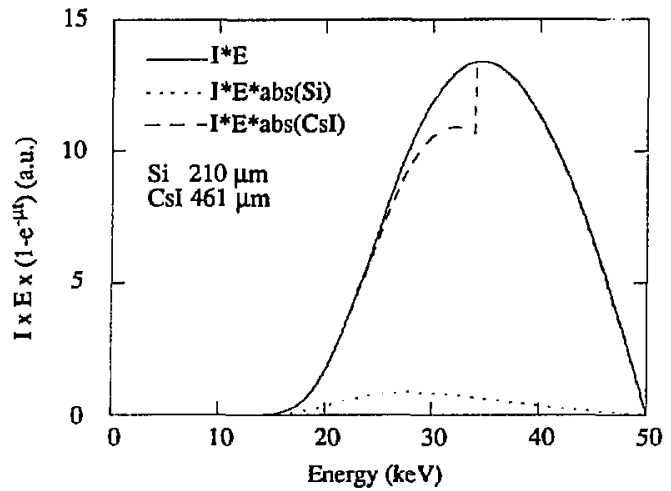

Fig.4-6 Absorption of X-ray spectrum by $\mathrm{CsI}$ and Si.

When we use the average energy model, the equivalent ratio is calculated as follows. The average energy is determined by the Al absorption curve. The following equation is used to calculate the average energy.

$$
\overline{\mathrm{E}}=\frac{\int_{0}^{\mathrm{F}_{0}} I\left(E, t_{A 1}\right) E\left[1-\exp \left(-\mu_{\mathrm{Al}}(E) t_{A 1}\right)\right] d E}{\int_{0}^{E_{0}} I\left(E, t_{A 1}\right)\left[1-\exp \left(-\mu_{A I}(E) t_{A l}\right)\right] d E}
$$

Its value for this example is adjusted to $31.1 \mathrm{keV}$. The average energies deposited in $\mathrm{Si}$ and CsI are calculated in a similar way and these are $29.0 \mathrm{keV}$ and $33.3 \mathrm{keV}$, respectively. Based on this average energy determined by $\mathrm{Al}$, the ratio of energy deposited in $\mathrm{CsI}$ and $\mathrm{Si}$ is calculated from eq.(8) and (9). This value is 13.77 and the correction factor becomes 1.18 . 


\section{4-4. Light yield optimization}

\section{4-4-1. Light yield of CsI(Na)}

The light yields from the $300 \mu \mathrm{m}$ thick $\mathrm{CsI}(\mathrm{Na})$ layers evaporated at $\mathrm{LBL}$ and from a single crystal CsI(T) made by Bicron (AI-409) are compared. AI-409 is a $5 \mathrm{~mm}$ thick 5 mm diameter cylinder-shaped crystal encapsulated by an Aluminum housing with $500 \mu \mathrm{m}$ thick window. The table below shows the signal size from the photodiode coupled to these scintillators and exposed to $\mathrm{X}$-ray pulses. The observed signal size measured in the photodiode is $S_{m}$. This is divided by $E_{d} C_{s I}$ to give the total electron yield per unit energy absorbed.

Table 4-5 Light yield of some evaporated CsI(Na)

\begin{tabular}{|c|c|c|c|}
\hline CsI type & $\begin{array}{c}\mathrm{E}_{\mathrm{d}} \mathrm{CI} \\
(\mathrm{MeV})\end{array}$ & $\begin{array}{c}\mathrm{S}_{\mathrm{m}} \\
\text { (electrons) }\end{array}$ & $\begin{array}{c}S_{\mathrm{m}} / \mathrm{E}_{\mathrm{d}} \mathrm{SI} \\
\text { (electrons } / \mathrm{MeV} \text { ) }\end{array}$ \\
\hline CsI(Na)\#120489 & 9.9 & $8.6 \times 10^{+4}$ & $8.7 \times 10^{+3}$ \\
\hline CsI(Na)\#121289 & 9.9 & $8.9 \times 10^{+4}$ & $9.0 \times 10^{+3}$ \\
\hline CsI(T) AI-409 & 7.1 & $1.5 \times 10^{+5}$ & $2.1 \times 10^{+4}$ \\
\hline
\end{tabular}

A handling problem exists for an evaporated CsI(Na) layer. It absorbs moisture upon contact with air and its light yield decreases with time. Scintillation stopped completely after it was left in air for a few days. Sealing it with an Al housing in an Ar-filled glove box after a shor exposure to air ( $<1 \mathrm{~min}$.) retarded the degradation but still its light yield decreased to a half value in a week or so. A mild baking in air $\left(200^{\circ} \mathrm{C}\right.$ for 2 hours) recovered light yield for unsealed layers but not for the sealed layers. Due to this hygroscopic nature of $\operatorname{CsI}(\mathrm{Na})$, optimization of the $\operatorname{CsI}(\mathrm{Na})$ evaporation process was not successful. Optimization of the CsI(Tl) evaporation process was carried out because $\mathrm{CsI}(\mathrm{Tl})$ is much less hygroscopic. 


\section{4-4-2. Light yield of CsI(Tl)}

The light yields of three crystalline CsI(Tl) samples supplied frum Bicron and a number of evaporated $\mathrm{Csl}(\mathrm{Tl})$ layers were evaluated with the $50 \mathrm{kV} \mathrm{X}$-ray pulse. A thin $\mathrm{Al}$ sheet covers the top surface of CsI(TI) layers so that it reflects back the scintillation light. The Iesults are summarized chronologically in Fig.4-7, showing the evolution of signal yield with time. More detailed data for some evaporated layers are tabulated in the Appendix at the end of chapter.

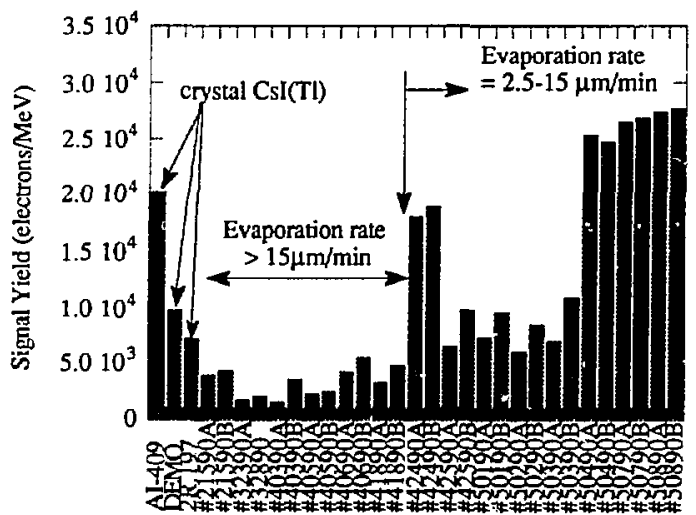

CsI(Tl) samples with Al reflector

Fig. 4-7 Signal size per unit energy absorbed in a number of CsI(TI) samples (their ID \#'s are also production dates in most cases).

The first three (AI-409, DEMO, 2R.197) are single crystal CsI(TI) supplied by Bicron. AI-409 is the one described above. $2 \mathrm{R} .197$ is a $5 \mathrm{~mm}$ thick $5 \mathrm{~cm}$ diameter disk of CsI(Tl) encapsulated by an aluminum housing with $500 \mu \mathrm{m}$ window. DEMO has the same dimensions with 2R.197 but its $5 \mathrm{~mm}$ CsI(T1) disk is cut by a wire-saw into $2 \mathrm{~mm} \times 2 \mathrm{~mm}$ columns and the gaps between columns are filled with reflector materials before encapsulation. The thickness of evaporated layers ranges from $100 \mu \mathrm{m}$ to $550 \mu \mathrm{m}$. 
As for the three single crystal CsI(T1) samples, it is possible that the lateral light spread inside the crystal resulted in the smaller signal size for DEMO and 2R.197. Among the evaporated CsI(T1) layers, the first twelve samples were evaporated at a rate faster than 15 $\mu \mathrm{m} / \mathrm{min}$. The last sixteen samples were evaporated at a well-controlled constant rate (about $2.5-15 \mu \mathrm{m} / \mathrm{min}$ ) and the source crystal was large enough so that there was some residual material left in the boat at the end of a run. Samples labeled as A were centered against the boat and two to three times thicker than the ones labeled as B, which were off-centered. The evaporation rate is based on the thicker "A" samples. The signal size for these layers is increased by as much as about $50 \%$ by putting a thin aluminanized mylar sheet as a reflector on top of the CsI(Tl) layers.

The light yield of $\operatorname{CsI}(\mathrm{TI})$ is sensitive to the evaporation conditions, especially the boat temperature which determines the evaporation rate. CsI(TI) layers $230-460 \mu \mathrm{m}$ thick were evaporated at different boat temperature, and hence different evaporation rates and signal yields were evaluated with the 50kV X-ray. The results are shown in Fig.4-8. A more detail data for some evaporated layers are tabulated in Appendix at the end of chapter.

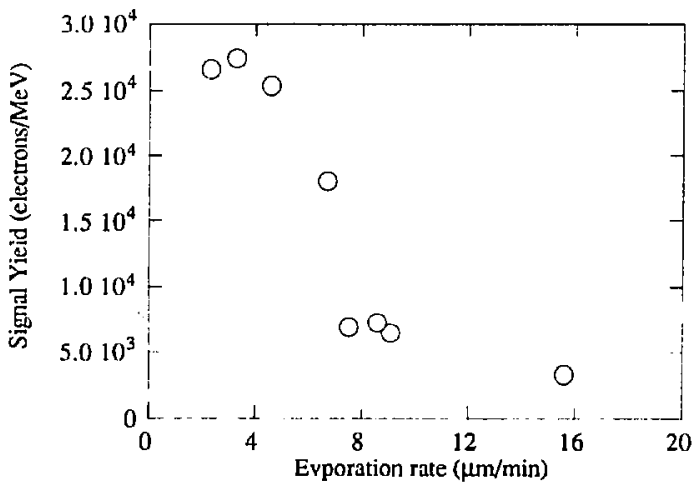


Fig. 4-8 Signal size per unit energy absorbed in CsI(Tl) evaporated at different constant rates. The rate is based on the samples labeled as $A$ which were centered against the boat.

In the temperature range around the melting point of $\operatorname{CsI}\left(620^{\circ} \mathrm{C}\right)$, the vapor pressure of TII is about four order of magnitude higher than that of CsI whereas Nal vapor pressure is almost the same as CsI [5]. This suggests that the poor scintillation quality of most of the early samples in Fig.4-7 was likely due to depleting $\mathrm{Tl}$ at the later part of a run. However, with optimum preparation, the light yield of evaporated CsI(TI) layers can be made close to a single crystal $\mathrm{CsI}(\mathrm{T})$ as demonstrated by some samples made at later time.

\section{4-4-3. TI concentration in an evaporated CsI(TI)}

The $\mathbf{T l}$ concentration is known to control the light yield in crystalline $\mathrm{CsI}(\mathrm{Tl})$ [11]. The $\mathrm{Tl}$ concentrations in our evaporated layers were evaluated by the X-ray Reflection Fluorescence analysis (XRF) [12]. The results are plotted in Fig.4-9 against their signal yields from the $\mathrm{X}$-ray measurements. The same behavior is seen for our evaporated layers and the crystalline CsI(TI) in ref.[11]. It is confismed that the layers evaporated at a slower rate contains more $\mathrm{Tl}$ than those made at a faster rate. The XRF measurement showed that the $\mathrm{CsI}(\mathrm{Tl})$ layers evaporated at slow rate contained $20-30 \%$ less $\mathrm{Tl}$ than their source CsI(TI) crystal. A more detail data for some evaporated layers are tabulated in Appendix at the end of chapter. 


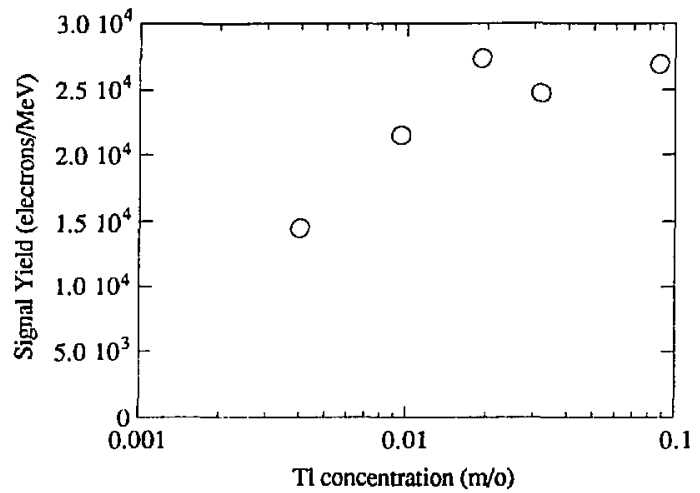

Fig.4-9 Signal size per unit energy absorbed in $\mathrm{CsI}(\mathrm{Tl})$ evaporated at different $\mathrm{Tl}$ concentrations as measured by XRF.

\section{4-5. Speed of CsI(TI) and $\mathrm{Gd}_{2} \mathrm{O}_{2} \mathrm{~S}$ screens}

The scintillation yields of our evaporated layers were characterized in the previous section. How fast scintillation decays is another important property of a scintillator. Although the decay constant of crystalline CsI has been well characterized, it was of our interest to confirm that our evaporated CsI(Tl) has a similar decay constant (about $1 \mu \mathrm{s}$ ) with its crystal counterpar.

There is another type of scintillator that has recently used in conjunction with a- $\mathrm{Si}: \mathrm{H}$ photodiodes. Hitachi has developed a compound of $\mathrm{Gd}_{2} \mathrm{O}_{2} \mathrm{~S}$ by introducing doping atoms such as $\mathrm{Pr}$, Ce and F and reported that its light yield is comparable to CsI(TI) and that its scintillation decays to $1 / 10$ of the initial level in $5 \mu \mathrm{s}$ [13]. Lanex $\left(\mathrm{Gd}_{2} \mathrm{O}_{2} \mathrm{~S}\right.$ :Tb) made by Kodak is an intensifying screen for X-ray films. Although $1-2 \mu \mathrm{m}$ thick a-Si:H photodiodes coupled to Lanex have been shown to give a IOO-330 times larger signal size for detecting $X$-rays used in medical imaging compared to the a-Si:H diode alone $[14,15]$, neither the absolute light yield nor the decay constant of Lanex were quoted and Lanex is 
known to have a long decay. How these two compounds of $\mathrm{Gd}_{2} \mathrm{O}_{2} \mathrm{~S}$ compared with our evaporated layer was interest to us.

The decay constant and light yield of a scintillator can be obtained by analyzing the charge-sensitive preamplifier output in the setup described in Ch.4-2 when the scintillator coupled to a photodiode is irradiated by a short $X$-ray pulse. The following experiment and analysis were conducted to compare these quantities of our evaporated CsI(TI) layers with those of $\mathrm{Gd}_{2} \mathrm{O}_{2} \mathrm{~S}$ screens.

\section{4-5-1. Theoretical analysis}

A scintillator may have multiple scintillation components each with its own decay constant, corresponding to a particular carrier transition between energy levels. It is of ten sufficient, however, to treat the scintillation yield as a single exponential decay with a characteristic time $\tau$, namely, $Y(t)=Y_{0} \exp (-t / \tau)$. We expect that the decay constants of $\mathrm{CsI}(\mathrm{Tl})$ and the Hitachi scintillator are comparable with the minimum setting of our $\mathrm{X}$-ray pulse $\left(T_{0}=1-2 \mu \mathrm{s}\right)$, whereas, the RC decay constant of our charge-sensitive preamplifier ( $R C=1 \mathrm{~ms}$ ) is comparable to the decay constant of Lanex. Since different assumptions will be made in the analysis, these are treated separately.

Case I. $\quad \tau \approx \mathrm{T}_{\mathrm{o}} \ll \mathrm{RC}(\mathrm{CsI}(\mathrm{Tl})$ and the Hitachi scintillator)

The RC discharge of the charge stored in the feedback capacitor of a charge-sensitive preamplifier can be neglected for $\tau \ll R C$. But the $X$-ray generation cannot be regarded as instantaneous.

The $X$-ray pulse of duration $\Delta t_{0}$ at $t=t_{0}\left(0 \leq t \leq T_{0}\right)$ yields scintillation light expressed as

$$
Y_{0} \exp \left(-\frac{t-h_{0}}{\tau}\right) \Delta t_{0}
$$

This light creates charge in the external circuit $\Delta Q\left(t, t_{0}\right)$ given by,

$$
\Delta Q\left(t, t_{0}\right)=A \int_{t_{0}}^{t} Y_{0} \exp \left(-\frac{t^{\prime}-t_{0}}{\tau}\right) d t^{\prime} \Delta t_{0}
$$


where, $\mathrm{A}$ is the factor involving the light collection and quantum efficiencies.

The total charge created by the $X$-ray pulse of duration $T_{0}$ is expressed as,

$$
\begin{array}{ll}
Q(t)=\int_{0}^{t} \Delta Q\left(t, t_{0}\right) d t_{0} & \text { for } 0 \leq t \leq T_{0} \\
Q(t)=\int_{0}^{T_{0}} \Delta Q\left(t, t_{0}\right) d t_{0} & \text { for } T_{0} \leq t
\end{array}
$$

These integrals are performed to give the following results.

$$
\begin{array}{ll}
Q(t)=Y_{0} \tau^{2}\left\{\frac{t}{\tau}-1+\exp \left(-\frac{t}{\tau}\right)\right\} A & \text { for } 0 \leq t \leq T_{0} \\
Q(t)=Y_{0} \tau^{2}\left[\frac{T_{0}}{\tau}-\exp \left(-\frac{t}{\tau}\right)\left(\exp \left(\frac{T_{0}}{\tau}\right)-1\right\}\right] A & \text { for } T_{0} \leq t
\end{array}
$$

The total charge observed at infinite time is given by,

$$
Q_{0}=Y_{0} \tau T_{0} A
$$

A dimensionless expression for the preamplifier output is obtained by dividing above expressions by $Q_{0}$.

Case Il. $\quad T_{0} \ll \tau \approx R C$ (Lanex)

In this case, the $\mathrm{X}$-ray generation is assumed as instantaneous but the $\mathrm{RC}$ decay of the charge stored in the feedback capacitor cannot be neglected. The preamplifier output is given by the scintillation decay function $Y(t)=Y_{0} \exp (-t / \tau)$ convolved by the response function of the charge-sensitive preamplifier $\exp (-t / R C)$, namely,

$$
Q(t)=\int_{0}^{t} Y_{0} \exp \left(-\frac{t_{0}}{\tau}\right) \exp \left(-\frac{t-t_{0}}{R C}\right) d t_{0}
$$

Therefore,

$$
Q(t)=Y_{0} \tau^{t}\left[\exp \left(-\frac{t}{R C}\right)-\exp \left(-\frac{t}{\tau}\right)\right]
$$

where,

$$
\frac{1}{\tau^{\prime}}=\frac{1}{\tau} \cdot \frac{1}{\mathrm{RC}}
$$


It should be noted that eq.(20) becomes a simple exponential form with a decay constant $\mathrm{RC}$ in the limit of $\tau \ll R C$. This is the case for the CsI(Tl) layer with $\tau=1 \mu$ s and RC approximately $1 \mathrm{msec}$.

\section{4-5-2. Measurement}

The same setup as shown in Fig.4-4 was used for this experiment except for that the charge-sensitive preamplifier (Tennelec TC170) output was directly fed into a digital storage oscilloscope (Tektronix 2430) and that digitized wave forms were analyzed by a personal computer. A crystalline Si radiation detector and the Hamamatsu photodiode (S1723-04) are placed in $\mathrm{CH} 1$ and $\mathrm{CH} 2$, respectively. A scintillator (either $\mathrm{Gd}_{2} \mathrm{O}_{2} \mathrm{~S}: \mathrm{PrCeF}$, Lanex or an evaporated CsI(TI) layer) was placed on top of S1723-04 and was exposed to the $1.4 \mu \mathrm{s} 50 \mathrm{kV}$ X-ray pulses as before (summarized in Table 4-2). The repetition rate of the $\mathrm{X}$-ray pulses were kept low to avoid pile up of output wave forms, which were stored and analyzed by a personi? computer.

First, the Hitachi scintillator $1.1 \mathrm{~mm}$ thick and our evaporated CsI(Tl) $\# 52490 \mathrm{~A} 461 \mu \mathrm{m}$ thick were compared and their waveforms are shown in Fig.4-10 together with the signal from the c-Si diode alone. Both scintillators absorbed all the X-ray energy. The linear portion of the c-Si signal extends about $1.2 \mu \mathrm{s}$, confirming the duration of a constant X-ray intensity. The asymptotic levels for these scintillators roughly agree and equivalent to $2.5 \times 10^{+4}$ electrons $/ \mathrm{MeV}$. The Hitachi scintillator is slightly slower than the CsI(Tl). The decay constants will be given in the next section. 


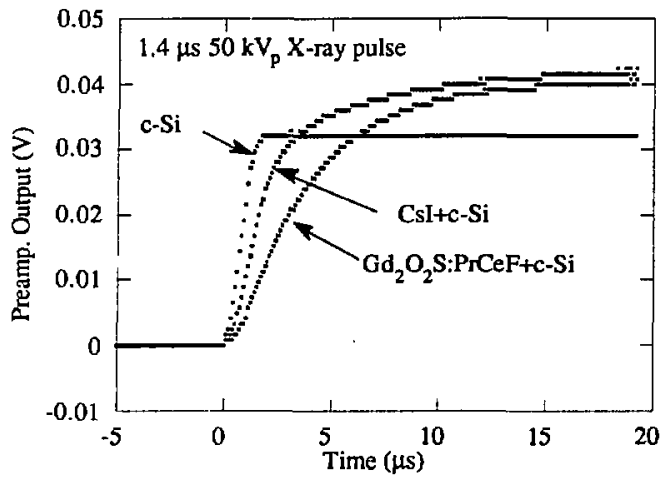

Fig.4-10 Preamplifier output for the CsI(Tl) layer and the Hitachi screen.

Second, Lanex and our CsI(TI) layer (\#50890A) were compared. The Lanex screen was about $300 \mu \mathrm{m}$ thick and mounted on a thin plastic The CsI(Tl) was $315 \mu \mathrm{m}$ thick and were e,vaporated on $1 \mathrm{~mm}$ thick glass. Unless a scintillator absorbs all the $\mathrm{X}$-ray energy, the signal contains a contribution from direct electron-hole pair creation by the genetrating $\mathrm{X}$-rays. This is the case for a $300 \mu \mathrm{m}$ thick Lanex screen and the direct signal contribution was measured as previously by placing a thin opaque sheet betweer a scintillator and S1723-04. The resultant wave forms were subtracted from the uave forms observed without the opaque sheet. The average energy of the $\mathrm{X}$-ray is $30 \mathrm{kcV}$ and the incoming energy contained in a pulse is equivalent to $10 \mathrm{MeV}$. Under these conditions, the direct contribution was about $20 \%$ of the total charge collected by the photodiode in the case of the Lanex screen and $10 \%$ for the CsI(TI) layer. The wave forms after subtraction of the direct contributions are shown in Fig.4-11. The decay constant RC is about $1 \mathrm{~ms}$ and this can be determined more accurately from the CsI(TI) signal as shown below. The Lanex signal shows a peak and then decays more slowly than the CsI signal. This is 
because the scintillation characteristic time is comparable with the decay constant of the preamplifier.

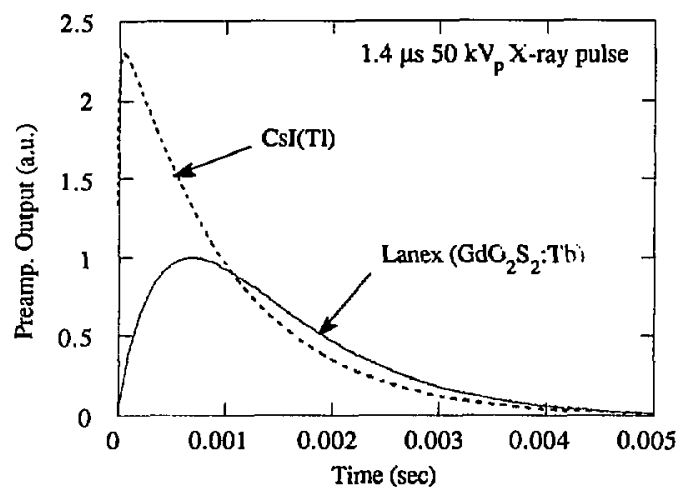

Fig.4-11 Preamplifier output for the CsI(Tl) layer and Lanex. Both curves were normalized against the peak value of the Lanex data.

\section{4-5-3. Analysis}

First, the wave forms in Fig.4-10 were normalized against their asymptotic values to give dimensionless output forms and their initial portions during the $X$-ray pulse $(0 \leq t \leq$ $T_{0}$ ) were fitted with the theoretical expression. The decay constant $\tau$ is the only fitting parameter used and results with three different $\tau$ are plotted in the Fig.4-12 for the two scintillators. The best fit is given by $\tau=1.1 \mu$ s for the CsI(TI) and $\tau=3.5 \mu$ s for the Hitachi scintillator, respectively. These values agree well with published data. 


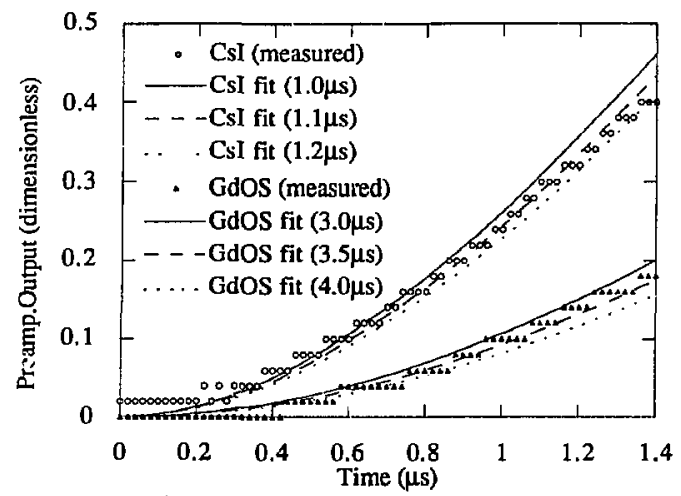

Fig.4-12 Dimensionless preamplifier output fitted with the theoretical expression.

Second, the Lanex data were analyzed. Fitting the exponential tail of the CsI(TI) signal gives the RC decay constant of $1.06 \mathrm{~ms}$. The Lanex signal can be fitted by eq.(20) using

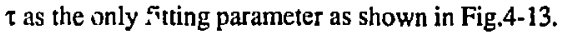

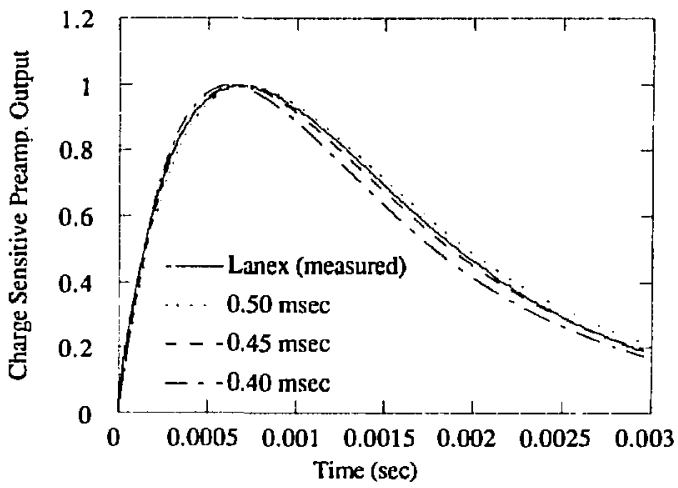

Fig.4-13 Fitting the Lanex signal by the analytical expression for the preamplifier output. 
Since the wave form is well described by eq.(20), the peak value ( $\left.t_{\max }, Q_{\max }\right)$ can be used to calculate $\tau$ for Lanex. The expression for these are given by,

$$
\begin{aligned}
& \tau=\mathrm{RC} \exp \left(-\frac{\mathrm{t}_{\max }}{\tau^{\prime}}\right) \\
& \mathrm{Q}_{\max }=\mathrm{Y}_{\mathrm{a}} \tau\left(\frac{\tau}{\mathrm{RC}}\right) \frac{\tau^{\prime}}{\mathrm{RC}}
\end{aligned}
$$

From the peak value $t_{\max }$ of $0.700 \mathrm{msec}$ for Lanex, the decay constant of $0.48 \mathrm{msec}$ is obtained by solving eq.(23) graphically.

The total light yield is given by

$$
\int_{0}^{\infty} Y_{0} \exp \left(-\frac{t}{\tau}\right) d t=Y_{0} \tau
$$

The ratio of the total light yield of CsI(TL) to that of Lanex is calculated by eq.(23) and eq. (24) from the peak values $\mathrm{Q}_{\max }$ of both wave forms. This ratio is 1.19.

In conclusion, it has been shown that the evaporated $\mathrm{CsI}(\mathrm{Tl})$ has a total light yield equivalent to the Hitachi scintillator and Lanex and that its decay constant is $1.1 \mu \mathrm{s}$ whereas those of Hitachi scintillator and Lanex are $3.5 \mu \mathrm{s}$ and $480 \mathrm{us}$, respectively.

\section{4-6. Spatial resolution measurement}

The columnar structure in evaporated CsI(Na) layers [6] was confirmed by a Scanning Electron Micrograph (SEM) of a cracked surface of the layer. Light spread inside a $\mathrm{CsI}(\mathrm{Na})$ layer was characterized qualitatively by taking a photograph of a reflected light pattern when the top surface of the $\operatorname{CsI}(\mathrm{Na})$ was irradiated by a narrow light beam [6]. A simiiar structure and light spreas were expected from our evaporated CsI(TI) and the following two experiments were conducted to confirm these points.

First, the columnar structure of evaporated CsI(TI) layers and the effect of the substrate temperature on the structure were checked by a SEM. A small piece of the evaporated layers were cut with a razor blade and the cracked surfaces were observed with a SEM. The two SEM photographs shown in Fig.4-14 clearly show the columnar structure. The 
substrate temperature during the evaporation was $100^{\circ} \mathrm{C}$ for Fig.4-14(a) and $200^{\circ} \mathrm{C}$ for Fig.4-14(b). The evaporation parameters for the $100^{\circ} \mathrm{C}$ layer were optimized for maximum light yield. Only the substrate temperature was raised to $200^{\circ} \mathrm{C}$ for the layer shown in Fig.4-14(b). The columnar diameter of the $100^{\circ} \mathrm{C}$ layer is about $10 \mu \mathrm{m}$ whereas that of the $200^{\circ} \mathrm{C}$ layer is about $15-20 \mu \mathrm{m}$. The light yield of the $200^{\circ} \mathrm{C}$ layer was almost the same as the $100^{\circ} \mathrm{C}$ layer. 
Fig.4-14 SEM photograph of cracked surfaces of CsI(TI) layers evaporated at substrate temperature of $100^{\circ} \mathrm{C}(\mathrm{a})$ and $200^{\circ} \mathrm{C}(\mathrm{b})$.
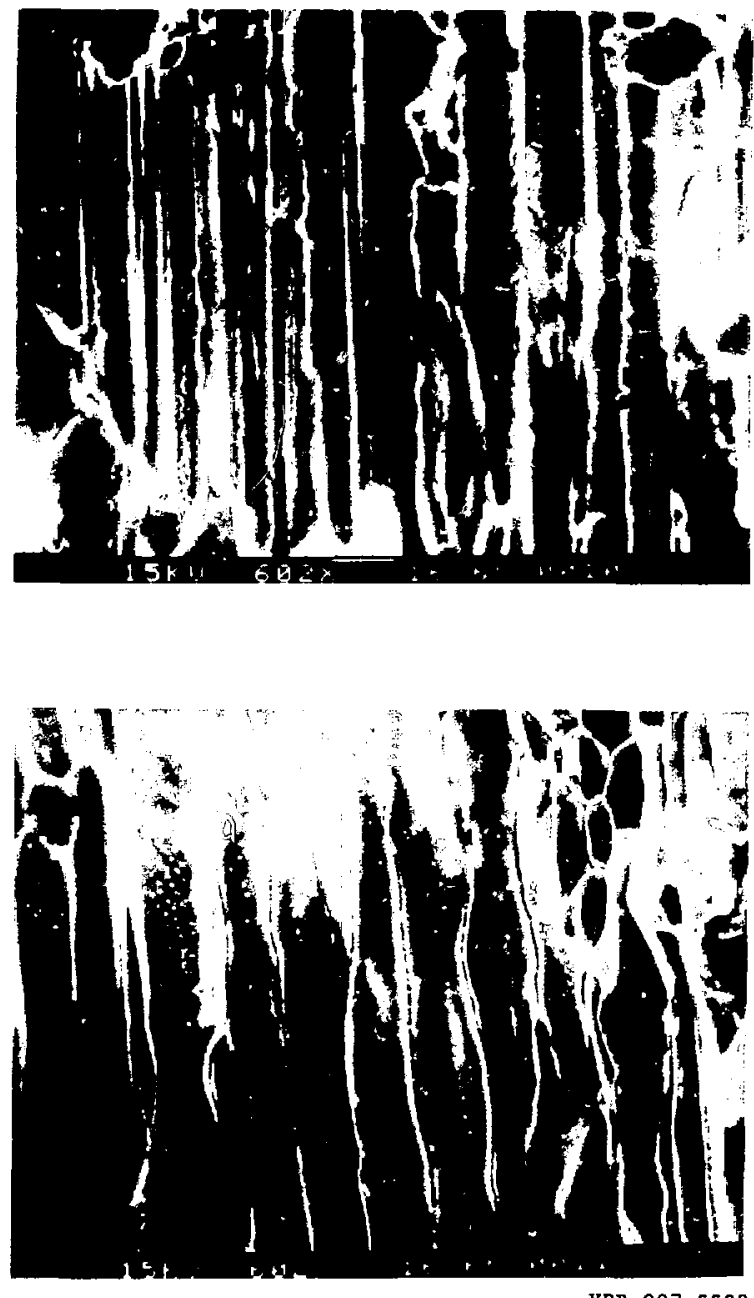

$\mathrm{XBB} \quad 907-5502$ 
Second, light spread inside an evaporated CsI(TI) layer as well as the Hitachi scintillator $\left(\mathrm{Gd}_{2} \mathrm{O}_{2} \mathrm{~S}: \mathrm{PrCeF}\right)$ and Lanex screen $\left(\mathrm{Gd}_{2} \mathrm{O}_{2} \mathrm{~S}: \mathrm{Tb}\right)$ used before were characterized by the setup shown in Fig.4-15. The slit was placed as close as possible to the surface of the scintillator and the distance between the slit and the scintillator surface was less than $1 \mathrm{~mm}$ in all the scintillators tested.

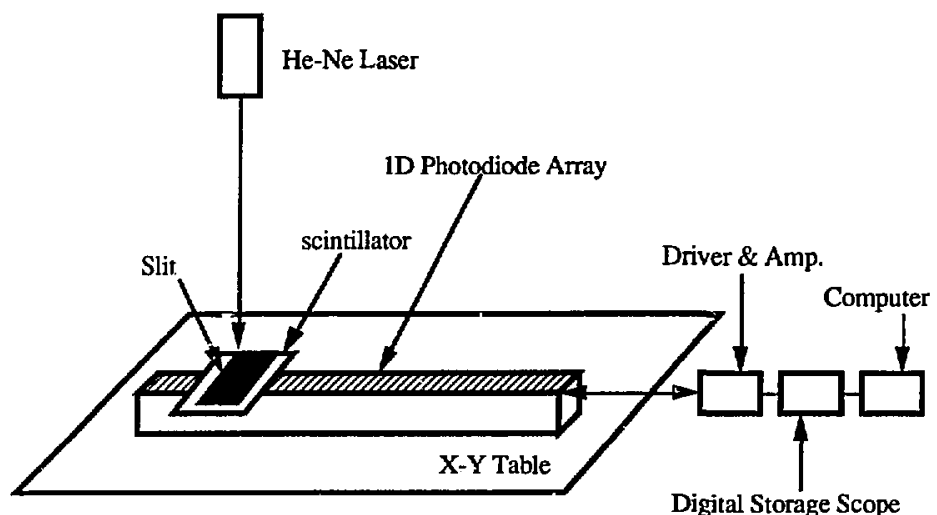

Fig.4-15 Experimental setup for the light spread measurement.

A facsimile head module made by SEIKO-EPSON (linear a-Si:H photodiode array with a TFT attached to each photodiode) had 400 dpi resolution [16]. The external circuit was the standard readout electronics designed for the facsimile application and it gave a sequence of voltage pulses, each pulse height corresponding to a charge produced by each photodiode element. This pulse sequence was digitized and stored by the Tektronix 2430 scope and then transfered to a computer for analysis.

First, the photodiode array was exposed to a parallel light beam from a He-Ne Laser through a narrow slit without a scintillator between the slit and the array. The slit opening was set to $50 \mu \mathrm{m}$ so that only one pixel of the linear photodiode array gave a signal. The position of the array was adjusted such that the signal becomes maximum. Second, a 
scintillator (either a $425 \mu \mathrm{m}, 894 \mu \mathrm{m}$ evaporated $\mathrm{CsI}(\mathrm{TI})$ layer or onte of the two $\mathrm{Gd}_{2} \mathrm{O}_{2} \mathrm{~S}$ compounds used before) was placed on the photodiode array and the Laser beam was used to expose the scintillator and the array. The intensity of the hean was adjusted by an optical attenuator sheet so that the signal was not saturated. An example of the output pulse sequence is shown in Fig.4-16.

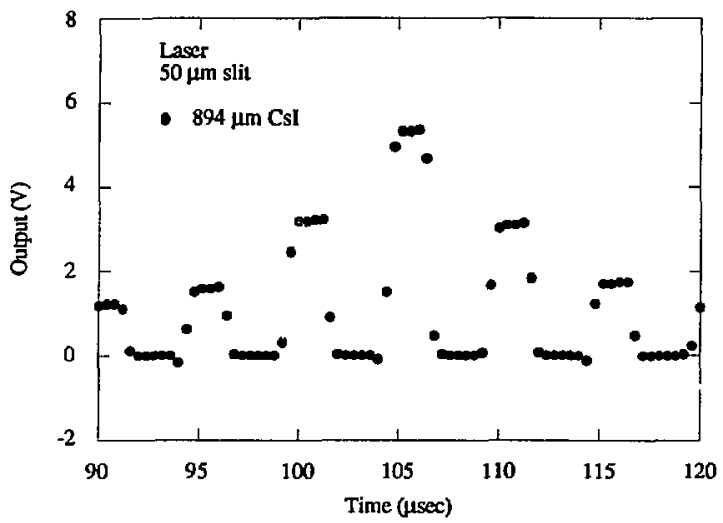

Fig.4-16 Digitized pulse sequence obtained by the setup shown in Fig.4-15 with a $894 \mu \mathrm{m}$ thick CsI(Tl) layer.

The flat portions of the pulse sequence in Fig.4-16 corresponds to the time when a thin film transistor (TFT) attached to every photodiode element is turned on or off. Notice that the switching transient exists as can be seen as a small distortion from an ideal rectangular pulse. For the Laser light, this distorion is negligible compared to the signal ctaage. Therefore, a line spread function can be obtained by sampling one representative data point from each flat portion of the pulse sequence corresponding to the time when the TFT is turned on. The time was converted into position by using the known pitch of the photodiode array. A normalized line spread function was obtained this way for each scintillator and is shown in Fig.4-17 and Fig.4-18. 


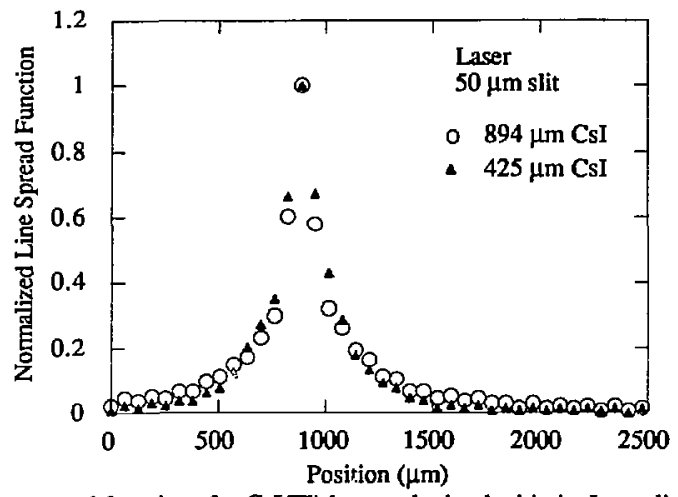

Fig.4-17 Line spread functions for CsI(T1) layers obtained with the Laser light beam.

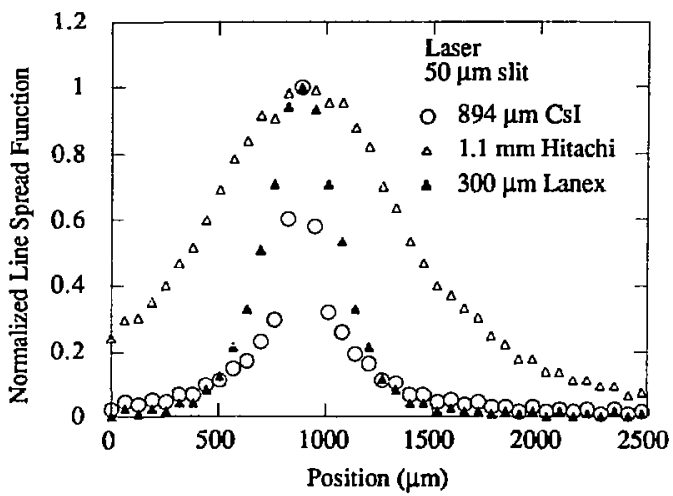

Fig.4-18 Line spread functions for the $\mathrm{CsI}(\mathrm{Tl})$ and the two $\mathrm{Gd}_{2} \mathrm{O}_{2} \mathrm{~S}$ compounds obtained with the Laser light beam. The spatial resolution of the $894 \mu \mathrm{m}$ thick evaporated CsI(T1) layer is about $200 \mu \mathrm{m}$ FWHM, whereas those of the $\mathrm{Gd}_{2} \mathrm{O}_{2} \mathrm{~S}$ compounds are $400 \mu \mathrm{m}$ FWHM for the $300 \mu \mathrm{m}$ thick Lanex screen and $1 \mathrm{~mm}$ FWHM for the $1.1 \mathrm{~mm}$ thick Hitachi scintillator. 
Spatial resolution is often expressed in terms of a full width at half maximum (FWHM) of a line spread function. The thicknesses and the approximate resolutions of all the scintillators tested here are summarized in Table 4-6.

Table 4-6 Sparial resolutioon measured by Laser light

\begin{tabular}{|c|c|c|}
\hline Scintillator & Thickness & spatial resolution \\
\hline evaporated Csl(TI) & $425 \mu \mathrm{m}$ & $200 \mu \mathrm{m}$ FWHM \\
\hline evaporated CSI(TI) & $894 \mu \mathrm{m}$ & $200 \mu \mathrm{m}$ FWHM \\
\hline Hitachi (Gd $\left.\mathrm{Gd}_{2} \mathrm{~S}: \mathrm{PrCeF}\right)$ & $1.1 \mathrm{~mm}$ & $1 \mathrm{~mm}$ FWHM \\
\hline Lanex $\left(\mathrm{Gd}_{2} \mathrm{O}_{2} \mathrm{~S}: \mathrm{Tb}\right)$ & $300 \mu \mathrm{m}$ & $300 \mu \mathrm{m}$ FWHM \\
\hline
\end{tabular}

This comparison of scintillators is not a clean quantitative one because the thickness of each scintillator is not the same, but it is clearly indicated that the light spread inside the evaporated (SI)T1) layer is suppressed by its columnar structure.

A similar experiment was conducted with an X-ray beam instead of the Laser. The 425 $\mu \mathrm{m}$ thick CsI(TT) layer coupled to the linear a-Si:H photodiode array was exposed to $50 \mathrm{kV}$ $X$-ray $(\mathrm{DC})$ through a $75 \mu \mathrm{m}$ tungsten slit. Now the switching transient is larger than the photodiode signal size and the output pulse sequence looks very different as shown in Fig.4-19. The open circles show the pulse sequence when the X-ray beam was on and the solid circles show the switching transient only when the $\mathrm{X}$-ray beam was off. Notice that the vertical scale of this figure is $1 / 1000$ of Fig.4-16. Apparently there are two alternating patterns of transient in Fig.4-19. This is presumably due to the subtle difference in the two alternating readout lines which are implemented to increase the readout time [16]. Since these switching transient exist systematically independent of whether the $\mathrm{X}$-ray beam is on or off, it can be subtracted from the observed $\mathrm{X}$-ray signal to give a clearer picture of a line spread function. A line spread function shown in Fig.4-20 was obtained by averaging the difference between the two pulse sequences during the TFT ON periods and by converting time into position. 


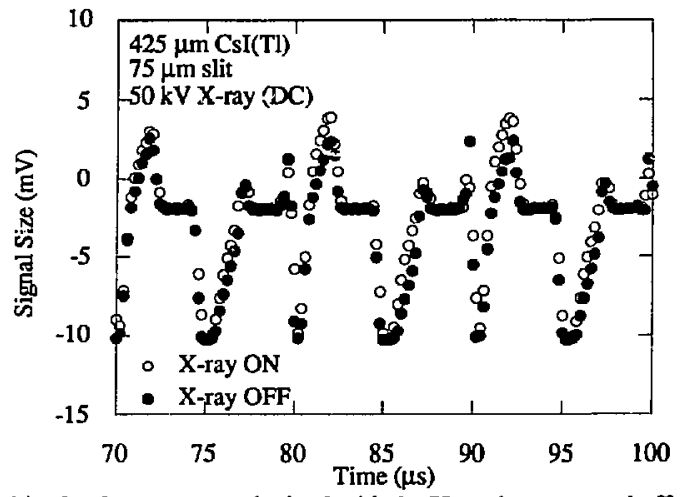

Fig.4-19 Digitized pulse sequence obtained with the $\mathrm{X}$-ray beam on and off.

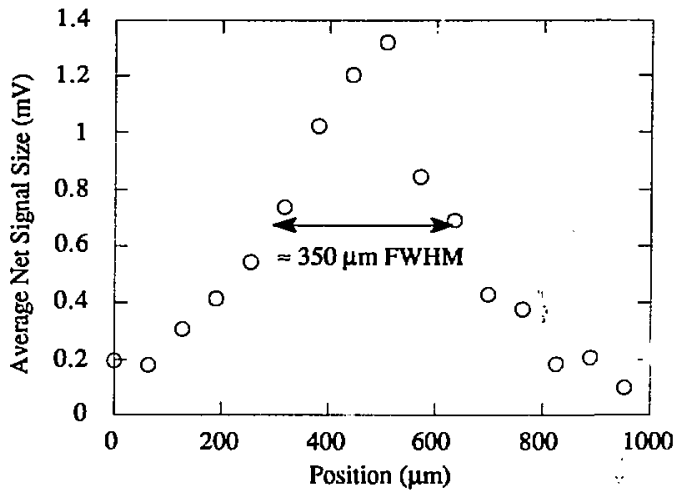

Fig.4-20 Line spread functions for the CSI(TI) obtained by average difference analysi of Fig.4-19.

A discrepancy between the Laser measurements and the $\mathrm{X}$-ray measurement comes from the fact that the scintillation light is emitted isotropically. Total reflection orsurs on the columnar surface if the incident angle of the light is larger than the critical angle. This is 
calculated to be $34^{\circ}$ for the CsI and air boundary [6]. Some of the scintillation light is trapped inside the scintillator by internal reflection. Most of the lighi (83\%) [6] come out from the CsI layer by successive reflection but the direction of this light at the end of the columns can make an angle as much as $34^{\circ}$ with respect to the column direction of the CsI layer. On the other hand, the Laser light is inciderit on the scintillator normally and a large angle scat tering on the columnar surface is unlikely, leading to a narrower light spread.

\section{4-7. Radiation damage to an evaporated CsI(T)}

A relatively thin evaporated CsI(Tl) (\#50890B $125 \mu \mathrm{m}$ thick) was exposed to $\gamma$ rays from a strong Co-60 source at the irradiation facility at LBL. The nominal activity of the Co-60 source is $6000 \mathrm{Ci}$. The $\mathrm{CsI}(\mathrm{Tl})$ sample was held at $10-30 \mathrm{~cm}$ away from the source and left for various time periods required for each dose. The signal size was measured with and without a reflector after each exposure and plotted in Fig.4-21. Also shown in the figure are the data for $1 \mathrm{~cm}$ thick crystal CsI(T1) taken from Ref.[17]. Each signal size is normalized against its zero dose level.

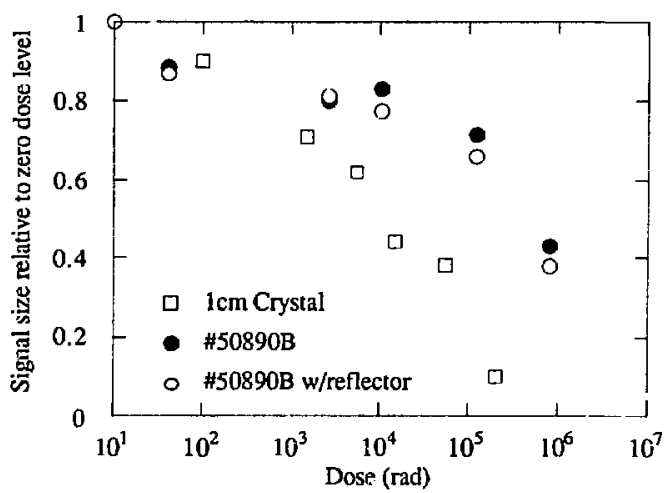

Fig.4-21 Light yield decrease due to radiation damage for a thin Csl(TI) iayer. 
The dose required for $60 \%$ signal loss is about $10^{+6} \mathrm{rad}$ for the evaporated layer, which is about 50 times more than the dose required for the $1 \mathrm{~cm}$ crystal sample. The signal loss is systematically larger when the light collection is improved by the reflector. This suggests that the radiation damage changed transmission properties of the scintillator, in agreement with a more detailed study of the crystal CsI(T1) [17].

\section{4-8. $X$-ray and $\beta$ particle detection with CsI coupled to a photodiode}

\section{4-8-1. X-ray detection}

The direct and indirect detection of X-ray pulses was compared using a $10 \mu \mathrm{m}$ a-Si:H pi-n diode and a $233 \mu \mathrm{m}$ thick evaporated CSI(Tl) layer. The structure of the diode was 0.8 $\mathrm{mm}$ glass $/ \mathrm{SnO}_{2} / 30 \mathrm{~nm}-\mathrm{p}+/ 10 \mu \mathrm{m}-\mathrm{i} / 30 \mathrm{~nm}-\mathrm{n}+/ \mathrm{Cr}$ and its top $\mathrm{Cr}$ contact was $3 \times 3$ $\mathrm{mm}^{2}$ square. The open circles in Fig.4-22 show the pulse height spectrum for direct $X$ ray detection without a scintillator when this a-Si:H p-i-n diode was reverse-biased at 100 $\mathrm{V}$ and exposed to the collimated $50 \mathrm{kV} \mathrm{X}$-ray beam directly. The average energy of $\mathrm{X}$-ray photons was $30 \mathrm{keV}$. The total energy contained in a single pulse was calibrated to be 22 $\mathrm{MeV}$. Next the glass side of this diode was coupled to the glass substrate of a $233 \mu \mathrm{m}$ thick CsI(Tl) (\#42490). The pulse height spectrum of the signal for the same X-ray 
exposure to the scintillator is shown by solid circles in Fig.4-22.

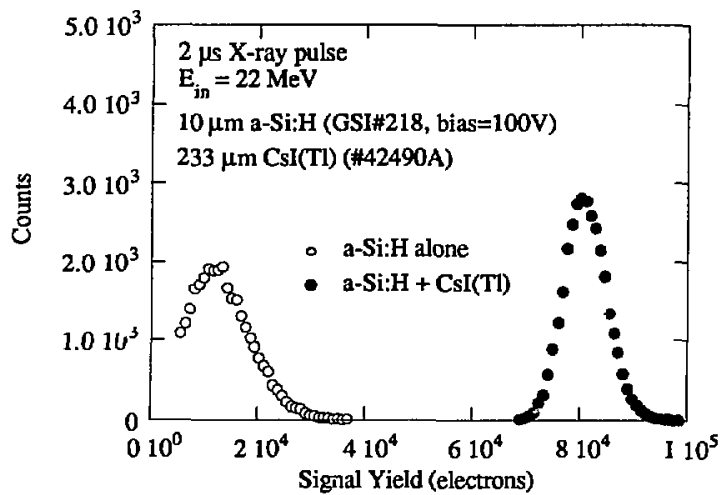

Fig.4-22 Detection of X-ray by a $10 \mu \mathrm{m}$ thick a-Si:H diode with and without a $233 \mu \mathrm{m}$ thick CsI(TI).

The direct signal size of about $1.2 \times 10^{+4}$ electrons is consistent with what we expect from $W$ (the average energy to create an electron-hole pair) of $5 \mathrm{eV}$. As for the scintillation signal size of about 3600 electrons $/ \mathrm{MeV}$, it is about $20 \%$ of the signal yield of the same CsI(T1) layer coupled to the Hamamatsu photodiode. This loss may be due simply to inadequate light collection by the a-Si:H diode. Despite this loss, signal enhancement of a factor of 8 over direct detection is achieved by using this scintillator.

\section{4-8-2. $\beta$ particle detection}

An evaporated CsI(Tl) layer $315 \mu \mathrm{m}, 461 \mu \mathrm{m}$ or $894 \mu \mathrm{m}$ thick was coupled to the Hamamatsu photodiode S1723-04. The $894 \mu \mathrm{m}$ layer was peeled off from the substrate and was coupled to the photodiode directly. Other layers were coupled through their roughened glass substrates. This photodiode was used, due to its small capacitance (50 $\mathrm{pF}$ ), to reduce the noise. A thin Al reflector was put on the scintillator for better light 
collection. This assembly was exposed to $\beta$ particles from a $\mathrm{Bi}-207$ source. The output of the photodiode was amplified by the charge-sensitive preamplifier (Tennelec TC170) and shaped by a quasi-Gaussian shaping amplifier ( $6.4 \mu \mathrm{s}$ peaking time). The resultant pulse height spectrum is shown in Fig.4-23. The solid and open circles and solid triangles in Fig.4-22 show the spectra obtained with $315 \mu \mathrm{m}, 461 \mu \mathrm{m}$ and $894 \mu \mathrm{m} \operatorname{CsI}(\mathrm{Tl})$ layer respectively. The open triangles show the spectrum when the source was out.

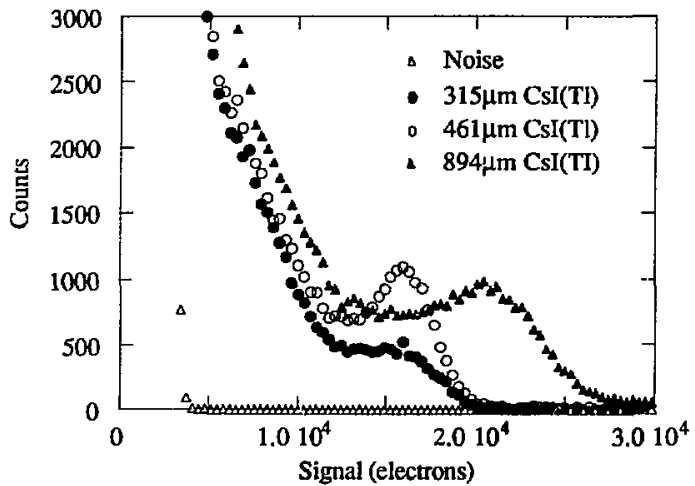

Fig.4-23 Detection of $\beta$ particies from a Bi-207 source by an evaporated CsI(TI) layer coupled to a photodiode.

A significant amount of low energy counts in Fig.4-23 for all of the three scintillators suggests that the a large number of electrons escape from these layers. When the layer thickness is comparable to the electron range, some electrons deposit their full energy, creating a peak in the pulse height spectrum [18]. The pulse height spectrum shown by the open circles in Fig.4-24 was obtained by tilting the $461 \mu \mathrm{m}$ CsI(Tl) coupled with S1723-04 by $60^{\circ}$ against the $\beta$ particle incident direction, thus by increasing the effective thickeness of CsI(Tl) by a factor of two. The fact that the peak appears at the saine energy confirms 
that this peak is the full energy peak. The $1 \mathrm{MeV}$ monoenergetic $\beta$ particles from the $\mathrm{Bi}$ 207 source create a peak only with the thicker CsI(T1) layers The $894 \mu \mathrm{m}$ layer gave a larger signal yield than the other two layers probably because of better coupling to the photodiode.

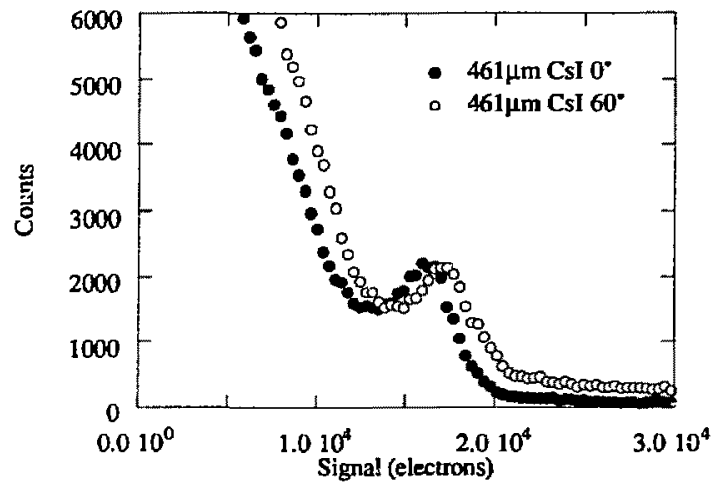

Fig.4-24 Detection of $\beta$ particles from a Bi-207 source by an evaporated CsI(T1) layer coupled to a photodiode for normal incidence and $60^{\circ}$ incidence.

Finally, the Hitachi scintillator ( $1.1 \mathrm{~mm}$ thick $\mathrm{GdO}_{2} \mathrm{~S}_{2}: \operatorname{PrCeF}$ ) was compared with the $461 \mu \mathrm{m} \mathrm{CsI(Tl)}$ for detecting $\beta$ particles from the Bi207 source. Almost the same pulse height spectra were obtained from the Hitachi scintillator as from the $461 \mu \mathrm{m}$ thick CsI(T1) layer shown in Fig.4-25. The previous analysis on the X-ray measurements showed that this Hitachi scintillator has almost the same signal yield with a slightly longer decay constant with CsI(Tl). The result of similar spectra for the two scintillators in Fig.4-25 is consistent with the $\mathrm{X}$-ray analysis on the signal yields and also with the discussion about the nature of the peak. 


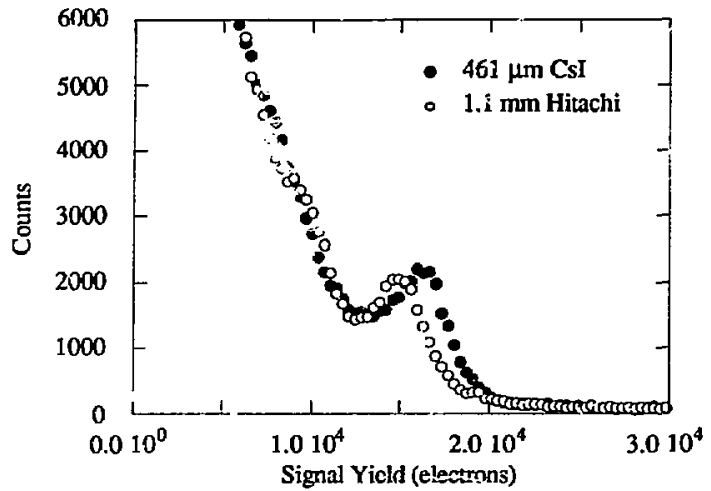

Fig.4-25 Detection of $\beta$ particles from a $\mathrm{Bi}-207$ source by an evaporated $\mathrm{CsI}(\mathrm{Tl})$ layer and the Hitachi scintillator coupled to a photodiode.

Based on detection of single $1 \mathrm{MeV} \beta$ parcicles, we can extraporate to project that high energy minimum ionizing particles will produce $\approx 10,000 \mathrm{e}-\mathrm{h}$ pairs for a $500 \mu \mathrm{m}$ thick Cr i(Tl) layer coupled to a $70 \%$ quan'um efficiency photodiode since they deposit about 500 $\mathrm{keV}$ in a $500 \mu \mathrm{m}$ thick CsI(T1) layer. The signal to noise ratio would be larger than 10 if noise can be reduced to less than 1,000 electrons. Noise is expected to be reduced by making a smaller aren device since the dominant component of noise is the delta noise, which is proportional to the detector capacitance [ref.4 of Chapter 3]. The individual element of an a-Si:H photodioce array would nave much smaller areas and therefore lower capacitance.

\section{4-9. Monolithic $\mathrm{X}$-ray sensor}

The scintillator materials were coupled to a photodiode through a glass or a transparent plastic plate in all the cases discussed so far. The Hamamatsu photodiode was extensively used beca'ise of the calibrated quantum efficiency and the low noise characteristics and it 
permited independent testing of phosphors. In combining the CsI with an a-Si:H photodiode, it is advantageous to have the CsI evaporated directly on an a-Si:H photodiode for (a) a better optical coupling between the two [19] and (b) no lateral spread of scintillation light. The structure of the monolithic $X$-ray sensor is schematically shown in Fig.4-26.

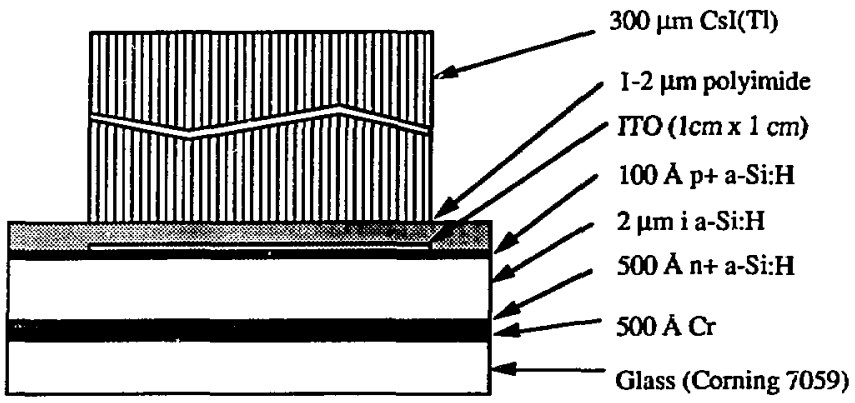

Fig.4-26 Monolithic X-ray sensor consisting of CsI(Tl) and a-Si:H photodiode.

The a-Si:H diode shown in Fig.4-26 was fabricated by the GD method. The top electronically conductive contact was made by sputtering about $1000 \AA$ thick of indium tin oxide (ITO) on the top surface. This forms an optically transparent and electronically conductive layer [20]. We found that the CsI evaporated directly on the ITO coated a-Si:H did not adhere well. Some coating materials such as $\mathrm{MgO}, \mathrm{SiO}_{2}, \mathrm{SiO}$ were evaporated on the ITO in the hope of improving the CsI adhesion but these attempts were unsuccessful. Finally, we tried a high temperature plaslic, polyimide. Polyimide layer has been used as a passivation layer for microelectronics applications. We found that it did improve adhesio of the CsI to thi ITO. The polyimide PI-2555 (DuPont) was spin-coated (at $6000 \mathrm{Ipm}$ for $30 \mathrm{sec}$ ) on the ITO layer and it was baked at $200^{\circ} \mathrm{C}$ for $30 \mathrm{~min}$. Although the polyimide layer looked slightly yellow, the absorption of the CsI(TI) scintillation was almost negligible. This was checked by comparing the signal yields when a polyimide layer 
coated glass was inserted between the scintillator and the photodiode and when a similar glass without polyimide coating was inserted. Finally, CsI(TI) $317 \mu \mathrm{m}$ thick was evaporated with the optimized parameters.

This monolithic device was exposed to the $50 \mathrm{kV}$ X-ray pulses. The pulse width was adjusted so that the energy contained in a single pulse was $10 \mathrm{MeV}$ or $20 \mathrm{MeV}$. The pulse height spectra were obtained under these conditions and are shown in Fig.4-27. The threshold was set at the signal level of $1.0 \times 10^{+5}$ electrons due to the large noise $\left(\approx 5 \times 10^{+4}\right.$ electrons FWHM) arising from the large capacitance of the a-Si:H diode ( $5000 \mathrm{pF})$. The signal yield of $1.2 \times 10^{+4}$ electrons/ $\mathrm{MeV}$ was obtained. This was about one half of the signal yield obtained by the other CsI(T1) layer evaporated at the same time and coupled to the Hamamatsu photodiode. The loss can be explained by inferior quantum efficiency of this particular a-Si:H diode because of light absorption in the $\mathrm{p}^{+} \mathrm{a}-\mathrm{Si}: \mathrm{H}$ layer. Based on the results by Weisfield and Tsai [21], making the $\mathrm{p}^{+}$layer with a-SiC:H rather than plain a-Si:H will reduce the p-layer absorption of the scintillation. light to less than $5 \%$.

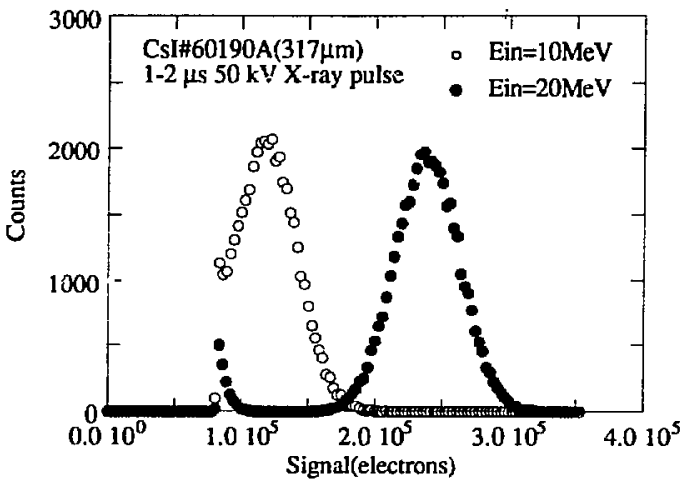

Fig.4-27 M:onolithic $X$-ray sensor consisting of $\mathrm{CsI}(\mathrm{TI})$ and a-Si: ${ }^{-} i$ photodiode. 


\section{4-10. Conclusion}

CsI(TI) layers $100-900 \mu \mathrm{m}$ thick were evaporated from crystal CsI(M) chips on glass substrates as well as on a-Si:H layers. They were found to give light yields and speed comparable to crystal CsI(T1). Concentration of $\mathrm{Tl}$ atoms in the evaporated layers was measured and correlated with the light yield. The light spread inside the scintillator was suppressed by the columnar structure of the evaporated CsI(TI) layers. A prototype monolithic X-ray sensor consisting of CsI(T1) and a-Si:H photodiode was fabricated. Further improvement in signal amplitude for this device is possible by optimizing the photodicde performance as well as the nptical collection efficiency. Full implementation for most applications will require reducing the area of individual diode elements for low noise operation. 


\section{Appendix Summary of various evaporation runs}

The table below summarizes the CsI(T) fabrication and measurement data. The first column (CsI\#) shows identification numbers for our evaporated layers. The first three are crystal CsI(TI) made by Bicron. The rest is our evaporated CsI(TI) under various conditions. The $\mathrm{ID}$ \# is also the date of evaporation run. Samples labeled as " $\mathrm{A}$ " were centered over the boat and those labeld "B" were off-centered except for the \#21590" samples which were both off-centered. The thicknesses shown in the second column were calculated from the measured weight increase of the glass substrates, assuming the sane density of $\mathrm{CsI}(\mathrm{Tl})\left(4.51 \mathrm{~g} / \mathrm{cm}^{3}\right)$. The physical thickness measured by micrometer was 10 $20 \%$ larger that these calculated values, indicating the density of evaporated layers is $10-20$ \% less than crystal CsI(TI). The signal yield was measured using the $50 \mathrm{kV} X$-ray pulse and is expressed in terms of electrons per unit energy absorbed in the scintillators. A thin Al reflector was placed on top of the scintillator to measure the yields shown in the fourth column. The evaporation rate was obtained by dividing the thickness of the " $\mathrm{A}$ " sample by the evaporation time. The Tl concentration was obtained by the X-ray Reflection Fluurescence (XRF) analysis.

\section{Table Summary of various evaporation runs}

\begin{tabular}{|c|c|c|c|c|c|}
\hline CSI\# & $\begin{array}{c}\text { Thickness } \\
(\mu \mathrm{m})\end{array}$ & $\begin{array}{c}\text { yield w/o AI } \\
(\mathrm{e} / \mathrm{MeV})\end{array}$ & $\begin{array}{c}\text { yield w/Al } \\
(\mathrm{e} / \mathrm{MeV})\end{array}$ & $\begin{array}{c}\text { Evp.rate } \\
(\mu \mathrm{m} / \mathrm{min})\end{array}$ & T1 (m/o) \\
\hline AI-409 & 5000 & & 20246 & & \\
\hline DEMO & 5000 & & 9796 & & \\
\hline 2R.197 & 5000 & & 7184 & & \\
\hline$\# 21590 \mathrm{~A}$ & 307 & & 3953 & & \\
\hline$\# 21590 \mathrm{~B}$ & 307 & & 4282 & & \\
\hline$\# 32390 \mathrm{~A}$ & 165 & & 1760 & & \\
\hline$\# 32890$ & 532 & & 2027 & & \\
\hline$\# 40390 \mathrm{~A}$ & 372 & & 1553 & & \\
\hline$\# 40390 \mathrm{~B}$ & 135 & & 3486 & & \\
\hline$\# 40590 \mathrm{~A}$ & 335 & & 2241 & & \\
\hline$\# 40590 \mathrm{~B}$ & 135 & & 2490 & & \\
\hline
\end{tabular}




\begin{tabular}{|c|c|c|c|c|c|}
\hline CSI\# & $\begin{array}{c}\text { Thickness } \\
(\mu \mathrm{m})\end{array}$ & $\begin{array}{c}\text { yield w/o Al } \\
(\mathrm{e} / \mathrm{MeV})\end{array}$ & $\begin{array}{l}\text { yield w/Al } \\
\text { (e/MeV) }\end{array}$ & $\begin{array}{l}\text { Evp.rate } \\
(\mu \mathrm{m} / \mathrm{min})\end{array}$ & $T /(m / 0)$ \\
\hline$\# 40690 \mathrm{~A}$ & 257 & & 4229 & & \\
\hline$\# 40690 \mathrm{~B}$ & 101 & & 5472 & & \\
\hline$\# 41890 \mathrm{~A}$ & 312 & & 3276 & 15.6 & \\
\hline$\# 41890 \mathrm{~B}$ & 104 & & 4762 & & \\
\hline$\# 42490 \mathrm{~A}$ & $2 \sqrt{33}$ & 12856 & 19686 & 6.7 & \\
\hline$\# 42490 \mathrm{~B}$ & 100 & 14045 & 20734 & & \\
\hline$\# 42590 \mathrm{~A}$ & 319 & 5326 & 7101 & 9.1 & \\
\hline$\# 42590 \mathrm{~B}$ & 137 & 8080 & 10774 & & \\
\hline \#50190A & 302 & 5064 & 7958 & 8.6 & \\
\hline$\# 50190 \mathrm{~B}$ & 132 & 7725 & 10484 & & \\
\hline$\# 50290 \mathrm{~A}$ & 461 & 4689 & 6630 & & \\
\hline$\# 50290 \mathrm{~B}$ & 191 & 7532 & 9304 & & \\
\hline$\# 50390 \mathrm{~A}$ & 301 & 5432 & 7605 & 7.52 & \\
\hline \#50390B & 126 & 9105 & 11950 & & \\
\hline$\# 50490 \mathrm{~A}$ & 275 & 17982 & 27723 & 4.583 & \\
\hline$\# 50490 \mathrm{~B}$ & 132 & 17665 & 27050 & & 0.032 \\
\hline$\# 50790 \mathrm{~A}$ & 238 & 17908 & 29052 & 2.311 & \\
\hline$\# 50790 \mathrm{~B}$ & 88 & 17652 & 29421 & & \\
\hline$\# 50890 \mathrm{~A}$ & 315 & 18550 & 29965 & 3.28 & 0.019 \\
\hline$\# 50890 \mathrm{~B}$ & 125 & 20030 & 30332 & & \\
\hline$\# 52490 A$ & 461 & 16825 & 27180 & 3.5 & \\
\hline$\# 52490 \mathrm{~B}$ & 194 & 16264 & 23297 & & \\
\hline$\# 60190 \AA$ & 317 & & & 3.52 & \\
\hline$\# 60190 \mathrm{~B}$ & 122 & 12796 & 19776 & & \\
\hline$\# 60690 \mathrm{~A}$ & 318 & 15290 & 23469 & 3.35 & 0.010 \\
\hline \#60690B & 139 & 14415 & 20288 & & \\
\hline \#61190i & 463 & 10668 & 15841 & 4.873 & 0.004 \\
\hline \#61190B & 179 & 11458 & 16042 & & \\
\hline \#61890A & 308 & 16534 & 24801 & 3.28 & \\
\hline \#61890B & 124 & 16109 & 21863 & & \\
\hline$\# 72390 \mathrm{~A}$ & 425 & & 27992 & 4.0 & 0.089 \\
\hline$\# 72390 \mathrm{~B}$ & 177 & & 28760 & & \\
\hline$\# 81790 \mathrm{~A}$ & 894 & 11449 & 18989 & 4.0 & \\
\hline$\# 81790 \mathrm{~B}$ & 340 & 11151 & 15931 & & \\
\hline$\# 82290 \mathrm{~A}$ & 1001 & & 20342 & 4.16 & \\
\hline 7"782290B & 452 & & 14556 & & \\
\hline$\# 90690 \bar{A}$ & 1027 & & 17828 & & \\
\hline \#90690B & 362 & & 16904 & & \\
\hline
\end{tabular}




\section{References}

1. I. Holl, E. Lorenz and G. Mageras, "A measurement of the Light Yield of Some Common Inorganic Scintillators," IEEE Trans. Nucl. Sci. NS-35 (1988)105.

2. Harshaw Radiation Detector, 1984, Harshaw, 6801 Cochran Road, Solon, Ohio 44139.

3. R. Ruchti, B. Baumbaugh, J. Bishop, N. Biswas, N. Cason, R. Erichsen, V. Kenney, A. Kreymer, R. Mountain and W. Shephard, "Scintillating Glass, Fiber-Optic Plate Detectors for Active Target and Tracking Applications in High Energy Physics Experiments," IEEE Trans. Nucl. Sci. NS-31 (1984)69.

4. Synergistic Detector Designs, 2438 Wyandotte Street, Building A, Mountainview, CA. 94043.

Collimated Hole. Inc., 460 Division Strt, Campbell, CA. 95008.

5. C.W. Bates, "Scintillation Processes in Thin Films of CsI(Na) and CsI(Tl) due to Low Energy X-rays, Electrons and Protons," Adv. Electronics and Electron Physics, 28A (1968) 451 .

6. A.L.N. Stevels and A.D.M. Schrama de Pauw, "Vapor-Deposited CsI:Na Layers, I. Morphologic and Crystallographic Properties, II Screens for Application in X-ray Imaging Devices," Philips Res. Repts 29(1974)340.

7. M. Tsuda and Y. Kimura, "Background Generation at Input Screens of X-ray Image Intensifiers," Jpn. J, Appl. Phys. Vol.24 (1985)1087.

8. S. Kubota, S. Sakuragi, S. Hashimoto and J. Ruan, "A New Scintillation Material: Pure CsI with 10ns Decay Time," Nucl. Instr. and Meth. A268(1988)275.

9. Photodiode catalogue March,1986, Hamamatsu Photonics K.K., 314-5 Shimokanzo, Toyooka-mura, Iwata-gun, Shizuoka-ken, 438-01, Japan.

10. H. Ito, S. Matsubara, Г. Takahashi, T. Shimada and H. Takeuchi, "Integrated Radiation Detectors with a-Si Photodiodes on Ceramic Scintillators," Jpn. J. Appl. Phys. Vol.28 (1989)L1476.

11. P. Schotanus, R. Kamermans and P. Dorebos, "Scintillation Characteristics of Pure and Tl-doped CsI Crystals," IEEE Trans. Nucl. Sci, NS-37 (1990)177.

12. R.D. Giauque, F.S. Goulding, J.M. Jaklevic and R.H. Pehl, "Trace Element Determination with Semiconductor X-ray Spectrometers, " Analytical Chemistry, Vol.45 (1973)671.

13. H. Ito, S. Matsubara, T. Takahashi, T. Shimada and H. Takeuchi, "Integrated Radiation Detectors with a-Si Photodiodes on Ceramic Scintillators," Jpn. J. Appl. Phys. Vol.28 (1989)L1476.

i4. L.E. Antonuk, J. Yorkston, J. Boudry, M.J. Longo, J. Jimenez and R.A. Street, "Development of Hydrogenated Amorphous Silicon Sensors for High 
Energy Photon Radiotherapy Imaging," IEEE Trans. Nucl. Sci, NS-37 (1990)165.

15. R.A.Street, S. Nelson, L. Antonuk and V.Perez-Mendez, "Amorphous Silicon Sensor Arrays for Radiation Imaging," Proc. Mater. Soc.Vol.192 (1990)441.

16. M. Kunii, K. Hasegawa, H. Oka, Y. Nakazawa, T. Takeshita and H. Kurihara, "Performance of a High-Resolution Contact-Type Linear Image Sensor with aSi:H/a-SiC:H Heterojunction Photodiodes," IEEE trans. Electron Devices, ED36 (1989)2877.

17. M. Kobayashi, S. Sakuragi, "Radiation damage of CsI(TI) crystals above $10^{3}$ rad," Nucl. Instr. Meth., A254 (1987)275.

18. M.J. Berger, S.M. Seltzer, S.E. Chappell, J.C. Humphreys and J.W. Mots, "Response of Silicon Detectors to Monoenergetic Electrons with Energies between 0.15 and 5.0 MeV," Nucl. Instr. and Meth. 69(1969)181.

19. T. Takahashi, H. Itoh, T. Shimada and H. Takeuchi, "Design of Integrated Radiation Detectors with a-Si Photodiodes on Ceramic Scintillators for use on XRay Computed Tomography," IEEE Trans. Nucl. Sci, NS-37 (1990) 1478.

20. K. Itoyama, "Properties of Sn-Doped Indium Oxide Prepared by High Rate and Low Temperature RF Sputtering," J. Joun. of Appl. Phys., Vol. 17 (1978)1191.

21. R.L. Weisfield and C.C. Tsai, "The Role of Carbon in Amorphous Silicon NIP Photodiode Sensors", Proc. Mater. Soc.Vol.192 (1990)423. 


\section{Chapter_5._Apolications_of_a-SiiH_radiation detectors}

It has been shown that a-Si:H is capable of detecting radiation either directly in a thick diode or indirectly in a phosphor material coupled to a thin photodiode. Although this is sufficient as a radiation monitor, a wider range of application will require addition of position sensitivity to a-Si:H radiation detectors. Schemes to achieve position sensitivity and some expected application fields of radiation imaging devices are discussed in this chapter.

\section{5-1. Position sensitivity}

One dimensional (ID) or two dimensional (2D) position sensitivity may be achieved by dividing the electrode into narrow strips or small pixels and providing individual readout electronics for each strip or pixel. This is shown schematically in Fig.5-1. Onedimensional readout electronics for single-particle detection is available in the form of crystalline silicon integrated circuit chips such as the SVX chip designed at LBL for high energy physics experiments [1]. The top electrode of an a-Si:H diode is divided into narrow strips and each strip is wire-bonded to the separate amplifier channel of these IC chips to take advantage of the existing crystalline Si electronics. Single-particle-counting surip detectors can be built this way as shown in Fig.1-5 (a). A strip or pixel device with the front end electronics made from a-Si:H or poly-Si TFTs on each strip or pixel as shown in Fig.1-5 (b) and (c) has been proposed [2,3]. For fluence detection, the readout electronics could be simple switching devices to address strips sequentially as in the case of facsimile heads. For individual particle or single $\gamma$ photon counting, the readout electronics must contain a high-gain charge-sensitive amplifier stage and a sequential addressing stage as is contained in the SVX chip [1]. As shown in Fig.5-1 (c), vertical integration of the electronics and the addressing bus lines would enable readout of each pixel signal without reducing the sensitive area to radiation. Also shown is a schematic of the electronics on each pixel. Again, fluence detection needs a simple switching device to 
address $\mathrm{X}$ and $\mathrm{Y}$ coordinates while single particle counting requires a charge-sensitive amplifier stage in addition to the addressing stage. 


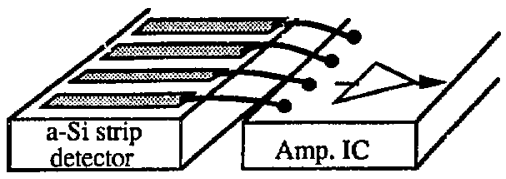

(a)

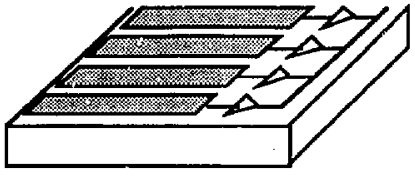

(b)

PIXEL
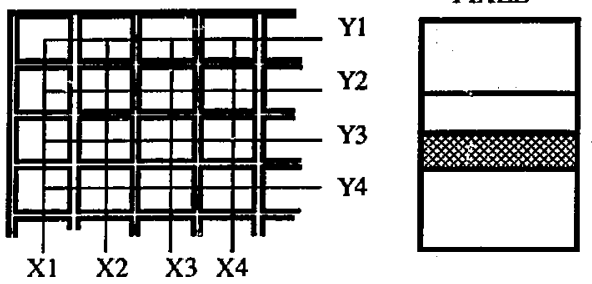

Detector

Readout Bus
Electronics

Substrate

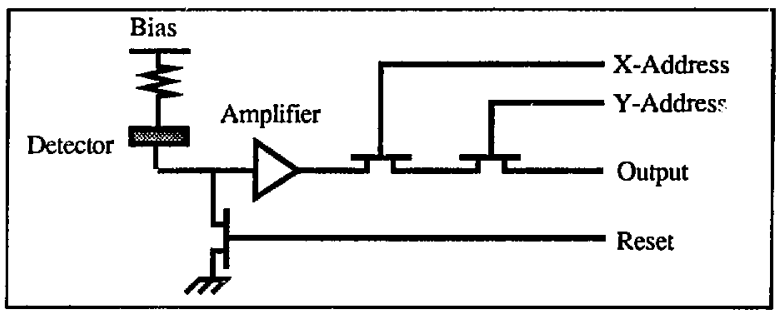

(c)

Fig.5-1 Schematics of position sensitive detectors (PSDs).

(a) One dimensional PSD (strip detector).

(b) One dimensional PSD with the built-in readout electronics made of a-Si:H or poly-Si thin film transistors (TFTs).

(c) Two dimensional PSD (pixel detector) with the built-in readout electronics made of a-Si:H or poly-Si TFTs.

\section{5-2. High energy physics applications}

A number of secondary charged particles are emitted following a collision event of primary particles in high energy physics experiments. These particles can be detected by 
position sensitive a- $\mathrm{Si}: \mathrm{H}$ radiation detectors surrounding the collision site as snown schematically in Fig.5-2. Tracks of each particle can be identified by processing information from each detector. Thick a-Si:H layers formed into small pixels are essential to provide high signal to noise $(S / N)$ ratio for direct detection of these particles. With small pixel detectors, noise contributions from the detector capacitance, leakage current and the carrier trapping and emission in a-Si:H layer are expected to be low enough to provide $\mathrm{S} / \mathrm{N}$ ratio high enough for direct detection of the minimum ionization events. Due to the large area and high radiation resistivity required for this tracking device, a-Si:H radiation detectors may have advantages over the crystalline devices currently under development [4].

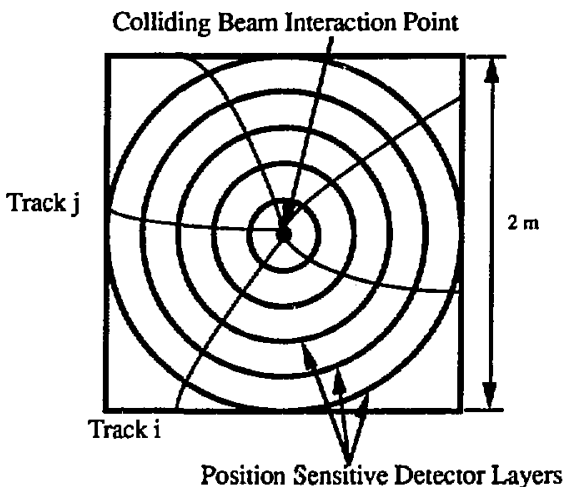

Fig.5-2 Tracking device. Particle tracks are identified by processing the information from the PSDs surrounding the colliding beam interaction point.

\section{5-3. Medical imaging}

There are marly radiation detection devices used in medical imaging ranging from simple ones such as X-ray film, to elaborate systems such as a Positron Emission Tomograph (PET). We discuss the use of a-Si:H detectors in medical imaging by dividing these 
existing devices into two groups i.e. flux imaging devices and single fhoton imaging devices.

\section{5-3-1. Flux imaging devices}

$\mathrm{X}$-ray film has been widely used due to its excellent spatial resolution despite the limited dynamic range, non-linear response, lack of real-time capability, inability for repeated use and need for additional efforts to obtain digitized images. The imaging plate developed by Fuji [5] solves most of the deficiencies of film except for the real-time capability. It requires a separate readout process to "develop" an image. An X-ray image intensifier is a real-time device and is often used in X-ray digital imaging [6]. The detector system used for digital radiology consists of an image intensifier and a TV camera for image digitization. Since an image intensifier is a vacuum tube, it is bulky and mechanically delicate.

A pixel detector combined with a scintillation faceplate as shown in Fig.5-3 could be used in place of these devices. The TFT front-end electronics as shown schematically in Fig.5-4 read out charges stored in each pixel. A simple calcuiation below shows that the expected signal size is large enough to simplify the requirements for the readout electronics. Consider a typical X-ray exposure of 0.1 Roentgen as normally used for chest $\mathrm{X}$-ray imaging. This corresponds to approximately $3 \times 10^{+9} 50 \mathrm{keV} \mathrm{X}$-ray photons $/ \mathrm{cm}^{2}$. About $80 \%$ of these $\mathrm{X}$-ray photons would be absorbed by a $300 \mu$ m-thick CsI(TI) layer. From

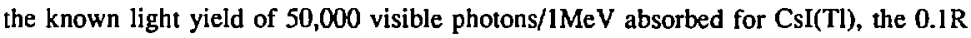
exposure produces $6 \times 10^{+12}$ photons $/ \mathrm{cm}^{2}$ in the $\mathrm{CsI}(\mathrm{Tl})$. As a conservative estimate, assume $50 \%$ light collection and $60 \%$ light to electron-hole pair conversion efficiency (quantum efficiency) of the a-Si:H photodiode. If we have $10^{+4}$ pixels $/ \mathrm{cm}^{2}(100 \mu \mathrm{m} \mathrm{x}$ $100 \mu \mathrm{m}$ pixel size), each pixel produces $1.8 \times 10^{+8}$ electron-hole pairs. The capacitance of a $1 \mu \mathrm{m}$ thick $100 \mu \mathrm{m} \times 100 \mu \mathrm{m}$ pixel is about $1 \mathrm{pF}$. Therefore, the average voltage signal on each pixel after $0.1 \mathrm{R}$ exposure is about $30 \mathrm{~V}$. The spatial resolution is determined by the pixel size and the scintillation light spread in the phosphor. The output image is 
inherently digitized and well-suited for sophisticated digital image processing techniques. The dynamic range required for these applications is about 1000 . Whether these devices are capable of achieving this dynamic range depends on many design considerations and is beyond the intended scope of this chapter.

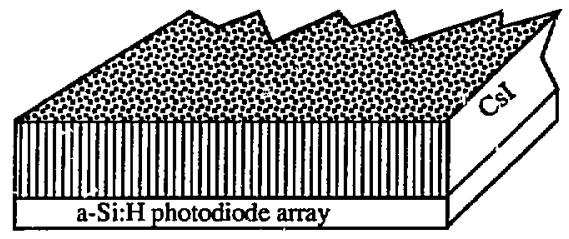

Fig.5-3 2D-PSD. A 2D a-Si:H photodiode array combined with either a $300 \mu \mathrm{m}$ Cs $\backslash(\mathrm{Tl})$ with fine columnar structure or a scintillation faceplate containing a rare-earth element can be used for recording $\mathrm{X}$-ray transmission images.

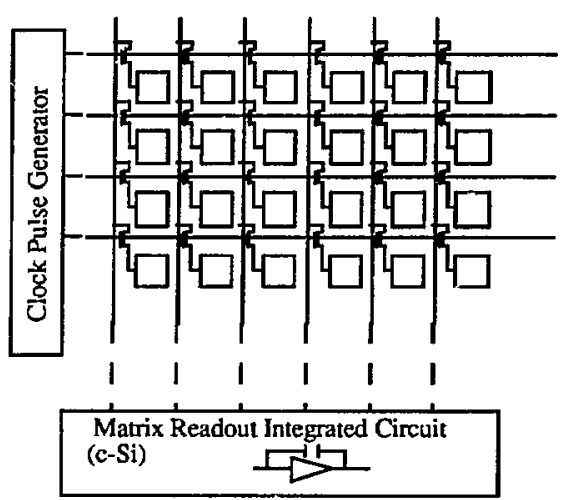

Fig.5-4 Readout electronics for the $2 \mathrm{D}$ array.

An X-ray Computed Tomograph (CT) is a more elaborate high flux imaging system [7], but the detector itself is not necessarily more sophisticated. A schematic drawing of an $X$ ray $\mathrm{CT}$ is shown in Fig.5-5. An X-ray-tube emitting a fan beam of X-rays is rotated around the body together with a one dimensional position sensitive X-ray detector (ID- 
PSD). The transmission data through the patient's body known as projection profiles are stored in the computer for each $\mathrm{X}$-ray-tube position and are used to reconstruct the transverse cross-sectional inage of the $\mathrm{X}$-ray attenuation coefficient distribution. An array of Xe-tilled ionization chambers is normally used as the 1D-PSD. Due to the geometrical magnification, the spatial resolution of this detector can be as low as a few $\mathrm{mm}$. A strip detector as shown in Fig.5-1(a),(b) combined vith a phosphor should work as well as the Xe chamber. Again, no elaborate readout amplifier is needed since it is a flux detector.

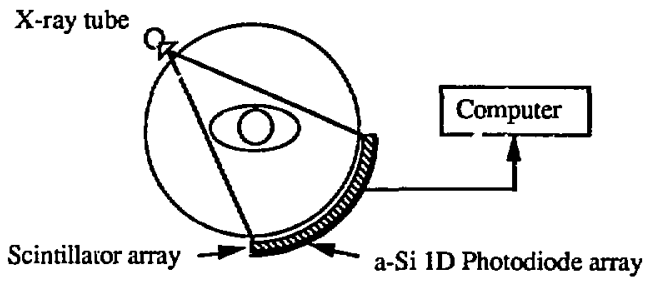

Fig.5-5 A 1D a-Si:H photodiode array combined with a similar 1D scintillator array can be used in place of the Xe-filled ionization chamber currently employed in an X-ray CT.

\section{5-3-2. Single photon imaging devices}

In nuclear medicine, radiopharmaceuticals are introduced into the body and $\gamma$ rays emitted from the body are detected to give an image of the radionuclide distribution Here, it is necessary to count individual $\gamma$ rays, unlike the fux detectors where the charges created by a number of $\mathrm{X}$-rays are accumulated during the duration of the $\mathrm{X}$-ray pulse. The scintillation camera is an example of this type of imaging device. It consists of a slab of $\mathrm{NaI}(\mathrm{Tl})$ coupled to a number of photomultiplier tubes (PMTs). Centroid calculation of the PMT outputs gives the position information of the incidert $y$ ray and the surn of all the PMT outputs gives the energy of the $\gamma$ ray. A mechanical collimator is placed in front of the scintillator to accept only the desired $\gamma$ rays to make an image of the radionuclide distribution in the body projected on the plane defined by the scintillator slab. A pixel 
device combined with a high light yield scintillator faceplate as shown schematically in Fig.5-6 can replace this scintillation camera. Here, the scintillator must be carefully chosen and CsI(Tl) appears promising because of its high light yield, high atomic number and its built-in columnar structure. The thickness of CsI(T1) should be more than $5 \mathrm{~mm}$ to ensure good detection efficiency for the most-commonly used $140 \mathrm{keV} \gamma$ rays from the Tc$99 \mathrm{~m}$ compounds. The intrinsic spatial resolution of conventional scintillation cameras is more than $3 \mathrm{~mm}$. A pixel device shown in Fig.5-1 can have much higher spatial resolution if required. Since it doesn't have PMTs, it is compact, light weight, and wotld nced less routine adjustments than the conventional PMT array.

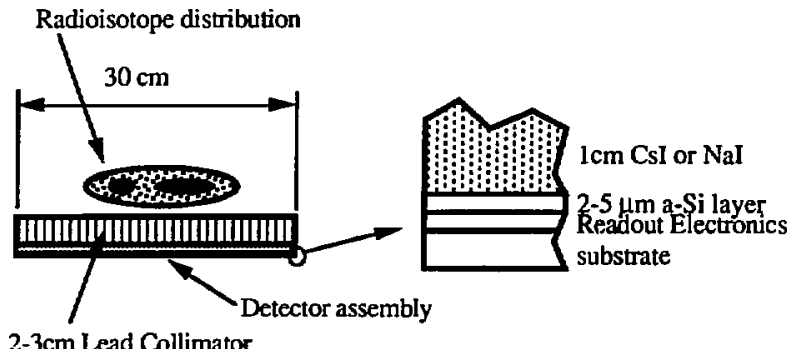

$2-3 \mathrm{~cm}$ Lead Collimator

Fig.5-6 Scintillation camera

\section{5-4. Material science and life science applications}

Radiation detectors are used in material science for recording diffraction patterns in $\mathrm{X}$ ray crystallography and $X$-ray or synchrotron radiation transmission images in nondestructive studies of materials. Bateman gives a review of the existing devices used for these studies [8]. As pointed out there, the principal restriction on the wide-spread use of seriniconductor detectors is their limited area. A-Si:H may be a better choice for the detector material for such applications. 
In X-ray crystallography, monoenergetic characteristic X-rays such as the $\mathrm{Cu} \alpha$-line (8 keV) are detected by curved or flat 1D-PSD such as film or multiwise proportional chambers(MWPC) $[9,10]$. A-Si:H alloys on flexible substrates can be used in place of film or MWPC detectors. Since the diffracted X-ray flux intensity is low for these studies, single photon detection is preferred over flux integration. The pulse mode operation (counting a single $\mathrm{X}$-ray photon) of the indirect detection scheme is somewhat difficult because, for example, one $8 \mathrm{keV} \mathrm{X-ray} \mathrm{photon} \mathrm{creates} \mathrm{only} \mathrm{about} 400$ photons in CsI(Tl). Direct detection of a single $8 \mathrm{keV} \mathrm{X}$-ray photon needs careful design of the readout electronics because the expected number of electron-hole pairs is around 1300. Table 5-1 summarizes the probability of photoelectric effect of $8 \mathrm{keV}$ and $17 \mathrm{keV} \mathrm{X-ray} \mathrm{photons} \mathrm{in}$ three kinds of a-Si:H alloys i.e. $100 \% \mathrm{Si}, 90 \% \mathrm{Si}+10 \% \mathrm{Ge}, 80 \% \mathrm{Si}+20 \% \mathrm{Ge}$. ASi:Ge:H alloys with good quality can be made with the Ge content up to $20 \%$. Layer thicknesses of 30-50 $\mu \mathrm{m}$ could provide similar detection efficiency as the pressurized Xefilled MWPCs.

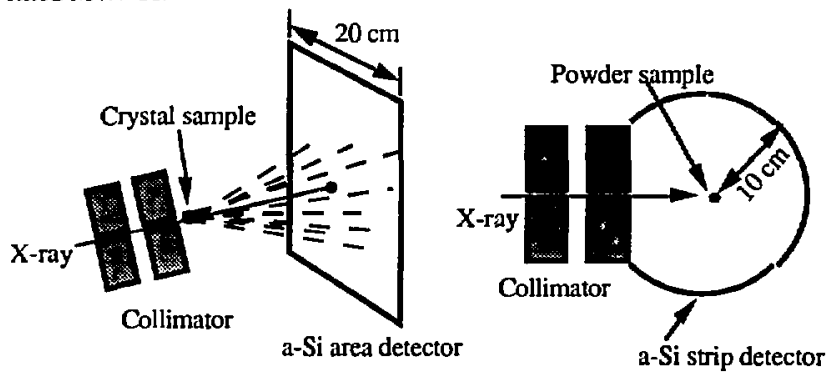

Fig.5-7 X-ray deflection experiment applications

Table 5-1. Detection efficiency of a-Si:Ge:H alloys

\begin{tabular}{|c|c|c|c|c|c|c|}
\hline & \multicolumn{2}{|c|}{$100 \% \mathrm{Si}$} & \multicolumn{2}{c|}{$90 \% \mathrm{Si}+10 \% \mathrm{Ge}$} & \multicolumn{2}{c|}{$80 \% \mathrm{Si}+20 \% \mathrm{Ge}$} \\
\hline X-ray energy & $30 \mu \mathrm{m}$ & $50 \mu \mathrm{m}$ & $30 \mu \mathrm{m}$ & $50 \mu \mathrm{m}$ & $30 \mu \mathrm{m}$ & $50 \mu \mathrm{m}$ \\
\hline $8 \mathrm{keV}$ & 0.37 & 0.53 & 0.45 & 0.63 & 0.53 & 0.72 \\
\hline $17 \mathrm{keV}$ & 0.051 & 0.083 & 0.18 & 0.28 & 0.31 & 0.46 \\
\hline
\end{tabular}


In non-destructive studies of materials, where a flux of higher energy $\mathrm{X}$-rays is employed, either direct or indirect detection of these $\mathrm{X}$-rays is possible by the a-Si:H detectors operated by the integral mode (flux counting).

Even for X-ray microscopy [11] where the size advantage of a-Si:H is less significant, its high radiation resistivity is a desirable feature for these devices. When a large geometrical magnification is employed by making the ratio of the $\mathrm{X}$-ray source-object distance to the object-detector distance small, a large detector area such as $10 \times 10 \mathrm{~cm}^{2}$ becomes important again.

In biology, autoradiography is a powerful tool for obtaining information on protein distributions and DNA sequences from radioisotope-labellex plates of organic bodies and electrophoresis gels. Radioisotopes commonly used for these studies are $\mathrm{H}-3, \mathrm{C}-14$ and P32. These are all $\beta$-emitters whose maximum energies are $18 \mathrm{keV}, 155 \mathrm{keV}$ and $1.7 \mathrm{MeV}$, respectively. Positions and intensity distributions of these $\beta$-emitters are found by detecting the $\beta$ particles escaping from the plate or the gel with a $1 \mathrm{D}$, or more recenily $2 \mathrm{D}$ PSD in clese contact. X-ray film has been used widely. Multiwire proportional chambers (MWPC) have been applied for ID or 2D radiochromatography [12]. To ensure sufficient sensitivity, the thickness of the gas-filled detector is about $1 \mathrm{~cm}$, causing spatial resolution degradation due to the parallax error. Single $\beta$ particle detection for these radioisotopes can be done by a- $\mathrm{Si}: \mathrm{H}$ radiation detectors either directly or indirectly with the exception of $\mathrm{H}-3$. The detector thickness can be less than I mm and the sensitive area can be placed very close to the plates or gels. H.3 detection requires careful design considerations on the readout electronics due to the small signal size. 


\section{5-5. Conclusion}

Some expected application areas of a-Si:H radiation detectors were discussed. Although the basic possibilities of detecting radiation with a-Si:H layers have been demonstrated in previous chapters, extensive further design and development considerations will be required for a prototype fabrication for each of these application field described in this chapter. Design considerations for the readout electronics for single particle detection and noise measurements of the a-Si:H and poly-Si TFTs are under way and some results have been published [2,3]. While this electronics development needs a fair amount of effort, such effort could be shared with industry which should find a large market for a-Si:H radiation detector applications such as described in this chapter. 


\section{References}

1. S.A.Kleinfelder, W.C.Carithers, R.P.Ely, C.Haber, F.Kirsten and H.G.Spieler, "A Flexible 128 Channel Silicon Strip Detector Instrumentation Integrated Circuit with Sparse Data Readout," IEEE Trans. Nucl. Sci. NS-35(1988)171.

2. V.Percz-Mendez, G.Cho, I.Fujieda, S.N.Kaplan, S.Qureshi and R.A. Street, "Proposed Thin Film Electronics for a-Si:H PIXEL Detectors," LBL-26254.

3. G. Cho, M. Conti, J. S. Drewery, I. Fujieda, S. N. Kaplan, V. Perez-Mendez, S. Qureshi and R. A. Street, "Assessment of TFT Amplifiers for a-Si:H PIXEL Particle Detectors", LBL-27860, IEEE Trans. Nuc. Sci. NS-37(1996)1 142.

4. E.H.M. Heijne and P. Jarron, "Development of Silicon Pixel Detectors: an Introduction," Nucl. Instr. and Meth. A275 (1989) 467.

5. J. Miyahara, K. Takahashi, Y. Amemiya and N. Kamiya, "A New Type of X-Ray Area Detector Utilizing Laser Stimulated Luminescence," Nucl. Instr. and Meth. A246 (1986)572.

6. H. Roehrig, S. Nudelman, H.D. Fisher, M.M. Frost and M.P. Capp, "Photoulectronic Imaging for Radiology," IEEE Trans. Nucl. Sci. NS-28 (1981)190.

7. L. Axel, P.H. Arger and R.A. Zimmerman, "Applications of Computerized Tomography to Diagrostic Radiology," Proc. IEEE Vol.71 No.3 (1983)293.

8. J.E. Bateman, "Detectors for Condensed Matter Studies," Nucl. Instr. and Meth. A273 (1988) 721.

9. V. Perez-Mendez, P. Wiedenbeck, C.N. Wagner and E. Woelfel, "Curved Anode Wire Chambers for X-ray Diffraction Applications," Nucl. Instr. and Meth. $228(1985) 564$.

10. Hamlin, Cork, Howard, Nielsen, Vernon, Matthews, Perez-Mendez, Xuong, "Characteristics of Flat Multiwire Area Detectors for Protein Crystallography," J. Appl. Crystallography 14(1981)85.

11. J.E. Bateman, G.R. Moss, P. Rocket and S Webb, "Digital X-Ray Microscopy with a Microfocal X-Ray Generator and an MWPC Area Detector," Nucl. Instr. and Meth. A273 (1988)767.

12. C.W.E. Van Eijk, R.W. Hollander and S.M. Van der Sluis, "An MWPC for Beta Radiochromatography," Nucl. Instr. and Meth. A273 (1988)764. 


\section{Chapter 6. Conclusion}

Hydrogenated amorphous silicon (a-Si:H) has been investigated as a material for radiation detectors.

Charge collection and signal generation process are analyzed in terms of carrier mobilities, lifetimes and electric field. Charge collection in thick a-Si:H diodes is often limited by deep-level trapping of carriers during transit and a finite charge integration time required for single particle counting in some applications and sometimes by volume recombination of carriers for detecting heavily-ionizing particles such as $\alpha$ particles. The charge collection process is also strongly affected by the non-uniform electric field profiles in a-Si:H diodes caused by the fixed space charges inside the material under reverse-bias. Signal generation due to a week light pulse irradiating each end of a thick diode is measured as a function of a reverse-bias and it gives a valuable information about the fixed space charges.

Field profiles in a-Si:H radiation detectors can be manipulated by either doping, electrode geometry, or a combination of both. The buried $p$-i-n structure improves the charge collection process by allowing one to apply higher bias while suppressing the nearsurface breakdown phenomenon. It also gives a bias safety margin before breakdown for each element of a large area detector. Making the $p$ layer of the conventional $p-i-n$ diode thicker also enables high bias operation. Some other ideas to shape the field profile and their calculation results show that thicker a-Si:H layers may become fully-depleted with a non-uniform field profile. The microstrip electrode provides a similar charge collection efficiency to the parallel plate configuration with a smaller capacitance.

$\mathrm{X}$-ray detection with a good sensitivity is demonstrated by an a-Si:H photodiode coupled to an evaporated CsI scintillator. The scintillation quality of evaporated CsI layers can be made almost identical to its single crystal counterpart. 
These a-Si:H radiation detectors will find their applications in high energy physics experiments, medical imaging, material science and life science. Each application imposes different requirements on the radiation detector systems. Whether a-Si:H detectors can replace existing devices such as film, gas-filled detectors, scintillation detectors and crystalline semiconductor detectors strongly depends on each application and it must be judged case by case. However in general, the following advantages of the a-Si:H detectors over the existing devices are expected:

(a) larger area, higher radiation resistance and lower cost than crystalline semiconductor detectors.

(b) higher spatial resolution and detection efficiency than gas-filled detectors.

(c) they are better suited for medical digital radiography than film or image intensifiers. 